\title{
Analysis of chemical abundances in planetary nebulae with [WC] central stars
}

\section{Chemical abundances and the abundance discrepancy factor ${ }^{\star}$}

\author{
Jorge García-Rojas ${ }^{1,2}$, Miriam Peña ${ }^{3}$, Christophe Morisset ${ }^{1,3}$, Gloria Delgado-Inglada ${ }^{3}$, \\ Adal Mesa-Delgado ${ }^{4}$, and María Teresa Ruiz ${ }^{5}$
}

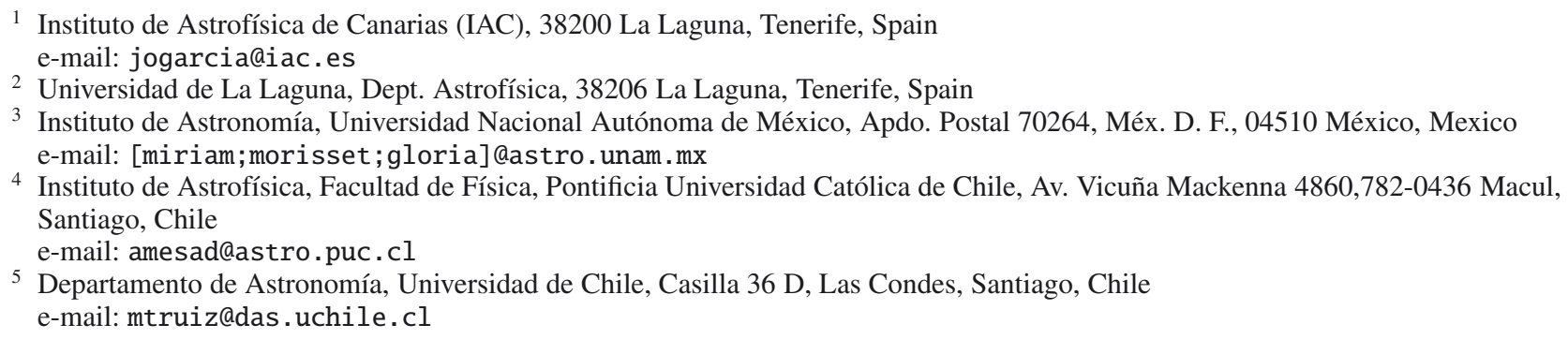

Received 24 July 2013 / Accepted 6 September 2013

\begin{abstract}
Aims. We present the abundance analysis of 12 planetary nebulae ionized by [WC]-type stars and weak-emission-line stars (wels) obtained from high-resolution spectrophotometric data. Our main aims are to determine the chemical composition of the nebulae and to study the behaviour of the abundance discrepancy problem (ADF) in this type of planetary nebulae.

Methods. The detection of a large number of optical recombination lines (ORLs) and collisionally excited lines (CELs) from different ions $\left(\mathrm{O}^{+}, \mathrm{O}^{++}, \mathrm{C}^{++}, \mathrm{C}^{+3}\right.$ and $\left.\mathrm{Ne}^{++}\right)$were presented previously. Most of the ORLs were reported for the first time in these PNe, which increased the sample of PNe with detected faint ORLs. Ionic abundances were determined from the available CELs and ORLs, using previously determined physical conditions. Based on these two sets of ionic abundances, we derived the total chemical abundances in the nebulae using suitable ionization correction factors (when available).

Results. In spite of the [WC] nature of the central stars, moderate $\operatorname{ADF}\left(\mathrm{O}^{++}\right)$in the range from 1.2 to 4 were found for all the objects. We found that when the quality of the spectra is high enough, the ORLs $\mathrm{O}^{++} / \mathrm{H}^{+}$abundance ratios obtained from different multiplets excited mainly by recombination are very similar. Possible dependence of ADFs on some nebular characteristics such as surface brightness and nebular diameter were analysed, but we found no correlation. Abundances derived from CELs were corrected by determining the $t^{2}$ temperature fluctuation parameter. $\mathrm{O}$ abundances for PNe, derived from ORLs, are in general higher than the solar abundance. We derived the C/O ratio from ORLs and N/O and $\alpha$-element/O ratios from CELs and found that these PNe are, on average, richer in $\mathrm{N}$ and $\mathrm{C}$ than the average of the large PN samples. About half of our sample is $\mathrm{C}$-rich $(\mathrm{C} / \mathrm{O}>1)$. The growth of $\alpha$-elements is correlated with the $\mathrm{O}$ abundance. Comparing the $\mathrm{N} / \mathrm{O}$ and $\mathrm{C} / \mathrm{O}$ ratios with those derived from stellar evolution models, we estimate that about half of our PNe have progenitors with initial masses similar to or larger than $4 M_{\odot}$. No correlation was found between the stellar [WC] type and the nebular chemical abundances. A rough $\mathrm{O}$ abundance gradient computed for our limited PN sample, compared with the gradient obtained for $\mathrm{H}$ II regions, shows that there is a large dispersion in estimates of the $\mathrm{PNe} \mathrm{O}$ abundance for a given Galactocentric distance. The PN gradient is flatter than that for $\mathrm{H}$ II regions and at the solar distance and farther out, the PNe have a higher $\mathrm{O}$ abundance than $\mathrm{H}$ II regions, similarly to what is found in other spiral galaxies. This fact has no convincing explanation so far.
\end{abstract}

Key words. stars: AGB and post-AGB - ISM: abundances - planetary nebulae: general - Galaxy: abundances

\section{Introduction}

Planetary nebulae $(\mathrm{PNe})$ are produced at the final stages of low to intermediate-mass stars (LIMS, initial masses between 1 and $8 M_{\odot}$ and ages from 0.1 to $9 \mathrm{Gyr}$ ) by ejection of the outer stellar atmosphere shells. Thus, the chemical composition of $\mathrm{PNe}$ contains elements present in the interstellar medium (ISM) at the time of the star formation, and elements processed in the stellar nucleus that are dredged-up to the stellar surface at different moments of the stellar evolution. Of the central stars

\footnotetext{
^ Based on data obtained at Las Campanas Observatory, Carnegie Institution.
}

of PNe, about $10 \%$ belong to the [WC] class. These stars are $\mathrm{H}$-deficient, their atmospheres are $\mathrm{He}, \mathrm{C}$, and O-rich and they present intense winds, with terminal velocities ranging from several hundred to several thousand $\mathrm{km} \mathrm{s}^{-1}$ and mass loss rates of about $10^{-5}-10^{-6} M_{\odot} \mathrm{yr}^{-1}$ (see e.g., Koesterke 2001, and references therein). The surrounding PNe, on the other hand, show a normal level of $\mathrm{H}$ (H-rich gas).

Owing to their H-deficient composition, [WC] central stars are helium-burning objects. It has been found that [WC] central stars are mainly distributed in [WC]-late types ([WC8] to [WC11]) with stellar temperatures in the range from $30 \mathrm{kK}$ to about $70 \mathrm{kK}$, that are surrounded by young and dense nebulae, 
Table 1. $\mathrm{He}^{+}$and $\mathrm{He}^{++}$abundances ${ }^{a}$.

\begin{tabular}{|c|c|c|c|c|c|c|}
\hline & Cn 1-5 & $\mathrm{Hb} 4$ & He 2-86 & M 1-25 & M 1-30 & M 1-32 \\
\hline Lines & 12 & 11 & 12 & 16 & 16 & 13 \\
\hline$\tau_{3889}$ & $6.23 \pm 1.09$ & $13.51 \pm 2.78$ & $17.67 \pm 1.62$ & $13.22 \pm 2.04$ & 12.93: & $5.51 \pm 2.35$ \\
\hline & 6.9 & 10.2 & 33.4 & 9.3 & 15.3 & 10.5 \\
\hline $\mathrm{He}^{+} / \mathrm{H}^{+b}$ & $1380 \pm 28$ & $934 \pm 43$ & $1226 \pm 24$ & $1236 \pm 35$ & $1306 \pm 35$ & $1257 \pm 47$ \\
\hline \multirow[t]{2}{*}{$\mathrm{He}^{++} / \mathrm{H}^{+}$} & - & $203 \pm 6$ & - & $0.39 \pm 0.16$ & - & $3.75 \pm 0.44$ \\
\hline & M 1-61 & M3-15 & NGC 5189 & NGC 6369 & PC 14 & Pe 1-1 \\
\hline Lines & 16 & 12 & 13 & 14 & 13 & 14 \\
\hline$\tau_{3889}$ & $16.50 \pm 1.87$ & $14.21 \pm 2.28$ & $3.20 \pm 0.72$ & $8.48 \pm 1.83$ & $5.88 \pm 1.41$ & $10.50 \pm 1.67$ \\
\hline & 28.3 & 17.0 & 7.9 & 9.3 & 6.0 & 7.3 \\
\hline $\mathrm{He}^{+} / \mathrm{H}^{+b}$ & $1086 \pm 32$ & $1055 \pm 35$ & $847 \pm 21$ & $1033 \pm 32$ & $1036 \pm 31$ & $1054 \pm 34$ \\
\hline $\mathrm{He}^{++} / \mathrm{H}^{+}$ & - & - & $376 \pm 6$ & $3.96 \pm 0.36$ & $41.5 \pm 1.2$ & $0.47 \pm 0.19$ \\
\hline
\end{tabular}

Notes. ${ }^{(a)}$ In units of $10^{-4}$. Errors correspond to the uncertainties in line flux measurements. ${ }^{(b)}$ It includes all uncertainties related to line intensities, $n_{\mathrm{e}}, \tau_{3889}$, and $t^{2}$.

and [WC]-early types ([WC4] to [WO2]), with stellar temperatures higher than $100 \mathrm{kK}$ that are surrounded by evolved nebulae. Thus there is no defined evolutionary status for these stars, and they evolve from the asymptotic giant branch (AGB) phase as do the other PN nuclei. Three different scenarios have been proposed for the formation of a [WC] central star: a late thermal pulse (LTP), a very late thermal pulse (VLTP) and an asymptotic giant branch final thermal pulse (AFTP) (see Herwig 2001; Blöcker 2001, for a description of these scenarios). Regarding their distribution in the Galaxy, a number of Wolf-Rayet planetary nebulae (hereinafter WRPNe) have been discovered in the Galactic bulge (Górny et al. 2004), and recently it has been found that WRPNe in the disk belong to a thinner disk than the average of PNe, probably indicating that they are young objects (Peña et al. 2013).

A few years ago we started an analysis of the chemical composition of PNe around [WC] central stars (WRPNe), aiming to determine these abundances via collisionally excited lines (CELs) and optical recombination lines (ORLs) and to compare the two results. Three WRPNe were studied by García-Rojas et al. (2009). In the present work, we analyze the chemical abundances for a sample of 12 Galactic PNe with [WC] or weak-emission-lines (wels) central stars. In a previous paper (García-Rojas et al. 2012, hereafter Paper I) we have presented the high-resolution spectrophotometric data for these objects, obtained at Las Campanas Observatory (LCO, Carnegie Institution) with the $6.5 \mathrm{~m}$ Magellan telescope Clay and the double echelle Magellan Inamori Kyocera Spectrograph (MIKE). An analysis of the physical conditions (electron temperatures, $T_{\mathrm{e}}$, and electron densities, $n_{\mathrm{e}}$ ) for each object, along with a discussion of the diagnostics that better represent $T_{\mathrm{e}}$ and $n_{\mathrm{e}}$ for each nebulae, was presented there ${ }^{1}$. In the present paper we use the results of Paper I to determine ionic and total chemical abundances for He and several heavy ${ }^{2}$ elements. We have added two additional objects from García-Rojas et al. (2009) (PB 8 and NGC 2867). As in García-Rojas et al. (2009), the main focus of this work is on the abundance discrepancy problem, that is, the

\footnotetext{
1 In Paper I, the diagnostic diagram for M 1-61 in Fig. 7 shows an incorrect $T_{\mathrm{e}}([\mathrm{N} \mathrm{II}])$ due to a typo in the data file. The correct value is higher and agree with the value presented in their Table 4.

2 Although it is not strictly correct, it is a widely used convention in nebular astrophysics to refer to all elements heavier than $\mathrm{He}$ as heavy, and we followed this convention.
}

difference between the chemical composition of the photoionized plasma as derived from CELs and from ORLs. These differences are commonly found in photoionized nebulae and reach to a factor of 2-3 in H II regions (García-Rojas \& Esteban 2007) and up to 70 in PNe, with an averaged value of about 3 (McNabb et al. 2013). These discrepancies are parametrized through the abundance discrepancy factor (ADF) which is defined as

$$
\operatorname{ADF}\left(X^{+\mathrm{i}}\right)=\left(X^{+\mathrm{i}} / \mathrm{H}^{+}\right)_{\mathrm{ORLs}} /\left(X^{+\mathrm{i}} / \mathrm{H}^{+}\right)_{\mathrm{CELs}},
$$

where $X^{i+}$ is the $i+$ ionic abundance of an element $X$ and $\mathrm{H}^{+}$is the abundance of ionized hydrogen. Since one of the possible mechanisms proposed to cause high ADFs are tiny metal-rich inclusions embedded in the nebular plasma (Liu et al. 2006, and references therein), we consider that WRPNe are nebulae with high probability of having such inclusions, because the central stars are $\mathrm{H}$-deficient and present in their stellar winds $\mathrm{He}, \mathrm{C}$, and O-rich material.

In the following we present the ionic abundances (Sect. 2) for He and for heavy elements; for some of these elements, we have derived abundances from both CELs and ORLs. Total abundances and a discussion of the obtained results are presented in Sects. 3 and 4. In Sect. 5, the ADFs are presented and discussed. A final discussion is presented in Sect. 6. Our conclusions can be found in Sect. 7.

\section{Ionic chemical abundances}

\section{1. $\mathrm{He}^{+}$and $\mathrm{He}^{++}$abundances}

We have detected several He I emission lines in the spectra of each nebulae. These lines arise mainly from recombination, but some of them can be affected by collisional excitation and selfabsorption effects.

We used the effective recombination coefficients of Storey \& Hummer (1995) for H I and those computed by Porter et al. (2005), with the interpolation formulae provided by Porter et al. (2007) for He I. The collisional contribution was estimated from Sawey \& Berrington (1993) and Kingdon \& Ferland (1995), and the optical depth in the triplet lines was derived from the computations by Benjamin et al. (2002). We determined the $\mathrm{He}^{+} / \mathrm{H}^{+}$ratio from a maximum-likelihood method (MLM, Peimbert et al. 2000, 2002). To self-consistently determine $n_{\mathrm{e}}(\mathrm{He} \mathrm{I}), T_{\mathrm{e}}(\mathrm{He} \mathrm{I})$, $\mathrm{He}^{+} / \mathrm{H}^{+}$ratio and the optical depth in the He I $\lambda 3889$ line, $\left(\tau_{3889}\right)$, 
Table 2. Physical conditions for abundance determinations.

\begin{tabular}{ll}
\hline \hline Phys. cond & Ions \\
\hline$T_{\mathrm{e}}([\mathrm{N}$ II $]), n_{\mathrm{e}}(\text { low to medium })^{a}$ & $\mathrm{~N}^{+}, \mathrm{O}^{+}, \mathrm{S}^{+}, \mathrm{Cl}^{+}, \mathrm{Fe}^{+}$and $\mathrm{Fe}^{++}$ \\
$T_{\mathrm{e}}([\mathrm{O}$ III $]), n_{\mathrm{e}}(\text { low to medium })^{a}$ & $\mathrm{O}^{++}, \mathrm{S}^{++}, \mathrm{Cl}^{++}, \mathrm{Ar}^{++}$and $\mathrm{Fe}^{3}+$ \\
$T_{\mathrm{e}}([\text { Ar IV }])^{b}, n_{\mathrm{e}}(\text { high })^{a}$ & $\mathrm{Cl}^{+3}, \mathrm{Ar}^{+3}, \mathrm{Ar}^{+4}, \mathrm{Ne}^{++}, \mathrm{Ne}^{+3}$ and $\mathrm{Ne}^{+4}$ \\
\hline
\end{tabular}

Notes. ${ }^{(a)}$ See Table 6 of Paper I. ${ }^{(b)}$ When available. If not available we used $T_{\mathrm{e}}([\mathrm{O} \mathrm{III}])$.

we used the adopted density obtained from the CEL ratios for each object (see Table 1) and a set of $I(\mathrm{He} \mathrm{I}) / I(\mathrm{HI})$ line ratios. For all objects, we employed at least 12 observational constraints (He I lines $+n_{\mathrm{e}}$ ); each of which depends upon the four unknown quantities, and each dependence is unique. Finally, we obtained the best value for the four unknowns and the temperature fluctuations parameter, $t^{2}$ (see Sect. 5.1), by minimizing $\chi^{2}$. The obtained $\chi^{2}$ parameters are showed in Table 1; these parameters indicate a reasonable goodness of the fits, taking into account the degrees of freedom in each case.

Several He II emission lines were measured in the spectra of Hb 4, M 1-25, M 1-32, NGC 5189, NGC 6369, PC 14, and $\mathrm{Pe} 1-1$. We used the brightest lines to compute the $\mathrm{He}^{++} / \mathrm{H}^{+}$ratio using the recombination coefficients computed by Storey \& Hummer (1995). There is a very good agreement between the results obtained from the different lines, therefore we finally adopted the $\mathrm{He}^{++} / \mathrm{H}^{+}$average, weighted by the uncertainties of each individual line. Final results are presented in Table 1. For the remaining objects, which have a lower ionization degree, we detected no He II lines.

\subsection{Heavy-element ionic abundances from CELs}

We derived the ionic abundance ratios, $X^{+i} / \mathrm{H}^{+}$, for several heavy-element ions from CELs. Computations were made using PYNEB $^{3}$ (Luridiana et al. 2012), a python based package that generalizes the tasks of the IRAF package NEBULAR, except for $\mathrm{Fe}, \mathrm{Ni}$, and $\mathrm{Ca}$ ions, for which we used our own scripts. As pointed out in Paper I, we assumed for each object a three-zone ionization scheme, where temperatures are represented by $T_{\mathrm{e}}([\mathrm{N} \mathrm{II}]), T_{\mathrm{e}}([\mathrm{O} \mathrm{III}])$ and $T_{\mathrm{e}}([\mathrm{Ar} \mathrm{IV}])$, when available, for the low-, medium- and high-ionization zones, respectively. In Table 2 we summarize the physical conditions used for each ion. In general, we used $T_{\mathrm{e}}([\mathrm{N}$ II $])-n_{\mathrm{e}}$ (low to medium) for ions with ionization potential, IP $<17 \mathrm{eV}$; and $T_{\mathrm{e}}$ ([O III])- $n_{\mathrm{e}}$ (low to medium) for ions with $17 \mathrm{eV}<\mathrm{IP}<39 \mathrm{eV}$. For high-ionization species, those with IP $>39 \mathrm{eV}$ (see Table 2), we have computed the abundances taking into account two sets of physical conditions: set A, which adopts $T_{\mathrm{e}}([\mathrm{O} \mathrm{III}])$ and $n_{\mathrm{e}}$ (low to medium) and set $\mathrm{B}$, which uses $T_{\mathrm{e}}\left([\mathrm{Ar}\right.$ IV $]$ ) and $n_{\mathrm{e}}$ (high). Note that this only affects the $\mathrm{Cl}, \mathrm{Ne}$, and $\mathrm{Ar}$ ionic abundances. We discuss the results in Sect. 3. The lines selected to compute ionic abundances are showed in Table 3 . The ionic abundances obtained are presented in Table 4. Errors in the ionic abundances were computed from the uncertainties in the line fluxes through Monte Carlo simulations.

The atomic data used for our computations are the same as in Paper I and are presented in Table 6 of García-Rojas et al. (2009) except for the transition probabilities of [Ne III], [Ne IV], and [ $\mathrm{Ne} \mathrm{V}]$, which were updated to the values given by Galavis et al. (1997), Becker et al. (1989), and Galavis et al. (1997),

\footnotetext{
3 http://www.iac.es/proyecto/PyNeb
}

Table 3. Lines used for abundance determinations.

\begin{tabular}{|c|c|}
\hline Ion & Line \\
\hline $\mathrm{N}^{+}$ & {$[\mathrm{N}$ II] $\lambda \lambda 6548,6584$} \\
\hline $\mathrm{O}^{+}$ & [O II] $\lambda \lambda 3726+29,7320+30$ \\
\hline $\mathrm{O}^{++}$ & {$[\mathrm{O}$ III $] \lambda \lambda 4959,5007$} \\
\hline $\mathrm{Ne}^{++}$ & {$[\mathrm{Ne}$ III] $\lambda \lambda 3868,3967$} \\
\hline $\mathrm{Ne}^{+3}$ & {$[\mathrm{Ne}$ IV] $\lambda \lambda 4714+15,4724+25$} \\
\hline $\mathrm{Ne}^{+4}$ & {$[\mathrm{Ne} \mathrm{v}] \lambda 3425$} \\
\hline$S^{+}$ & [S II] $\lambda \lambda 6717+31,4068+76$ \\
\hline $\mathrm{S}^{++}$ & [S III] $\lambda 9069$ \\
\hline $\mathrm{Cl}^{+}$ & [Cl II] $\lambda \lambda 8578,9123$ \\
\hline $\mathrm{Cl}^{++}$ & {$[\mathrm{Cl} \mathrm{III}] \lambda 5517+37$} \\
\hline $\mathrm{Cl}^{+3}$ & [Cl IV] $\lambda 8045$ \\
\hline $\mathrm{Ar}^{++}$ & [Ar III] $\lambda \lambda 7136,7751$ \\
\hline $\mathrm{Ar}^{+3}$ & {$[$ Ar IV] $\lambda \lambda 4711+40$} \\
\hline $\mathrm{Ar}^{+4}$ & [Ar v] $\lambda 7005$ \\
\hline $\mathrm{Fe}^{+}$ & [Fe II] $\lambda 8616$ (7155) \\
\hline $\mathrm{Fe}^{++}$ & {$[\mathrm{Fe}$ III] $\lambda \lambda 4658,4701,4733,4754,4769,4881$} \\
\hline $\mathrm{Fe}^{+3}$ & {$[\mathrm{Fe}$ IV $] \lambda 6740$} \\
\hline $\mathrm{Ni}^{++}$ & [Ni III] $\lambda \lambda 6000,6401,6533$ \\
\hline $\mathrm{Ca}^{+}$ & {$[\mathrm{Ca}$ II $] \lambda \lambda 7291,7324$} \\
\hline
\end{tabular}

respectively, and the transition probabilities of [S II] and [S III] that were updated to the values provided by Podobedova et al. (2009). For the $\mathrm{Fe}, \mathrm{Ni}$, and $\mathrm{Ca}$ ions, the atomic data are described below.

Several [Fe II] lines have been detected in our spectra. Most of them are affected by continuum fluorescence effects (Rodríguez 1999; Verner et al. 2000). Fortunately, in the objects Cn 1-5, He 2-86, M 1-32, and Pe 1-1 we detected the [Fe II] $\lambda 8616$ line, which is almost insensitive to the effects of UV pumping. In addition, for M 1-30 and PC 14, we estimated the $\mathrm{Fe}^{+}$abundance using the [Fe II] $\lambda 7155$ line, which is least sensitive to fluorescence effects apart from the [Fe II] 18616 line (Verner et al. 2000). For these two objects, we assumed $I(\lambda 7155) / I(\lambda 8616) \sim 1$ (Rodríguez 1996). To compute the final $\mathrm{Fe}^{+}$abundances we used the atomic data presented in Bautista \& Pradhan (1998) and we resolved a 159-level atom.

At least one, and in most of the cases, several [Fe III] lines unaffected by fluorescence, were detected in our PNe. Only the spectrum of NGC 6369 did not present any [Fe III] line. To calculate the $\mathrm{Fe}^{++} / \mathrm{H}^{+}$ratio, we implemented a 34-level model atom that uses collision strengths from Zhang (1996) and the transition probabilities of Quinet (1996) as well as the new transitions found by Johansson et al. (2000). The average value of the $\mathrm{Fe}^{++}$ abundance was obtained from several individual emission lines for the different objects, except for Hb 4, M 3-15, and NGC 5189, where only one (Hb 4) or two lines (M3-15 and NGC 5189) were measured.

One [Fe IV] line was detected in He 2-86 at $\lambda 6739.79$. The $\mathrm{Fe}^{+3} / \mathrm{H}^{+}$ratio was derived using a 33-level model atom where all collision strengths are those calculated by Zhang \& Pradhan (1997) and the transition probabilities are those recommended by Froese Fischer \& Rubin (1998), and those from Garstang (1958) for the transitions not considered by Froese Fischer \& Rubin.

Several [Ni II] lines have been measured in several objects, but they are strongly affected by continuum fluorescence (Lucy 1995). To compute $\mathrm{Ni}^{+}$abundances it is necessary to use a 76-level model that includes continuum fluorescence excitation. Therefore, a proper description of the ionizing continuum in the zone covered by the slit is necessary for each $\mathrm{PNe}$, that is, we would need to compute the distance between the central star and 
Table 4. Ionic abundances from CELs.

\begin{tabular}{|c|c|c|c|c|c|c|c|}
\hline \multirow[b]{2}{*}{ Ion } & \multicolumn{7}{|c|}{$12+\log \left(\mathrm{X}^{+i} / \mathrm{H}^{+}\right)$} \\
\hline & $\mathrm{Cn} 1-5$ & $\mathrm{Hb} 4$ & $\mathrm{He} 2-86$ & M1-25 & M1-30 & M1-32 & M1-61 \\
\hline $\mathrm{N}^{+}$ & $8.03 \pm 0.04$ & $7.41 \pm 0.08$ & $7.48 \pm 0.07$ & $8.01 \pm 0.06$ & $8.34 \pm 0.05$ & $8.25 \pm 0.08$ & $6.95 \pm 0.07$ \\
\hline $\mathrm{O}^{+}$ & $8.09 \pm 0.07$ & $7.56 \pm 0.12$ & $7.64 \pm 0.13$ & $8.48 \pm 0.12$ & $8.65 \pm 0.11$ & $8.54 \pm 0.15$ & $7.35 \pm 0.14$ \\
\hline $\mathrm{O}^{++}$ & $8.69 \pm 0.04$ & $8.61 \pm 0.04$ & $8.75 \pm 0.04$ & $8.63 \pm 0.04$ & $8.53 \pm 0.05$ & $8.27 \pm 0.04$ & $8.65 \pm 0.04$ \\
\hline $\mathrm{Ne}^{++a}$ & $8.27 \pm 0.04$ & $8.07 \pm 0.06 / 7.63 \pm 0.12$ & $8.23 \pm 0.05 / 8.43 \pm 0.12$ & $7.47 \pm 0.10$ & $7.67 \pm 0.08$ & $7.23 \pm 0.06$ & $8.05 \pm 0.05 / 8.32 \pm 0.19$ \\
\hline $\mathrm{Ne}^{+3 a}$ & - & - & - & - & - & - & - \\
\hline $\mathrm{Ne}^{+4 a}$ & - & - & - & - & - & - & - \\
\hline $\mathrm{S}^{+}$ & $6.37 \pm 0.08$ & $5.65 \pm 0.43$ & $5.90 \pm 0.14$ & $6.31 \pm 0.18$ & $6.33 \pm 0.12$ & $6.56 \pm 0.18$ & $5.55 \pm 0.10$ \\
\hline $\mathrm{S}^{++}$ & $7.00 \pm 0.05$ & $6.73 \pm 0.08$ & $6.95 \pm 0.08$ & $7.12 \pm 0.06$ & $7.17 \pm 0.07$ & $7.02 \pm 0.10$ & $6.80 \pm 0.08$ \\
\hline $\mathrm{Cl}^{+}$ & $4.89 \pm 0.05$ & $4.33 \pm 0.11$ & $4.40 \pm 0.08$ & $4.74 \pm 0.08$ & $4.93 \pm 0.08$ & $5.06 \pm 0.11$ & $4.09 \pm 0.08$ \\
\hline $\mathrm{Cl}^{++}$ & $5.40 \pm 0.04$ & $5.04 \pm 0.06$ & $5.32 \pm 0.05$ & $5.42 \pm 0.05$ & $5.54 \pm 0.05$ & $5.25 \pm 0.06$ & $5.05 \pm 0.06$ \\
\hline $\mathrm{Cl}^{+3}$ & $3.94 \pm 0.06$ & $4.79 \pm 0.08 / 4.55 \pm 0,10$ & $4.50 \pm 0.06 / 4.60 \pm 0.08$ & $2.88 \pm 0.10$ & - & - & $4.28 \pm 0.06 / 4.42 \pm 0.11$ \\
\hline $\mathrm{Ar}^{++}$ & $6.61 \pm 0.04$ & $6.33 \pm 0.07$ & $6.52 \pm 0.13$ & $6.64 \pm 0.05$ & $6.74 \pm 0.06$ & $6.53 \pm 0.06$ & $6.39 \pm 0.05$ \\
\hline $\mathrm{Ar}^{+3 a}$ & $5.11 \pm 0.11$ & $6.04 \pm 0.05 / 5.66 \pm 0.11$ & $5.57 \pm 0.07 / 5.78 \pm 0.11$ & - & $4.42 \pm 0.25$ & $4.35 \pm 0.23$ & $5.39 \pm 0.08 / 5.65 \pm 0.17$ \\
\hline $\mathrm{Ar}^{+4 a}$ & - & $4.32 \pm 0.07 / 4.02 \pm 0.10$ & - & - & - & - & - \\
\hline $\mathrm{Fe}^{+}$ & $5.36 \pm 0.10$ & - & $4.72 \pm 0.13$ & $5.10 \pm 0.12$ & $4.65 \pm 0.10$ & $6.05 \pm 0.16$ & $4.13 \pm 0.14$ \\
\hline $\mathrm{Fe}^{++}$ & $5.95 \pm 0.06$ & $4.78 \pm 0.25$ & $5.52 \pm 0.07$ & $5.87 \pm 0.07$ & $5.49 \pm 0.07$ & $6.56 \pm 0.08$ & $4.80 \pm 0.11 / 4.85 \pm 0.07$ \\
\hline $\mathrm{Fe}^{+3}$ & - & - & $5.73 \pm 0.13$ & - & - & - & - \\
\hline $\mathrm{Ni}^{++}$ & $4.68 \pm 0.11$ & - & $4.38 \pm 0.32$ & _- & $4.28 \pm 0.11$ & $5.54 \pm 0.11$ & - \\
\hline $\mathrm{Ca}^{+}$ & - & - & $2.19 \pm 0.23$ & $2.66 \pm 0.12$ & - & $3.85 \pm 0.12$ & - \\
\hline Ion & M3-15 & NGC5189 & NGC6369 & PC14 & Pe1-1 & PB8 & NGC2867 \\
\hline $\mathrm{N}^{+}$ & $6.75 \pm 0.08$ & $8.12 \pm 0.05$ & $6.51 \pm 0.05$ & $6.81 \pm 0.05$ & $7.39 \pm 0.07$ & $6.80 \pm 0.08$ & $6.92 \pm 0.04$ \\
\hline $\mathrm{O}^{+}$ & $7.23 \pm 0.11$ & $8.28 \pm 0.13$ & $6.88 \pm 0.08$ & $7.43 \pm 0.09$ & $8.00 \pm 0.13$ & $7.36 \pm 0.13$ & $7.40 \pm 0.07$ \\
\hline $\mathrm{O}^{++}$ & $8.80 \pm 0.05$ & $8.42 \pm 0.04$ & $8.52 \pm 0.04$ & $8.74 \pm 0.04$ & $8.56 \pm 0.04$ & $8.75 \pm 0.05$ & $8.44 \pm 0.04$ \\
\hline $\mathrm{Ne}^{++a}$ & $8.01 \pm 0.14$ & $7.96 \pm 0.06 / 7.46 \pm 0.10$ & $7.89 \pm 0.04 / 7.53 \pm 0.16$ & $8.17 \pm 0.05 / 7.67 \pm 0.21$ & $7.92 \pm 0.05$ & $8.11 \pm 0.06$ & $7.80 \pm 0.05$ \\
\hline $\mathrm{Ne}^{+3 a}$ & - & $7.91 \pm 0.30 / 6.80 \pm 0.36$ & - & - & - & - & $7.57 \pm 0.28$ \\
\hline $\mathrm{Ne}^{+4 a}$ & - & $6.46 \pm 0.06 / 5.88 \pm 0.11$ & - & - & - & - & $5.48 \pm 0.06$ \\
\hline $\mathrm{S}^{+}$ & $5.35 \pm 0.25$ & $6.57 \pm 0.07$ & $5.22 \pm 0.06$ & $5.56 \pm 0.10$ & $6.02 \pm 0.12$ & $5.08 \pm 0.08$ & $5.52 \pm 0.05$ \\
\hline $\mathrm{S}^{++}$ & $6.82 \pm 0.09$ & $6.88 \pm 0.06$ & $6.50 \pm 0.05$ & $6.77 \pm 0.05$ & $6.61 \pm 0.06$ & $6.98 \pm 0.07$ & $6.37 \pm 0.05$ \\
\hline $\mathrm{Cl}^{+}$ & $3.91 \pm 0.11$ & $4.99 \pm 0.06$ & $3.83 \pm 0.08$ & $4.08 \pm 0.09$ & $4.45 \pm 0.07$ & - & - \\
\hline $\mathrm{Cl}^{++}$ & $5.20 \pm 0.06$ & $5.10 \pm 0.04$ & $4.90 \pm 0.04$ & $5.10 \pm 0.04$ & $5.01 \pm 0.06$ & $5.29 \pm 0.08$ & $4.80 \pm 0.05$ \\
\hline $\mathrm{Cl}^{+3}$ & $4.45 \pm 0.08$ & $4.47 \pm 0.05 / 4.17 \pm 0.07$ & $4.40 \pm 0.04 / 4.20 \pm 0.10$ & $4.63 \pm 0.04 / 4.34 \pm 0.12$ & $3.77 \pm 0.05$ & - & $4.40 \pm 0.04$ \\
\hline $\mathrm{Ar}^{++}$ & $6.40 \pm 0.07$ & $6.43 \pm 0.05$ & $6.17 \pm 0.04$ & $6.27 \pm 0.04$ & $6.31 \pm 0.05$ & $6.58 \pm 0.05$ & $5.96 \pm 0.04$ \\
\hline $\mathrm{Ar}^{+3 a}$ & $5.68 \pm 0.09$ & $5.82 \pm 0.04 / 5.38 \pm 0.09$ & $5.44 \pm 0.05 / 5.14 \pm 0.14$ & $5.70 \pm 0.05 / 5.27 \pm 0.18$ & $4.55 \pm 0.18$ & $4.98 \pm 0.15$ & $5.63 \pm 0.06$ \\
\hline $\mathrm{Ar}^{+4 a}$ & - & $4.82 \pm 0.05 / 4.46 \pm 0.08$ & - & - & - & - & $4.40 \pm 0.07$ \\
\hline $\mathrm{Fe}^{+}$ & - & - & - & $3.87 \pm 0.23$ & $4.71 \pm 0.16$ & - & - \\
\hline $\mathrm{Fe}^{++}$ & $5.04 \pm 0.13$ & $4.40 \pm 0.17$ & - & $4.62 \pm 0.11$ & $4.98 \pm 0.13$ & - & - \\
\hline $\mathrm{Fe}^{+3}$ & - & - & - & - & - & - & - \\
\hline $\mathrm{Ni}^{++}$ & - & - & - & - & - & - & - \\
\hline $\mathrm{Ca}^{+}$ & - & - & - & - & $2.54 \pm 0.17$ & - & - \\
\hline
\end{tabular}

Notes. ${ }^{(a)}$ For these ions we computed abundances considering low to medium ionization and high-ionization physical conditions when different (see text).

the area covered by our slit, in addition to the stellar radius and effective temperature. All these requirements are very difficult to be carried out for most of our objects, given the uncertainties in the distances to the PNe and that many of them are almost completely covered by our slit. Because of the relatively low importance of this ion in the total $\mathrm{Ni}$ abundance, even for lowionization objects such as $\mathrm{H}$ II regions (see Mesa-Delgado et al. 2009 b), we finally did not compute $\mathrm{Ni}^{+}$abundances for our PNe, because in general they are more highly ionized objects than average $\mathrm{H}$ II regions.

We measured several [Ni III] lines in our PNe. These lines are not expected to be affected by fluorescence given the structure of the ion (Bautista 2001), in the same way as the Fe III lines. The $\mathrm{Ni}^{++} / \mathrm{H}^{+}$ratio was derived using a 126-level model atom and the atomic data of Bautista (2001).

Two [Ca II] lines at $\lambda \lambda 7291$ and 7324 were detected in the spectrum of He 2-86 and only one at $\lambda 7291$ in M 1-25, M 1-32 and $\mathrm{Pe} 1-1$. The [Ca II] line at $\lambda 7291$ in $\mathrm{He} 2-86$ was not reported in Paper I; we obtained an intensity of $I([\mathrm{Ca} I \mathrm{II}]) / I(\mathrm{H} \beta)=$ $0.0095 \pm 0.0013(I(\mathrm{H} \beta)=100)$ for this object. To derive the $\mathrm{Ca}^{+}$ abundance, we solved a five-level model atom using the single atomic data set available for this ion (Meléndez et al. 2007). Note that this is the first determination of the $\mathrm{Ca}^{+}$abundance in a PNe ever, and, because we did not detect additional ionization stages of this ion, this places a lower limit to the gas-phase $\mathrm{Ca} / \mathrm{H}$ ratio in these objects.

\subsection{Heavy-element ionic abundances from ORLs}

Multiple permitted lines of heavy-element ions, such as C I, C II, C III, N I, N II, N III, O I, O II, O III, Ne I, Ne II, Si I, Si II, S II, $\mathrm{S}$ III, $\mathrm{Cl}$ I, and $\mathrm{Mg}$ II were detected in our spectra. Many of these lines are affected by fluorescence effects, as has been pointed out in García-Rojas et al. (2009, and references therein) or are blended with strong telluric emissions, making their intensities unreliable.

Several of these detected permitted lines are heavy-element ORLs, mainly of O I, O II, C II, C III, and N II, but also of Ne II and $\mathrm{Mg}$ II, therefore we were able to measure ionic abundances of the corresponding ions. Most of these lines are detected for the first time in these PNe. To derive abundances we used the atomic data compiled in Table 6 of García-Rojas et al. (2009) 
for O II, C II, C III, N II, and Ne II ORLs. For O I and Mg II the atomic data are described below.

We followed a similar strategy as was used in previous papers (see García-Rojas et al. 2009; García-Rojas \& Esteban 2007, and references therein), selecting the most adequate multiplets of each ion to compute the ionic abundace from ORLs.

\subsubsection{Permitted lines of oxygen}

We detected and measured one or several lines of multiplet 1 of $\mathrm{O}$ I in several objects. Unfortunately, these lines are in a spectral range with strong telluric $\mathrm{OH}$ emission lines that frequently blur the $\mathrm{O}$ I lines. In Fig. 1 we show the spectral section where O I RLs lie in the seven objects they have been detected in. We were easily able to deblend the three lines in M 1-30; in Pe 1-1 we were able to deblend O I $\lambda 7771.94$ from a telluric line and measured a blend of the other two components of the multiplet; in M 1-32 and NGC 5189 we were able to properly measure two lines; in Cn 1-5, He 2-86 we were able only to properly measure one line; and in M 1-25 we were able to measure only a blend of the whole multiplet with the telluric line. For Pe 1-1 we applied the theoretical ratio O I $\lambda 7774.17 / \lambda 7775.39$ to deblend both lines and derive individual abundances. For M 1-25, it was impossible to deblend the $\mathrm{O}$ I lines from the telluric line at $\sim 7774 \AA$, therefore we decided to integrate the entire flux of the three $\mathrm{O}$ I lines plus the telluric line between two given limits and then used this value to compute an upper limit to the abundance. In Table 5 we present the abundances obtained from each single line and from the sum of all available lines of the multiplet. The adopted $\mathrm{O}^{+} / \mathrm{H}^{+}$ratio is the average of the abundances obtained using the recombination coefficients from two atomic data sources (Pequignot et al. 1991; Escalante \& Victor 1992). The results obtained are shown in Table 5.

Many O II permitted lines were detected in the spectra of our PNe. We only considered those belonging to multiplets 1 , $2,10,20$, and from $3 \mathrm{~d}-4 \mathrm{f}$ transitions, which are mainly excited by recombination (Esteban et al. 2004, and references therein); however, some authors did not discard contributions by resonance fluorescense to these lines on relatively low-ionized objects, such as the Orion nebula and the PN IC 418 (Escalante \& Morisset 2005; Escalante et al. 2012, see below). In Fig. 2 we show the region of the multiplet 1 O II ORLs for each PN of the sample. The intensity of the brightest lines of this multiplet is about $10^{-3} \times I(\mathrm{H} \beta)$, which indicates the quality of the spectra. Tsamis et al. (2003) and Ruiz et al. (2003) pointed out that the upper levels of the transitions of multiplet 1 of $\mathrm{O}$ II are not in local thermodynamic equilibrium (LTE) for densities $n_{e}<10^{4} \mathrm{~cm}^{-3}$, which is the case for several of our WRPNe, and the abundances derived from each individual line may differ by factors as large as 4 . We applied the non-LTE corrections estimated by Peimbert et al. (2005) to our data, and the abundances obtained from the individual lines agree well between them; we also derived the abundance using the sum of all lines of the multiplet following the recipe given by Esteban et al. (1998). This abundance, which is not affected by non-LTE effects, agrees with that derived from individual lines. In Table 6 we present the values obtained for the individual lines, as well as that derived from the sum of all the lines of a given multiplet. We only considered lines with errors lower than $40 \%$; for $3 \mathrm{~d}-4 \mathrm{f}$ transitions, when available, we averaged lines with errors $<40 \%$, otherwise, we averaged all available lines and quoted the final value with an uncertainty higher than $40 \%$. O II $\lambda \lambda 4097.26,4294.92$ and
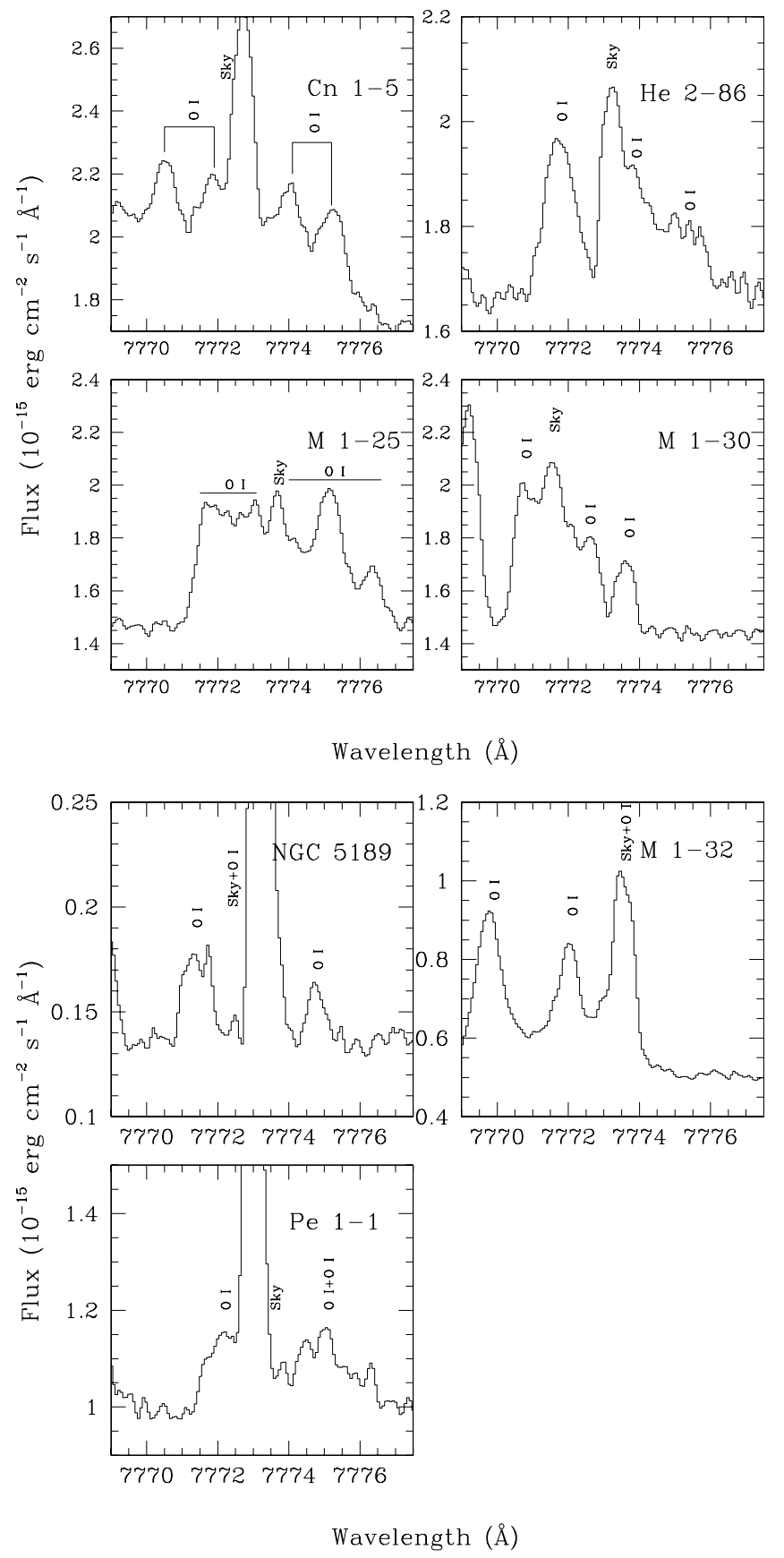

Fig. 1. Section of the echelle spectra showing the recombination emission lines of multiplet 1 of $\mathrm{O}$ I. The relative position of the telluric emission line changes from one PN to the next due to the different heliocentric velocities of the PNe.

4613.68 were not taken into account because they are severely blended with other lines.

In Fig. 3 we show the $\mathrm{O}^{++}$abundances derived for multiplets $1,2,10$, and 20 (sum value) and for 3d-4f transitions (averaged value) for each object of our sample. In general, all values agree within the uncertainties for the best signal-to-noise spectra, where more $\mathrm{O}$ II lines were detected and measured, that is, He 2-86 and M 1-30. For PC 14, abundances from multiplets 1 and 20 agree, but are somewhat different from those of multiplets 2 (lower) and multiplet 10 and $3 \mathrm{~d}-4 \mathrm{f}$ transitions (higher). However, the behaviour of multiplet 2 can be explained because the brightest line of the multiplet, that is, O II $\lambda 4349.43$, gives abundances systematically lower than the rest of the lines of the 
Table 5. Ionic abundance ratios from the O I recombination lines.

\begin{tabular}{|c|c|c|c|c|c|c|c|c|c|c|c|c|c|}
\hline \multirow[t]{2}{*}{ Mult. } & \multirow[t]{2}{*}{$\lambda_{0}$} & \multicolumn{12}{|c|}{$\mathrm{O}^{+} / \mathrm{H}^{+}\left(\times 10^{-5}\right)$} \\
\hline & & Cn 1-5 & $\mathrm{Hb} 4$ & He 2-86 & M 1-25 & M 1-30 & M 1-32 & M 1-61 & M3-15 & NGC 5189 & NGC 6369 & PC 14 & Pe $1-1$ \\
\hline \multirow[t]{5}{*}{1} & 7771.94 & $34 / 27$ & - & $18 / 14$ & $41 / 32$ & $96 / 74$ & $80 / 61$ & - & - & $26 / 20$ & - & - & $22: / 17$ : \\
\hline & 7774.17 & - & - & - & $41 / 32$ & $110 / 85$ & $75 / 58$ & - & - & - & - & - & 17:/13: \\
\hline & 7775.39 & - & - & - & $41 / 32$ & $95 / 73$ & - & - & - & 26:/20: & - & - & $22: / 17$ : \\
\hline & Sum & $34 / 27$ & - & $18 / 14$ & $41 / 32$ & $101 / 77$ & $78 / 60$ & - & - & $26 / 20$ & - & - & 20:/16: \\
\hline & Adopted & $31 \pm 11$ & - & $16 \pm 4$ & $<37:$ & $89 \pm 20$ & $67 \pm 22$ & - & - & $23 \pm 8$ & - & - & 18: \\
\hline
\end{tabular}

Notes. Only lines with intensity uncertainties lower than $40 \%$ were considered (see text). Recombination coefficients from Escalante \& Victor (1992) and Pequignot et al. (1991).
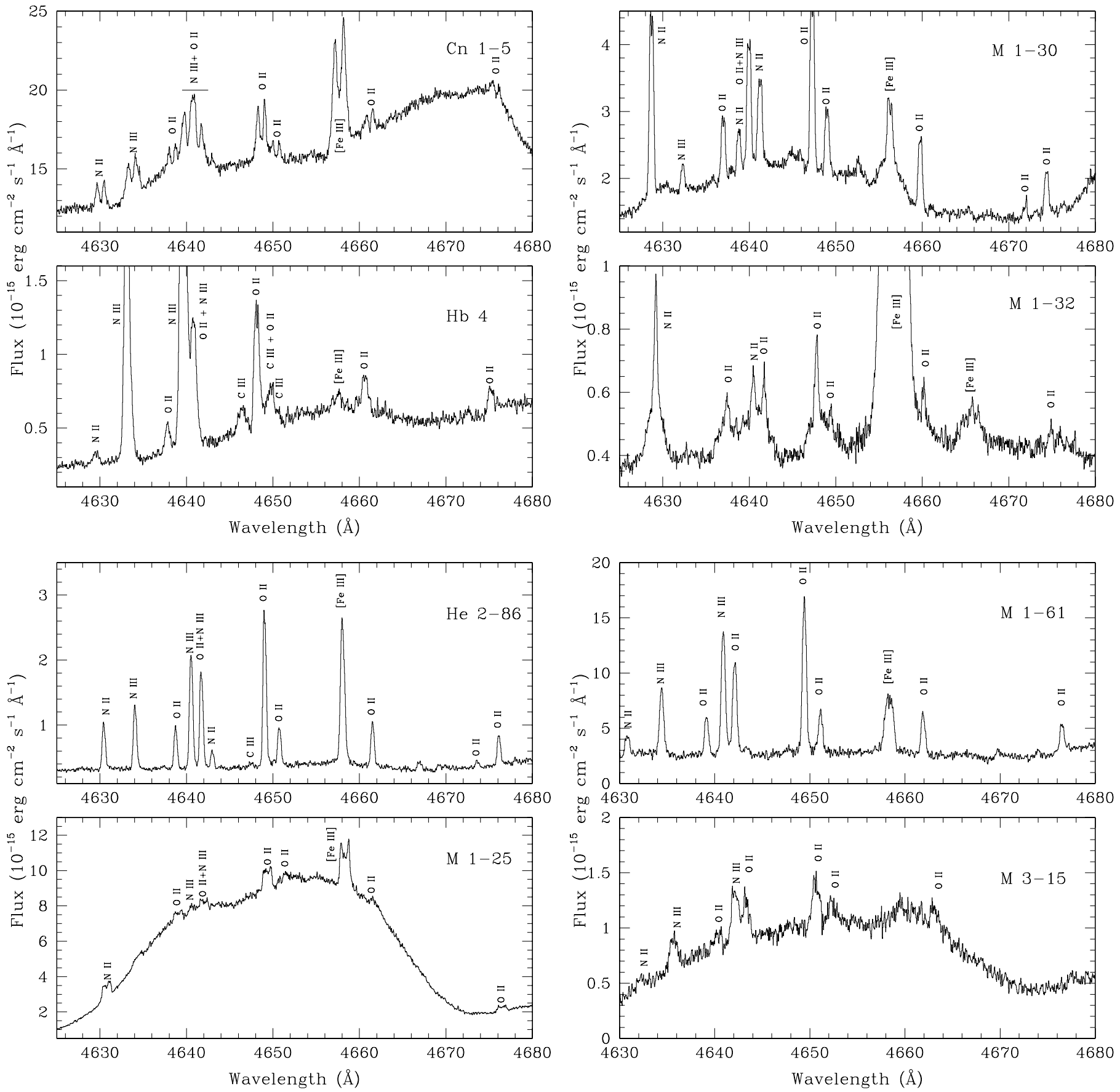

Fig. 2. Section of the echelle spectra showing the recombination emission lines of multiplet 1 of $\mathrm{O}$ II and multiplet 1 of C III. N II and N III emission lines in these plot are not excited by pure recombination (see text). 
J. García-Rojas et al.: PNe with [WC] central stars. II.
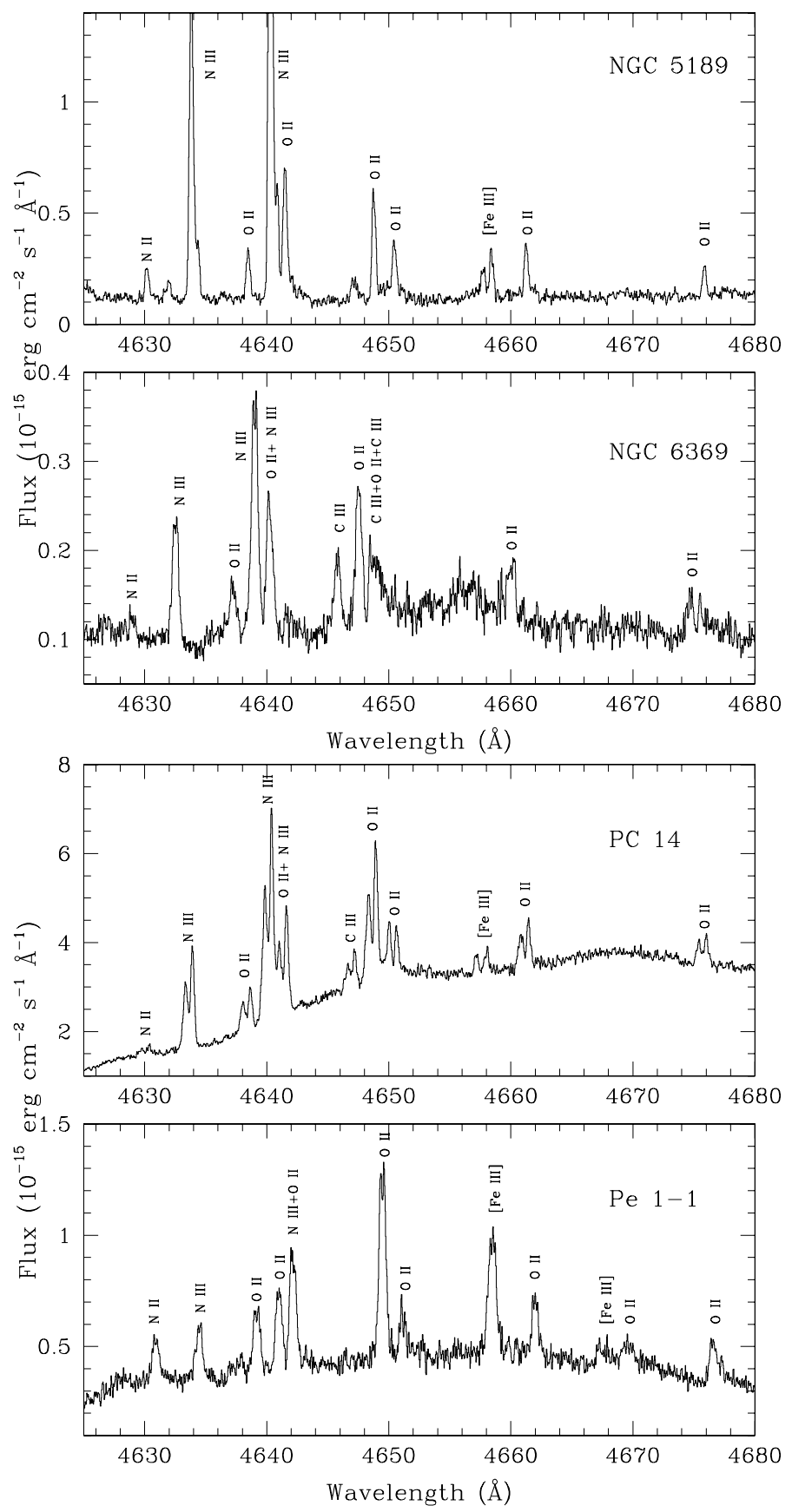

Fig. 2. continued.

multiplet, when measured properly. This can be caused by departures of the LTE in the populations of the levels where the O II multiplet 2 lines originate, in a similar way to what occurs for multiplet 1 (see Tsamis et al. 2003; Ruiz et al. 2003; Peimbert \& Peimbert 2005); unfortunately, the quality of the observations in the literature for the $\mathrm{O}$ II multiplet 2 lines is lower than that of the O II multiplet 1 ones, and an empirical recipe to correct for nonLTE effects could not be achieved. For the rest of objects there is an overall good agreement between the different multiplets within the uncertainties, except in the particular case of Pe 1-1, where multiplets 1 and 10 give abundances that are more than $1 \sigma$ different from the average abundance. For this PNe we adopted the value given by multiplet 1 as representative of the $\mathrm{O}^{++}$abundance from ORLs, giving the abundance with the lowest relative
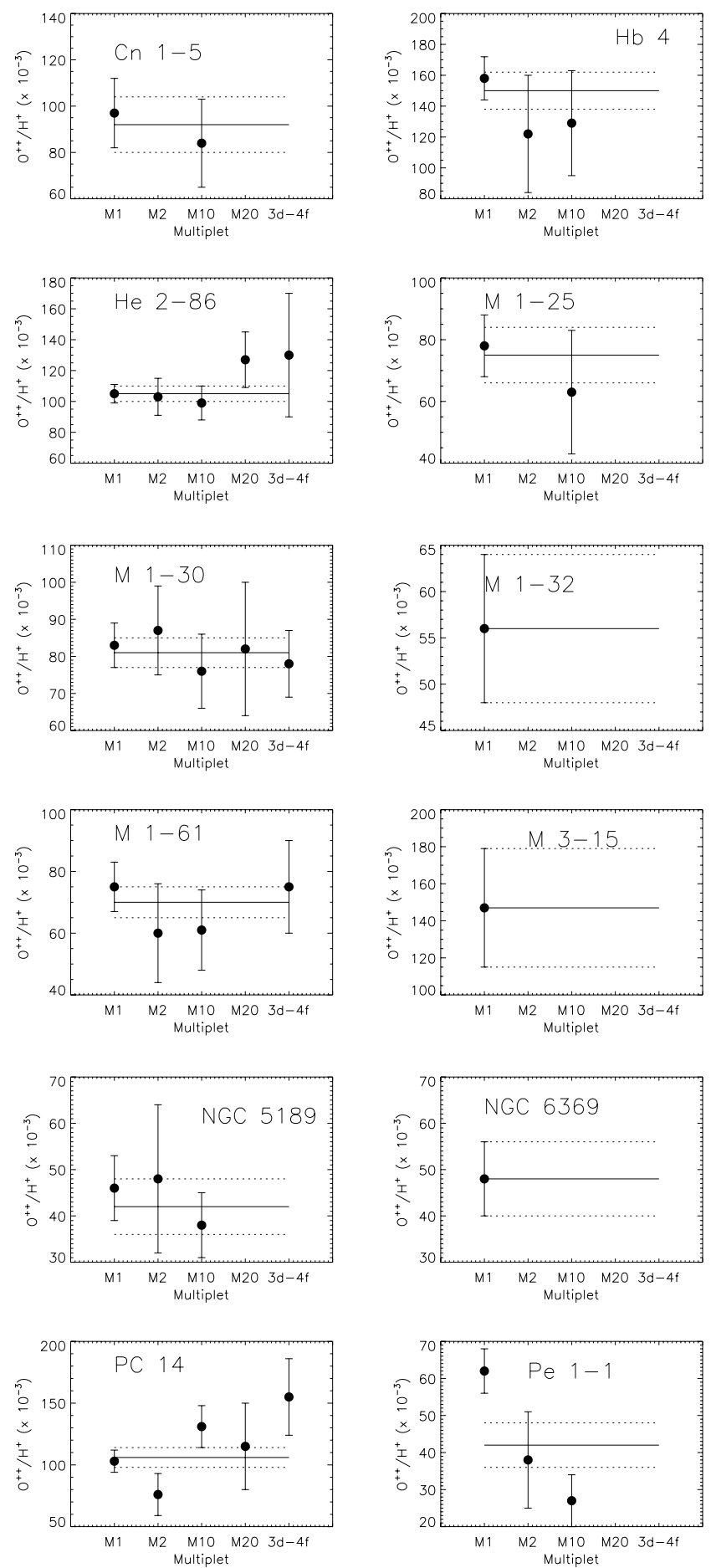

Fig. 3. $\mathrm{O}^{++} / \mathrm{H}^{+}$ratio obtained from different ORL multiplets for PNe of the sample. In general, the values obtained from the different multiplets are consistent. An exception is Pe 1-1 (see text). Solid lines represent the averaged value and dashed lines the $1 \sigma$ limits to the abundance.

uncertainties. Additional arguments favouring this value are presented in Sect. 5.1.

Very recently, Escalante et al. (2012) have shown that resonance fluorescence is probably responsible for about 10-20\% of the intensity of lines of multiplets 1,2 , and 20 of $\mathrm{O}$ II in the low-ionization PN IC 418, but this only affects a few (fewer than $5 \%$ ) lines of multiplet 10 and does not affect the lines of $3 d-4 f$ transitions at all. This result is striking because it calls into question the reliability of $\mathrm{O}$ II lines as abundance indicators, mainly 
in $\mathrm{H}$ II regions and in low-ionization PNe. In our sample, this effect is mitigated because of the high ionization degrees of most of the PNe.

We detected several lines of multiplets 2 and 5 of $\mathrm{O}$ III in the spectra of the most excited PNe of our sample. We did not consider these lines to compute abundances because they are probably excited by other mechanisms than recombination (see Grandi 1976; García-Rojas et al. 2009).

\subsubsection{Permitted lines of carbon}

Several permitted lines of C II were measured in the spectra of our PNe. All detected multiplets are 3d-4f transitions and are, in principle, excited by pure recombination (see Grandi 1976). In two objects, M 1-32 and NGC 5189, the intensity of multiplet 17.04 C II $\lambda 6461.95$ line was not reliable because it was affected by charge transfers in the $\mathrm{CCD}$. The $\mathrm{C}^{++} / \mathrm{H}^{+}$ratios obtained are shown in Table 7.

We detected lines of multiplets 1,16 , and 18 of C III in several objects of our sample. The excitation mechanism of these multiplets is pure recombination (see García-Rojas et al. 2009, and references therein), therefore they are suitable for ionic abundance determinations. Results are shown in Table 8.

\subsubsection{Permitted lines of neon}

We detected several permitted lines of Ne II in our objects, belonging to multiplets 1,39 , and 55 . Transitions from multiplet 1 and 55 are probably results of recombination because they correspond to quartets and their ground level has a doublet configuration (Esteban et al. 2004). However, the multiplet $1 \mathrm{Ne}$ II $\lambda 3694.22$ line gives abundances much higher than those derived from the multiplet 55 lines; because the multiplet 55 lines correspond to 3d-4f transitions, whose upper levels can hardly be populated by fluorescense, and the multiplet 1 lines correspond to $3 \mathrm{~s}-3 \mathrm{p}$ transitions, we rely more on the multiplet 55 values. Multiplet $39 \mathrm{Ne}$ II $\lambda 3829.77$ was detected only in M 1-30; this line corresponds to an intercombination transition $\left(3 \mathrm{p}^{2} \mathrm{P}^{0}-3 \mathrm{~d}^{4} \mathrm{D}\right)$ and the abundance derived from it is much higher than that derived from multiplet 55 , hence, it is probably excited by other mechanisms than recombination. To derive $\mathrm{Ne}^{++}$ abundances, we used effective recombination coefficients from recent calculations by Kisielius \& Storey (unpublished), assuming LS-coupling. We adopted the sum value derived from multiplet 55 as representative of the $\mathrm{Ne}^{++}$abundance. This is the first time that $\mathrm{Ne}^{++} / \mathrm{H}^{+}$has been derived from recombination lines for all these PNe.

\subsubsection{Permitted lines of nitrogen}

Many N II permitted lines, belonging to different multiplets, were measured in our spectra. Grandi (1976), Escalante \& Morisset (2005), and, very recently, Escalante et al. (2012) have discussed the formation mechanism of several permitted lines of N II in the Orion nebula and the PN IC 418, and concluded that recombination cannot account for the observed intensities of most of them, because resonance fluorescence by line and starlight excitation are the dominant mechanisms. Moreover, Liu et al. (2001) suggested that continuum fluorescence by starlight could be the excitation mechanism of several permitted lines of N II in some PNe. This can be especially important for multiplet 3, for which the resonance fluorescence contributes $\sim 85 \%$ and $\sim 75 \%$ in the Orion nebula and IC 418 , respectively (Escalante \& Morisset 2005; Escalante et al. 2012). On the other hand, the upper term of the $\mathrm{N}$ II $\lambda 4236.91, \lambda 4237.05$ and $\lambda 4241.78$ lines of multiplet 48 is $4 \mathrm{f}^{3} \mathrm{~F}$, and of the $\mathrm{N}$ II $\lambda 4041.31$ line of multiplet 39 is $4 \mathrm{f} G[9 / 2]$ and cannot be populated by permitted resonance transitions, therefore this line is probably excited mainly by recombination. For the multiplet 48 lines, we co-added the intensities of these lines, along with the characteristic wavelength of the whole multiplet, $4239.40 \AA$, to compute the abundance. The $\mathrm{N}^{++} / \mathrm{H}^{+}$ratios are shown in Table 10 .

The effect of fluorescense needs to be treated with caution because it depends on several factors: the fluorescense contribution is strongest in low-ionization PNe and, as the fluorescence contribution to a given line is emitted from the recombined ion, its level is related to the presence of this ion. In our case, the relative strength of the $\mathrm{N}^{+}$ions depends on the object, and we can only neglect the fluorescence in the emission of the $\mathrm{N}$ II lines for objects with a high ionization degree; additionally, the strength of the effect also depends on the form of the spectral energy distribution (SED); in Fig. 1 of Escalante et al. (2012) we can see that a detailed high-resolution model of the ionizing continuum and of the velocity field is needed to compute the exact contribution of fluorescence excitation. The stellar temperature and the form of the SED are reflected in the ionization degree of the PN. Given the complexity of fluorescence, the biases of our sample and that it deserves a detailed treatment for each object, it is beyond the scope of this paper to analyse this in detail. However, taking into account the quality of our data, some discussion is of interest. In Fig. 4 we present $\mathrm{N}^{++}$ abundance ratios obtained from different multiplets and methods: i) from multiplet 3 ; ii) from multiplet 39 ; iii) from multiplet 48; and iv) assuming that the ratios $\mathrm{N}^{++} / \mathrm{N}^{+}$and $\mathrm{O}^{++} / \mathrm{O}^{+}$ are similar and are also similar computed from CELs or ORLs (i. e. $\left.\mathrm{N}^{++} / \mathrm{H}^{+}\right)_{\text {ORLs }}=\left(\mathrm{N}^{+} / \mathrm{O}^{+}\right)_{\mathrm{CELs}} \times\left(\mathrm{O}^{++} / \mathrm{H}^{+}\right)_{\text {ORLs }}$. The first result is evident: there is a large scatter among the different values at a given ionization degree. Multiplet 3, M3, yields the highest values and multiplet 39, M39, the lowest ones. The tendencies of the different ratios also give us some information: apparently $\mathrm{N}^{++}(\mathrm{M} 3) / \mathrm{N}^{++}(\mathrm{M} 39)$ increases with the ionization degree, while $\mathrm{N}^{++}(\mathrm{M} 39) / \mathrm{N}^{++}$(M48) decreases; this behaviour could be explained taking into account the relative strength of fluorescence in IC 418, which increases from M39 (nothing), M48 $(\sim 20 \%)$ to M3 $(\sim 75 \%)$ and, as we have pointed out above, it is stronger at low-ionization degrees. However, the ratio $\mathrm{N}^{++}$ (M3)/ $\mathrm{N}^{++}$(M39) should show a similar increasing tendency, but the dispersion of the values does not let us confirm this beyond doubt. Finally, the values obtained from the assumption that $\left(\mathrm{N}^{++} / \mathrm{H}^{+}\right)_{\mathrm{ORLs}}=\left(\mathrm{N}^{+} / \mathrm{O}^{+}\right)_{\mathrm{CELs}} \times\left(\mathrm{O}^{++} / \mathrm{H}^{+}\right)_{\mathrm{ORLs}}$ compared with abundances derived from multiplet 3 do not match any of the values reported here from permitted lines. Therefore, the $\mathrm{N}^{++}$abundances obtained from permitted lines should be treated carefully, especially when correcting for the recombination contribution to the auroral [N II] $\lambda 5755$ line (Paper I).

We did not consider the lines of multiplets 1 and 2 of $\mathrm{N}$ III detected in the spectra of several objects because they appear to be excited by the Bowen mechanism and are not reliable for abundance determinations (Grandi 1976).

\subsubsection{Permitted lines of magnesium}

We detected the recombination Mg II 3d-4f $\lambda 4481.21$ in four objects of our sample. However, in three of them the line is near the detection limit, therefore abundances derived from this line need to be treated with some caution. Following Liu et al. (2004), we took advantage of the similarity of the C II and $\mathrm{Mg}$ II atomic 
J. García-Rojas et al.: PNe with [WC] central stars. II.

Table 6. Ionic abundance ratios from the O II recombination lines.

\begin{tabular}{|c|c|c|c|c|c|c|c|c|c|c|c|c|c|}
\hline \multirow[t]{2}{*}{ Mult. } & \multirow[t]{2}{*}{$\lambda_{0}$} & \multicolumn{12}{|c|}{$\mathrm{O}^{++} / \mathrm{H}^{+}\left(\times 10^{-5}\right)$} \\
\hline & & Cn $1-5$ & $\mathrm{Hb} 4$ & He 2-86 & M 1-25 & M 1-30 & M 1-32 & M 1-61 & M3-15 & NGC 5189 & NGC 6369 & $\mathrm{PC} 14$ & Pe 1-1 \\
\hline \multirow[t]{9}{*}{$1^{a}$} & 4638.85 & 43: & 79 & 102 & 83 & 78 & 125: & 67 & 139: & 32 & 44 & 88 & 78 \\
\hline & 4641.81 & - & 185 & 102 & 71 & 83 & 88: & 74 & 144 & 52 & 57 & 97 & 56 \\
\hline & 4649.14 & 96 & 189 & 109 & 70 & 88 & 57 & 77 & 136 & 96 & 56 & 124 & 61 \\
\hline & 4650.84 & 34: & 184 & 110 & 77 & 80 & 53 & 77 & 183 & 30 & 33 & 87 & 38: \\
\hline & 4661.64 & 77 & 110 & 100 & 113 & 77 & 38: & 70 & 121: & 34 & 42 & 88 & 60 \\
\hline & 4673.73 & - & - & 105 & - & 84: & - & - & - & - & - & - & - \\
\hline & 4676.24 & 140 & 124 & 100 & 80 & 89 & 105: & 78 & - & 59 & 41: & 130 & 70 \\
\hline & 4696.14 & - & - & 140 & - & - & - & - & - & - & - & - & - \\
\hline & Sum & $97 \pm 15$ & $158 \pm 14$ & $105 \pm 6$ & $78 \pm 10$ & $83 \pm 6$ & $56 \pm 8$ & $75 \pm 8$ & $147 \pm 32$ & $46 \pm 7$ & $48 \pm 8$ & $103 \pm 9$ & $62 \pm 6$ \\
\hline \multirow[t]{8}{*}{2} & 4317.14 & 71: & 105: & 122 & 100: & 113 & $373:^{f}$ & 84: & - & 55: & - & 88: & 43 \\
\hline & 4319.63 & 50: & - & 115 & 60: & 67 & $372:^{f}$ & 63: & - & - & - & 72: & 32 \\
\hline & 4325.76 & - & - & 160 & 320: & 211: & - & 202: & - & - & - & 339: & 338: \\
\hline & 4336.83 & - & - & 161 & 107: & 118 & - & 103: & - & - & - & 137: & 73: \\
\hline & $4345.56^{d}$ & 117: & 58: & 136 & 128: & 161: & 95: & 104: & - & 34: & 28: & 91: & 79: \\
\hline & 4349.43 & 52: & 89 & 68 & 48: & 58 & 57: & 49 & - & 32 & 16: & 68 & 41: \\
\hline & 4366.89 & 93: & 206 & 100 & 92: & 146 & - & 88 & - & 89 & - & 98 & 95: \\
\hline & Sum & 63: & $122 \pm 38$ & $103 \pm 12$ & 84: & $87 \pm 12$ & 67: & $60 \pm 16$ & - & $48 \pm 16$ & 19: & $76 \pm 17$ & $38 \pm 13$ \\
\hline \multirow[t]{8}{*}{$10^{c}$} & 4069.62 & 83 & 160 & 110 & 63 & 80 & - & 70 & 142: & $162^{b}$ & - & 165 & 29 \\
\hline & 4069.89 & $*$ & $*$ & $*$ & $*$ & $*$ & * & $*$ & $*$ & $*$ & * & $*$ & $*$ \\
\hline & 4072.15 & 85 & 115 & 94 & 63 & 71 & - & 57 & - & 59 & - & 116 & 26 \\
\hline & 4075.86 & 85 & 115 & 94 & 63 & 71 & - & 57 & 110: & 22 & 91: & 116 & 26 \\
\hline & 4078.84 & 134: & 134 & 134 & - & 120 & - & 161: & - & - & - & 151: & - \\
\hline & 4085.11 & - & - & 83 & - & 83 & - & 117: & - & 88: & - & 156: & - \\
\hline & 4092.93 & - & - & 102 & - & - & - & - & - & - & - & 96: & - \\
\hline & Sum & $84 \pm 19$ & $129 \pm 34$ & $99 \pm 11$ & $63 \pm 20$ & $76 \pm 10$ & - & $61 \pm 13$ & 124: & $38 \pm 7$ & 91: & $131 \pm 17$ & $27 \pm 7$ \\
\hline \multirow[t]{5}{*}{$20^{c}$} & $4097.22^{b}$ & - & - & - & - & - & - & - & - & - & - & - & - \\
\hline & 4104.79 & - & - & 115 & 82: & 100 & - & 103: & - & - & - & 54: & - \\
\hline & $4110.79^{e}$ & 165: & - & 206: & 193: & 318: & - & 128: & - & - & - & - & - \\
\hline & 4119.22 & - & 148: & 132 & 69: & 65 & - & 66: & - & $52:$ & - & 115 & - \\
\hline & Sum & 165: & 148: & $127 \pm 18$ & 91: & $82 \pm 18$ & - & 87: & - & 52: & - & $115 \pm 35$ & - \\
\hline \multirow[t]{22}{*}{$3 d-4 f^{c}$} & 4083.90 & - & - & 143 & - & - & - & - & - & - & - & 146: & - \\
\hline & 4087.15 & - & - & 147 & - & 116 & - & - & - & 113: & - & 173: & - \\
\hline & 4089.29 & 74: & - & 126 & $56:$ & 65 & - & 72 & - & 40: & - & 126: & 63: \\
\hline & 4095.64 & - & - & 212 & - & - & - & - & - & - & - & 167: & - \\
\hline & $4097.26^{b}$ & - & - & - & - & - & - & - & - & - & - & - & 218: \\
\hline & 4275.55 & 120: & 95: & 171 & - & 82 & 184: & 77 & - & - & - & 77: & - \\
\hline & 4276.75 & 118: & 94: & 95 & - & - & 182: & 79 & - & - & - & 93: & - \\
\hline & 4277.90 & - & - & - & - & - & - & - & - & - & - & 163: & - \\
\hline & 4282.96 & - & - & - & - & 59: & - & - & - & - & - & 109: & - \\
\hline & 4285.69 & - & - & 115 & - & - & - & 75: & - & - & - & 112: & - \\
\hline & 4288.82 & - & - & $608^{e}$ & - & - & - & - & - & - & - & - & - \\
\hline & 4291.25 & - & - & 95: & - & 69: & - & - & - & - & - & 97: & - \\
\hline & 4292.21 & - & - & 156: & - & 205: & - & - & - & - & - & - & - \\
\hline & $4294.78^{b}$ & - & - & 112 & - & - & - & - & - & - & - & - & - \\
\hline & 4303.61 & 108: & 79: & 122 & $75:$ & 87 & - & $62:$ & - & 82: & - & 156 & - \\
\hline & 4303.82 & 106: & 152: & 124 & 70: & 84 & - & 56: & - & 86: & - & 150 & - \\
\hline & 4491.23 & 230: & - & 114 & 131: & 181: & - & - & - & 231: & - & 259: & 283: \\
\hline & 4602.13 & - & - & 224 & - & - & - & - & - & - & - & 130: & - \\
\hline & 4609.44 & - & 172: & 109 & 58: & 87 & - & 56: & - & - & - & 159 & - \\
\hline & $4613.68^{b}$ & - & - & - & - & - & - & - & - & - & - & - & - \\
\hline & Average & 126: & 118: & $130 \pm 40$ & 78: & $78 \pm 9$ & 183: & $75 \pm 15$ & - & 110: & - & $155 \pm 31$ & \\
\hline & Adopted & $92 \pm 12$ & $150 \pm 12$ & $105 \pm 5$ & $75 \pm 9$ & $81 \pm 4$ & $56 \pm 8$ & $70 \pm 7$ & $147 \pm 32$ & $42 \pm 6$ & $48 \pm 8$ & $106 \pm 8$ & $62 \pm 6$ \\
\hline
\end{tabular}

Notes. Only lines with intensity uncertainties lower than $40 \%$ have been considered (see text). ${ }^{(a)}$ Corrected for non-LTE effects (see text). ${ }^{(b)}$ Blended with another line or affected by internal reflections or charge transfer in the CCD. ${ }^{(c)}$ Recombination coefficients for intermediate coupling (Liu et al. 1995). ${ }^{(d)}$ Blended with the O II 4345.55 line. ${ }^{(e)}$ Blended with an unknown line. ${ }^{(f)}$ Lines are at the very edge of one order and the overlapping between orders was unsuitable.

structure and assumed that the Mg II 3d-4f $\lambda 4481.21$ line has an effective recombination coefficient equal to that of the $\mathrm{C}$ II $3 \mathrm{~d}-4 \mathrm{f}$ $\lambda 4267$ line. Therefore, from the $\mathrm{Mg}$ II $\lambda 4481.21 / \mathrm{H} \beta$ intensity ratio and the $\mathrm{C}$ II line effective recombination coefficient we can derive $\mathrm{Mg}^{++} / \mathrm{H}^{+}$, which is shown for each PN in Table 11 .

\section{Total abundances}

To correct for the unseen ionization stages and then derive the total gaseous abundances of chemical elements in our PNe, we should have adopted a set of ionization correction factors (ICF). For oxygen and nitrogen, we adopted the classical ICF scheme reported by Kingsburgh \& Barlow (1994), but for the other elements, special cases need to be discussed. In Table 12 we show the total abundances obtained for our sample.

\subsection{Helium}

When He II lines were detected, the total helium is the sum of $\mathrm{He}^{+}$and $\mathrm{He}^{++}$, otherwise we used the $\mathrm{ICF}(\mathrm{He})$ reported by 
Table 7. Ionic abundance ratios from the $\mathrm{C}$ II recombination lines.

\begin{tabular}{lccccccccccccc}
\hline \hline Mult. & \multicolumn{1}{c}{$\lambda_{0}$} & \multicolumn{10}{c}{$\mathrm{C}^{++} / \mathrm{H}^{+}\left(\times 10^{-5}\right)$} \\
\hline & & Cn 1-5 & Hb 4 & He 2-86 & M 1-25 & M 1-30 & M 1-32 & M 1-61 & M 3-15 & NGC 5189 & NGC 6369 & PC 14 & Pe 1-1 \\
\hline 6 & 4267.15 & 130 & 72 & 68 & 53 & 86 & 192 & 43 & 50 & 29 & 77 & 83 & 107 \\
17.06 & 5342.38 & 174 & 49 & 73 & 62 & 92 & 276 & 50 & 82 & - & - & 66 & 104 \\
16.04 & 6151.43 & 130 & 56 & 63 & 53 & 78 & 157 & 47 & 85 & 50 & 101 & 72 & 112 \\
17.04 & 6461.95 & 92 & 74 & 59 & 55 & 90 & $84^{a}$ & 43 & 69 & $56^{a}$ & 87 & 77 & 98 \\
\hline & Adopted & $\mathbf{1 2 1} \pm \mathbf{7}$ & $\mathbf{6 8} \pm \mathbf{6}$ & $\mathbf{6 5} \pm \mathbf{3}$ & $\mathbf{5 6} \pm \mathbf{3}$ & $\mathbf{8 6} \pm \mathbf{3}$ & $\mathbf{1 9 4} \pm \mathbf{1 4}$ & $\mathbf{4 4} \pm \mathbf{3}$ & $\mathbf{6 9} \pm \mathbf{7}$ & $\mathbf{3 0} \pm \mathbf{3}$ & $\mathbf{8 1} \pm \mathbf{6}$ & $\mathbf{8 1} \pm \mathbf{5}$ & $\mathbf{1 0 4} \pm \mathbf{6}$ \\
\hline
\end{tabular}

Notes. Only lines with intensity uncertainties lower than $40 \%$ were considered (see text). ${ }^{(a)}$ Affected by charge transfer in the CCD.

Table 8. Ionic abundance ratios from the C III recombination lines.

\begin{tabular}{|c|c|c|c|c|c|c|c|c|c|c|c|c|c|}
\hline \multirow[t]{2}{*}{ Mult. } & \multirow[t]{2}{*}{$\lambda_{0}$} & \multicolumn{12}{|c|}{$\mathrm{C}^{+3} / \mathrm{H}^{+}\left(\times 10^{-5}\right)$} \\
\hline & & Cn 1-5 & $\mathrm{Hb} 4$ & He 2-86 & M 1-25 & M 1-30 & M 1-32 & M 1-61 & M 3-15 & NGC 5189 & NGC 6369 & $\mathrm{PC} 14$ & Pe 1-1 \\
\hline \multirow[t]{4}{*}{1} & 4647.42 & - & 34 & 4 & - & - & - & $3:$ & - & $6:$ & 17 & 19 & - \\
\hline & 4650.25 & - & 34 & 4 & - & - & - & - & - & - & 17 & - & - \\
\hline & 4651.47 & - & 31: & - & - & - & - & - & - & - & 17 & - & - \\
\hline & Sum & - & $34 \pm 7$ & $4 \pm 1$ & - & - & - & 3: & - & 6: & $17 \pm 4$ & $19 \pm 4$ & - \\
\hline \multirow[t]{4}{*}{16} & 4067.94 & - & - & - & - & - & - & - & - & - & 32 & - & - \\
\hline & $4068.91^{a}$ & - & - & - & - & - & - & - & - & 41: & 32 & - & - \\
\hline & 4070.26 & - & - & - & - & - & - & - & - & 24 & 32 & - & - \\
\hline & Sum & - & - & - & - & - & - & - & - & $24 \pm 4$ & $32 \pm 5$ & - & - \\
\hline \multirow[t]{2}{*}{18} & 4186.90 & - & 13: & - & - & - & - & - & - & 4: & - & $13 \pm 5$ & - \\
\hline & Adopted & - & $34 \pm 7$ & $4 \pm 1$ & - & - & - & 3: & - & $24 \pm 4$ & $23 \pm 3$ & $17 \pm 3$ & - \\
\hline
\end{tabular}

Notes. Only lines with intensity uncertainties lower than $40 \%$ were considered (see text). ${ }^{(a)}$ Deblended from [S II] $\lambda 4068.60$ by assuming the theoretical ratio C III $\lambda 4067.94 / \lambda 4068.91$.

Table 9. Ionic abundance ratios from the Ne II recombination lines.

\begin{tabular}{|c|c|c|c|c|c|c|c|c|c|c|c|c|c|}
\hline \multirow[t]{2}{*}{ Mult. } & \multirow[t]{2}{*}{$\lambda_{0}$} & \multicolumn{12}{|c|}{$\mathrm{Ne}^{++} / \mathrm{H}^{+}\left(\times 10^{-5}\right)$} \\
\hline & & Cn $1-5$ & $\mathrm{Hb} 4$ & He 2-86 & M 1-25 & M 1-30 & M 1-32 & M 1-61 & M3-15 & NGC 5189 & NGC 6369 & PC 14 & Pe 1-1 \\
\hline 1 & 3694.22 & 50 & - & - & 53 & - & - & 14: & - & 19 & - & 54 & - \\
\hline 39 & 3829.77 & - & - & - & - & 46 & - & - & - & - & - & - & - \\
\hline \multirow[t]{4}{*}{55} & 4391.94 & 17: & 33: & 16 & 25: & 10 & - & $6:$ & 54: & 12: & - & 16: & 7: \\
\hline & 4409.30 & $22:$ & 17: & 27 & 34: & 11: & - & 13: & $39:$ & 9: & - & 19: & - \\
\hline & Sum & 19: & 27: & $20 \pm 9$ & $28:$ & 10 & - & 9: & 49: & 11: & - & 17: & 7: \\
\hline & Adopted $^{a}$ & 19: & 27: & $20 \pm 9$ & 28: & 10: & - & 9: & 49: & 11: & - & 17: & 7: \\
\hline
\end{tabular}

Notes. ${ }^{(a)}$ Lines from multiplet 1 and 39 have not been considered (see text).

Table 10. Ionic abundance ratios from the $\mathrm{N}$ II recombination lines.

\begin{tabular}{|c|c|c|c|c|c|c|c|c|c|c|c|c|c|}
\hline \multirow[t]{2}{*}{ Mult. } & \multirow[t]{2}{*}{$\lambda_{0}$} & \multicolumn{12}{|c|}{$\mathrm{N}^{++} / \mathrm{H}^{+}\left(\times 10^{-5}\right)$} \\
\hline & & Cn 1-5 & $\mathrm{Hb} 4$ & He 2-86 & M 1-25 & M 1-30 & M 1-32 & M 1-61 & M3-15 & NGC 5189 & NGC 6369 & $\mathrm{PC} 14$ & Pe 1-1 \\
\hline \multirow[t]{6}{*}{$3^{a}$} & 5666.64 & 61 & 30 & 60 & 65 & 107 & 73 & 21 & 31 & 21 & - & 15 & 20 \\
\hline & 5676.02 & 50 & 28 & 50 & 51 & 98 & 63 & 18 & - & 15 & - & - & - \\
\hline & 5679.56 & 52 & 32 & 60 & 54 & 89 & 55 & 20 & 26 & 15 & 8.9 & 14 & 17 \\
\hline & 5686.21 & 65 & 34 & 50 & 58 & 102 & 96 & - & - & - & - & - & - \\
\hline & 5710.76 & 52 & 31 & 58 & 66 & 102 & 83 & 17 & - & - & - & - & - \\
\hline & Sum & $55 \pm 5$ & $31 \pm 4$ & $58 \pm 4$ & $58 \pm 4$ & $97 \pm 6$ & $66 \pm 9$ & $20 \pm 2$ & $29 \pm 4$ & $17 \pm 3$ & $8.9 \pm 3.2$ & $15 \pm 3$ & $18 \pm 2$ \\
\hline $39^{b}$ & 4041.31 & 19: & 11: & $21 \pm 4$ & 14: & $20 \pm 4$ & - & $6.5:$ & - & 7.1: & - & 8.3: & - \\
\hline \multirow[t]{2}{*}{$48^{c}$} & $4239.40^{c}$ & 41/30: & 20/14: & $40 / 29 \pm 6$ & $32 / 23:$ & $49 / 35 \pm 9$ & - & - & - & 22/16: & - & 10/7.4: & - \\
\hline & Adopted & 27: & 14: & $28 \pm 4$ & 21: & $31 \pm 4$ & $\leq 66$ & 6.5: & $\leq \mathbf{2 9}$ & 14: & $\leq 8.9$ & 8.5: & $\leq 18$ \\
\hline
\end{tabular}

Notes. ${ }^{(a)}$ Recombination coefficients reported by Kisielius \& Storey (2002). ${ }^{(b)}$ Recombination coefficients reported by Pequignot et al. (1991).

${ }^{(c)}$ We considered the sum of all lines of the multiplet. Recombination coefficients reported by Pequignot et al. (1991)/Escalante \& Victor (1992). 
Table 11. Ionic abundance ratios from the Mg II recombination lines.

\begin{tabular}{|c|c|c|c|c|c|c|c|c|c|c|c|c|c|}
\hline \multirow[t]{2}{*}{ Mult. } & \multirow[t]{2}{*}{$\lambda_{0}$} & \multicolumn{12}{|c|}{$\mathrm{Mg}^{++} / \mathrm{H}^{+}\left(\times 10^{-5}\right)$} \\
\hline & & Cn $1-5$ & $\mathrm{Hb} 4$ & He 2-86 & M 1-25 & M 1-30 & M 1-32 & M 1-61 & M3-15 & NGC 5189 & NGC 6369 & $\mathrm{PC} 14$ & Pe 1-1 \\
\hline 4 & 4481.21 & - & 5.02: & 4.45 & $3.14:$ & $2.66:$ & - & - & - & - & - & - & - \\
\hline & Adopted & - & 5.02: & $4.45 \pm 0.85$ & 3.14: & 2.66: & - & - & - & - & - & - & - \\
\hline
\end{tabular}

Notes. Recombination coefficient equal to that of the C II 3d-4f $\lambda 4267$ line (see text).

Table 12. Total abundances.

\begin{tabular}{|c|c|c|c|c|c|c|c|}
\hline \multirow[b]{2}{*}{ Element } & \multicolumn{7}{|c|}{$12+\log (\mathrm{X} / \mathrm{H})$} \\
\hline & Cn1-5 & $\mathrm{Hb} 4$ & $\mathrm{He} 2-86$ & M1-25 & M1-30 & M1-32 & M1-61 \\
\hline $\mathrm{He}$ & $11.21 \pm 0.01$ & $11.06 \pm 0.02$ & $11.09 \pm 0.01$ & $11.09 \pm 0.01$ & $11.12 \pm 0.01$ & $11.10 \pm 0.01$ & $11.05 \pm 0.01$ \\
\hline $\mathrm{C}^{a}$ & $9.18 \pm 0.05$ & $9.07 \pm 0.06$ & $8.87 \pm 0.06$ & $8.96 \pm 0.07$ & $9.30 \pm 0.07$ & $9.75 \pm 0.09$ & $8.68 \pm 0.06$ \\
\hline $\mathrm{N}$ & $8.72 \pm 0.05$ & $8.55 \pm 0.09$ & $8.62 \pm 0.09$ & $8.40 \pm 0.06$ & $8.59 \pm 0.05$ & $8.44 \pm 0.06$ & $8.28 \pm 0.11$ \\
\hline $\mathrm{O}$ & $8.79 \pm 0.03$ & $8.70 \pm 0.04$ & $8.79 \pm 0.04$ & $8.87 \pm 0.05$ & $8.90 \pm 0.06$ & $8.74 \pm 0.10$ & $8.67 \pm 0.04$ \\
\hline $\mathrm{O}^{b}$ & $9.07 \pm 0.04$ & $9.27 \pm 0.04$ & $9.08 \pm 0.02$ & $9.05 \pm 0.05$ & $9.23 \pm 0.05$ & $9.11 \pm 0.08$ & $8.89 \pm 0.04$ \\
\hline $\mathrm{Ne}^{c}$ & $8.37 \pm 0.04$ & $8.17 \pm 0.06 / 7.73 \pm 0.12$ & $8.27 \pm 0.05 / 8.46 \pm 0.12$ & $7.71 \pm 0.11$ & $8.04 \pm 0.08$ & $7.69 \pm 0.12$ & $8.08 \pm 0.07 / 8.34 \pm 0.19$ \\
\hline S & $7.20 \pm 0.04$ & $7.01 \pm 0.09$ & $7.23 \pm 0.08$ & $7.22 \pm 0.06$ & $7.25 \pm 0.07$ & $7.17 \pm 0.09$ & $7.11 \pm 0.09$ \\
\hline $\mathrm{Cl}$ & $5.53 \pm 0.03$ & $5.37 \pm 0.04 / 5.31 \pm 0.04$ & $5.43 \pm 0.04$ & $5.50 \pm 0.04$ & $5.63 \pm 0.04$ & $5.47 \pm 0.06$ & $5.17 \pm 0.05$ \\
\hline $\mathrm{Ar}^{c}$ & $6.73 \pm 0.04$ & $6.55 \pm 0.05 / 6.45 \pm 0.06$ & $6.61 \pm 0.12 / 6.63 \pm 0.11$ & $6.92 \pm 0.05$ & $7.11 \pm 0.08$ & $6.99 \pm 0.11$ & $6.45 \pm 0.05 / 6.49 \pm 0.05$ \\
\hline $\mathrm{Mg}^{d}$ & - & 7.70: & $7.65 \pm 0.08$ & $7.50:$ & $7.42:$ & - & - \\
\hline $\mathrm{Fe}^{e}$ & $6.05 \pm 0.05$ & - & $5.96 \pm 0.08$ & $5.94 \pm 0.06$ & $5.55 \pm 0.06$ & $6.68 \pm 0.07$ & $4.88 \pm 0.10$ \\
\hline $\mathrm{Fe}^{f}$ & $6.34 \pm 0.10$ & $5.35 \pm 0.30$ & $6.06 \pm 0.14$ & $6.21 \pm 0.14$ & $5.55 \pm 0.06$ & $6.68 \pm 0.07$ & $5.41 \pm 0.17$ \\
\hline $\mathrm{Ni}^{g}$ & $5.37 \pm 0.22$ & - & $5.56 \pm 0.38$ & - & $4.52 \pm 0.18$ & $5.72 \pm 0.19$ & - \\
\hline Element & M3-15 & NGC5189 & NGC6369 & $\mathrm{PC} 14$ & Pe1-1 & PB8 & NGC2867 \\
\hline $\mathrm{He}$ & $11.03 \pm 0.02$ & $11.09 \pm 0.01$ & $11.02 \pm 0.01$ & $11.03 \pm 0.01$ & $11.02 \pm 0.01$ & $11.09 \pm 0.01$ & $11.06 \pm 0.01$ \\
\hline $\mathrm{C}^{a}$ & $8.85 \pm 0.13$ & $9.02 \pm 0.10$ & $9.00 \pm 0.05$ & $9.02 \pm 0.04$ & $9.13 \pm 0.06$ & $8.85 \pm 0.03$ & $9.25 \pm 0.03$ \\
\hline $\mathrm{N}$ & $8.33 \pm 0.10$ & $8.60 \pm 0.07$ & $8.17 \pm 0.06$ & $8.15 \pm 0.07$ & $8.06 \pm 0.08$ & $8.20 \pm 0.08$ & $8.09 \pm 0.06$ \\
\hline $\mathrm{O}$ & $8.81 \pm 0.05$ & $8.77 \pm 0.06$ & $8.53 \pm 0.04$ & $8.77 \pm 0.04$ & $8.67 \pm 0.04$ & $8.77 \pm 0.05$ & $8.58 \pm 0.04$ \\
\hline $\mathrm{O}^{b}$ & $9.18 \pm 0.08$ & $8.92 \pm 0.07$ & $8.69 \pm 0.06$ & $9.06 \pm 0.04$ & $8.88 \pm 0.05$ & $9.11 \pm 0.02$ & $8.78 \pm 0.03$ \\
\hline $\mathrm{Ne}^{c}$ & $8.03 \pm 0.13$ & $8.27 \pm 0.13 / 7.57 \pm 0.13$ & $7.90 \pm 0.04 / 7.54 \pm 0.16$ & $8.20 \pm 0.04 / 7.70 \pm 0.21$ & $8.03 \pm 0.06$ & $8.13 \pm 0.06$ & $8.02 \pm 0.10$ \\
\hline S & $7.21 \pm 0.10$ & $7.11 \pm 0.05$ & $6.92 \pm 0.05$ & $7.09 \pm 0.06$ & $6.81 \pm 0.06$ & $7.30 \pm 0.09$ & $6.68 \pm 0.05$ \\
\hline $\mathrm{Cl}$ & $5.30 \pm 0.05$ & $5.57 \pm 0.03 / 5.54 \pm 0.03$ & $5.05 \pm 0.04 / 5.02 \pm 0.04$ & $5.27 \pm 0.03 / 5.22 \pm 0.04$ & $5.14 \pm 0.05$ & $5.29 \pm 0.08$ & $5.10 \pm 0.04$ \\
\hline $\mathrm{Ar}^{c}$ & $6.49 \pm 0.06$ & $6.71 \pm 0.06 / 6.65 \pm 0.06$ & $6.26 \pm 0.04 / 6.22 \pm 0.04$ & $6.39 \pm 0.04 / 6.33 \pm 0.04$ & $6.43 \pm 0.05$ & $6.61 \pm 0.05$ & $6.17 \pm 0.04$ \\
\hline $\mathrm{Mg}^{d}$ & - & - & - & - & - & - & - \\
\hline $\mathrm{Fe}^{e}$ & - & - & - & $4.69 \pm 0.10$ & $5.17 \pm 0.11$ & - & - \\
\hline $\mathrm{Fe}^{f}$ & $5.75 \pm 0.18$ & $4.84 \pm 0.23$ & - & $5.24 \pm 0.14$ & $5.36 \pm 0.18$ & - & - \\
\hline $\mathrm{Ni}^{g}$ & - & - & - & - & - & - & - \\
\hline
\end{tabular}

Notes. ${ }^{(a)}$ From recombination lines (see text). ${ }^{(b)}$ From recombination lines (see text). ${ }^{(c)}$ Considering low to medium ionization or high-ionization physical conditions when different (see text). ${ }^{(d)}$ From recombination lines (see text). ${ }^{(e)} \mathrm{Fe} / \mathrm{H}=\mathrm{Fe}^{+} / \mathrm{H}^{+}+\mathrm{Fe}^{++} / \mathrm{H}^{+}$, except for $\mathrm{He} 2-86$, where $\mathrm{Fe} / \mathrm{H}=\mathrm{Fe}^{+} / \mathrm{H}^{+}+\mathrm{Fe}^{++} / \mathrm{H}^{+}+\mathrm{Fe}^{+3} / \mathrm{H}^{+}$. ${ }^{(f)}$ ICF from an observational fit reported by Rodríguez \& Rubin $(2005)$. Valid for $-0.1<\log \left(\mathrm{O}^{+} / \mathrm{O}^{++}\right)<$ -1.35. ${ }^{(g)}$ Empirical ICF, following Mesa-Delgado et al. (2009b).

Peimbert et al. (1992). Their ICF is somewhat uncertain, but does not significantly affect the total He abundance, given the low amount of neutral He expected in our objects.

\subsection{Carbon}

For carbon, when C II and C III lines were detected and He II lines as well (Hb 4, NGC 5189, NGC 6369 and PC 14), we applied the ICF given by Leisy \& Dennefeld (1996). When C II and C III lines were detected, but no He II lines (He 2-86 and M 1-61) or when C II lines only are detected, we applied the recipe given by Kingsburgh \& Barlow (1994).

\subsection{Neon}

When [Ne III], [Ne IV] and [Ne V] lines were observed, we assumed that the total $\mathrm{Ne}$ is the sum of all the observed ionic species. When [Ne III] lines only were observed, we applied the ICF scheme reported by Kingsburgh \& Barlow (1994).

\subsection{Sulfur}

For sulfur, we used the recipe given by Stasińska (1978) from photoionization models, which is

$\frac{\mathrm{S}}{\mathrm{H}}=\left[1-\left(\frac{\mathrm{O}^{+}}{\mathrm{O}}\right)^{\alpha}\right]^{\left(\frac{-1}{\alpha}\right)} \times\left(\frac{\mathrm{S}^{+}+\mathrm{S}^{++}}{\mathrm{H}^{+}}\right)$,

where $\alpha=3$. Kwitter \& Henry (2001) developed a grid of detailed photoionization models including state-of-the-art atomic data as well as charge transfer and dielectronic recombination processes and found a more sophisticated ICF expression that, for the range of ionization degrees of our sample, gives total abundances similar to those derived using Stasińska (1978) expression. We finally adopted the expression by Stasińska (1978) because it is widely used and can be more useful to achieve meaningful comparisons with the literature. 


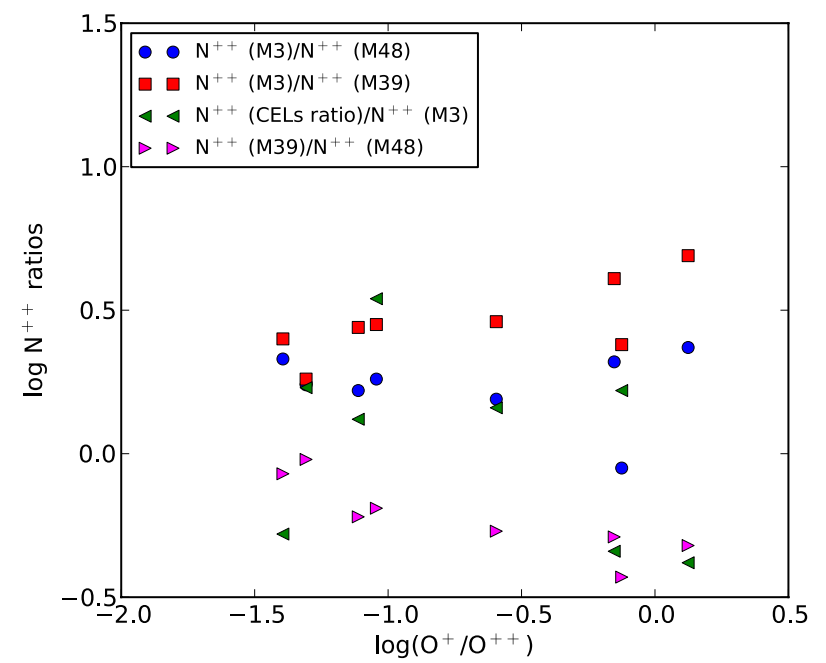

Fig. 4. $\mathrm{N}^{++}$ratios between different multiplets plotted against the ionization degree. The value $\mathrm{N}^{++}$(CELs ratio) corresponds to $\mathrm{N}^{++}$computed from the relation $\left(\mathrm{N}^{++} / \mathrm{H}^{+}\right)_{\text {ORLs }}=\left(\mathrm{N}^{+} / \mathrm{O}^{+}\right)_{\text {CELs }} \times\left(\mathrm{O}^{++} / \mathrm{H}^{+}\right)_{\text {ORLs }}$. Evidently, the dispersion of the values obtained from different multiplets of $\mathrm{N}^{++}$is very large, but some trends seem to be present and are discussed in the text.

\subsection{Chlorine}

The total abundance of $\mathrm{Cl}$ was considered as the sum of all ionic species: $\mathrm{Cl}^{+}, \mathrm{Cl}^{++}$and $\mathrm{Cl}^{+3}$ in objects with $\log \left(\mathrm{O}^{+} / \mathrm{O}^{++}\right)>-0.1$; in these objects chlorine is not expected to be more highly ionized. However, for the most ionized objects of our sample we computed the total abundance of $\mathrm{Cl}$ by using the ICF from Kwitter \& Henry (2001) to take into account the presence of $\mathrm{Cl}^{+4}$. Hence, the total chlorine was computed from

$$
\frac{\mathrm{Cl}}{\mathrm{H}}=\left(\frac{\mathrm{He}^{+}+\mathrm{He}^{++}}{\mathrm{He}^{+}}\right) \times\left(\frac{\mathrm{Cl}^{+}+\mathrm{Cl}^{++}+\mathrm{Cl}^{+3}}{\mathrm{H}^{+}}\right) .
$$

This scheme was also applied when no $\mathrm{Cl}^{+}$was detected in the PN. As a general result, this ICF gives total abundances that are similar to that derived from the simple sum of all ionic species, except for the cases of the high ionized PNe Hb 4 and NGC 5189 for which the abundances increase by 0.09 and $0.16 \mathrm{dex}$, respectively. For the lowest-ionized objects (M 1-30 and M 1-32), where only $\mathrm{Cl}^{+}$and $\mathrm{Cl}^{++}$are seen, we assumed that the total abundance of $\mathrm{Cl}$ is the sum of these two species. For PB 8, where the $\mathrm{Cl}^{++}$abundance only could be computed we assumed the ICF scheme proposed by Girard et al. (2007), which is

$$
\frac{\mathrm{Cl}}{\mathrm{H}}=\left(\frac{\mathrm{He}}{\mathrm{He}^{+}}\right)^{2} \times \frac{\mathrm{Cl}^{++}}{\mathrm{H}^{+}} \text {. }
$$

\subsection{Argon}

For Ar, we applied the recipe given by Kingsburgh \& Barlow (1994) when [Ar III] lines only were detected, or when [Ar III], [Ar IV] and [Ar V] were detected. These authors do not give any recipe when [Ar III] and [Ar IV] only are detected, which is the case for most of our objects. In such cases, we followed Wang \& Liu (2007), who used the expression of Kingsburgh \& Barlow (1994) when [Ar III], [Ar IV] and [Ar V] are detected, because they considered that the fraction of $[\mathrm{Ar} \mathrm{V}]$ is negligible which is, indeed, our case.

\subsection{Magnesium}

The total abundances of $\mathrm{Mg}$ were estimated from recombination lines. Following Liu et al. (2004), we considered that most of the $\mathrm{Mg}$ is in the form of $\mathrm{Mg}^{++}$for the excitation classes of the PNe where the faint $\mathrm{Mg}$ II ORL was detected. Therefore, we assumed $\mathrm{Mg} / \mathrm{H}=\mathrm{Mg}^{++} / \mathrm{H}^{+}$.

\subsection{Iron}

We measured lines of one, two, or three Fe ionization stages $\left(\mathrm{Fe}^{+}, \mathrm{Fe}^{++}\right.$and $\left.\mathrm{Fe}^{+3}\right)$ in our PNe. For He 2-86, we can derive the total $\mathrm{Fe}$ abundance from the sum of the three ionization stages. For the other objects - and also for He 2-86 for comparison we used an ICF to obtain the total $\mathrm{Fe} / \mathrm{H}$ ratio. Rodríguez \& Rubin (2005) suggested a correction scheme for iron based on two ICFs, one from photoionization models (their Eq. (2)) and one from an observational fit that takes into account all the uncertainties in the atomic data involved in the calculations and that allows us to constrain real iron abundances. Here, we only used the ICF from observational data given by

$\frac{\mathrm{Fe}}{\mathrm{H}}=1.1 \times\left(\frac{\mathrm{O}^{+}}{\mathrm{O}^{++}}\right)^{0.58} \times \frac{\mathrm{Fe}^{++}}{\mathrm{O}^{+}} \times \frac{\mathrm{O}}{\mathrm{H}}$,

which is valid in the range $-1.35<\log \left(\mathrm{O}^{+} / \mathrm{O}^{++}\right)<-0.1$. For $\log \left(\mathrm{O}^{+} / \mathrm{O}^{++}\right)>-0.1$ and, as $\mathrm{Fe}^{++}$and $\mathrm{O}^{+}$become the dominant ions, the ICF used is

$\frac{\mathrm{Fe}}{\mathrm{O}}=\frac{\mathrm{Fe}^{+}+\mathrm{Fe}^{++}}{\mathrm{O}^{+}}$

In Fig. 5 we present the $\mathrm{Fe} / \mathrm{H}$ ratio plotted against the ionization degree, which shows no particular tendency. From this plot, we can see that the iron abundances, $12+\log (\mathrm{Fe} / \mathrm{H})$, derived for our sample range from $4.84 \pm 0.23$ to $6.68 \pm 0.07$, which are well below the solar value $\left((\mathrm{Fe} / \mathrm{H})_{\odot}=7.46 \pm 0.08\right.$, Lodders 2010$)$ as a consequence of iron depletion into dust grains. We obtained that more than $85 \%$ of the iron atoms are deposited into dust grains, in agreement with previous findings in PNe (Delgado-Inglada et al. 2009). If we had used the ICF from photoionization models from Rodríguez \& Rubin (2005), the derived iron abundances would be up to 0.69 dex higher, but our conclusions about iron depletions would be similar, with more than $80 \%$ of the iron atoms in the nebulae condensed onto dust grains.

\subsection{Nickel}

There is no ICF available in the literature to correct for the presence of $\mathrm{Ni}^{+3}$ to calculate the total $\mathrm{Ni}$ abundance for the four PNe where we detected Ni emission lines. However, we followed the procedure developed by Mesa-Delgado et al. (2009b) to compute the total Ni abundance in the Orion nebula, who applied a firstorder ICF scheme based on the similarity between the ionization potentials of $\mathrm{Ni}^{+3}(35.17 \mathrm{eV})$ and $\mathrm{O}^{++}(35.12 \mathrm{eV})$ :

$\frac{\mathrm{Ni}^{+3}}{\mathrm{Ni}}=\frac{\mathrm{O}^{++}}{\mathrm{O}}$.

Therefore, they used the following expression to compute the total $\mathrm{Ni}$ abundance

$\frac{\mathrm{Ni}}{\mathrm{H}}=\frac{\mathrm{O}}{\mathrm{O}^{+}} \times\left(\frac{\mathrm{Ni}^{+}}{\mathrm{H}^{+}}+\frac{\mathrm{Ni}^{++}}{\mathrm{H}^{+}}\right)$.

Because we did not compute $\mathrm{Ni}^{+}$abundances, we estimated the total $\mathrm{Ni}$ abundance by applying this expression only to 


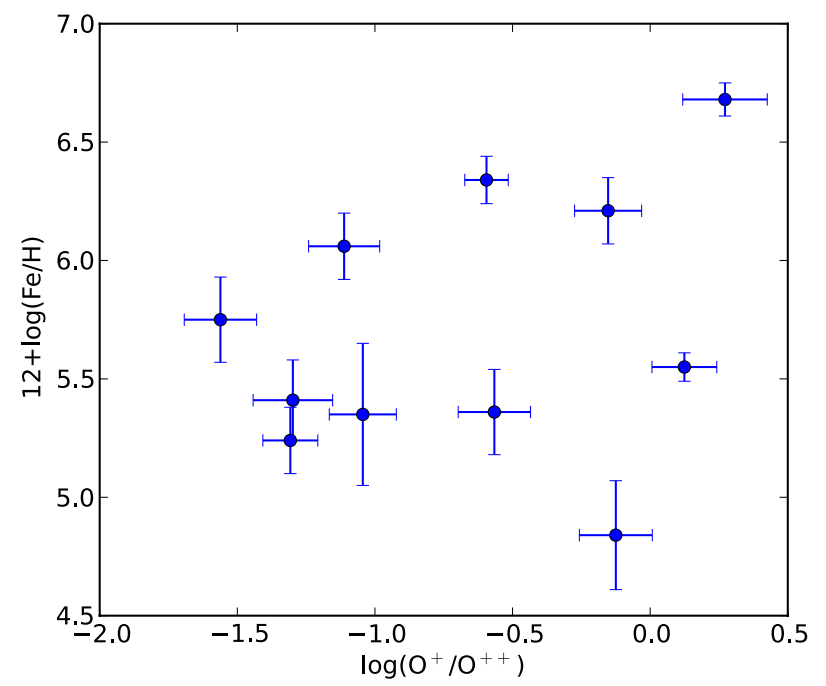

Fig. 5. Values of $\mathrm{Fe} / \mathrm{H}$ as a function of the ionization degree.

the $\mathrm{Ni}^{++}$abundance. This is a good approximation when $\log \left(\mathrm{O}^{+} / \mathrm{O}^{++}\right)<-0.1$, which is the case for Cn 1-5 and $\mathrm{He} 2-86$, but for M 1-30 and M 1-32 this expression provides only a lower limit to the real Ni abundance.

\section{Discussion of the elemental abundances}

It is a well-known fact predicted by stellar evolution models that PN central stars synthesize He in their nuclei, which is pulledup to the surface in some of the dredge-up events, thus contaminating the nebular material. The enrichment also occurs for nitrogen, although in this case, $\mathrm{N}$ is not a primary element but it is enriched via the CNO-cycle, which is much more effective in most massive progenitors. Another element that is modified in the stellar surface as a consequence of nucleosynthesis processes and dredge-up events is carbon, while at solar metallicity, oxygen, neon, and other elements are not predicted to be significantly modified (see, e.g., Marigo et al. 2003; Karakas et al. 2006, and references therein). Thus elemental abundance ratios such as N/O and $\mathrm{C} / \mathrm{O}$ reflect the occurrence of different nucleosynthesis processes in AGB stars.

In this section the total abundances obtained from ORLs or CELs are discussed. We assumed that the abundance ratios $(\mathrm{C} / \mathrm{O}$, $\mathrm{N} / \mathrm{O}, \mathrm{Ne} / \mathrm{O}, \mathrm{Ar} / \mathrm{O}, \mathrm{S} / \mathrm{O}$, etc.) are almost independent of the procedure used to calculate abundances, provided that the same types of lines (ORLs or CELs) are used for each ratio, therefore these ratios can be compared with results from other $\mathrm{PNe}$ samples and with predictions of stellar evolution models. In the following, the abundance ratios are expressed in the usual logarithmic form.

A word of caution: because our PN sample is highly biased in the sense that our objects were selected among WRPNe that showed a considerable fraction of $\mathrm{O}$ in the form of $\mathrm{O}^{++}$to find measurable O II and C II ORLs, the comparison with other more general samples needs to be made with care.

Aditionally, for two objects (namely M 1-61 and NGC 6369) the $\mathrm{N}^{+}$and $\mathrm{O}^{++}$temperatures show discrepancies that cannot be reproduced by simple photoionization models (Morisset, in prep.). The problem may be solved by using models that combine different regions that differ in their densities, in which case it is obvious that the direct method used for determining the temperature (especially that of $\mathrm{N}^{+}$) may be inadequate. These two objects are of the low-ionization class, thus the effect of a misestimated $T_{\mathrm{e}}\left(\mathrm{N}^{+}\right)$does not affect the total $\mathrm{O} / \mathrm{H}$ abundance, but it
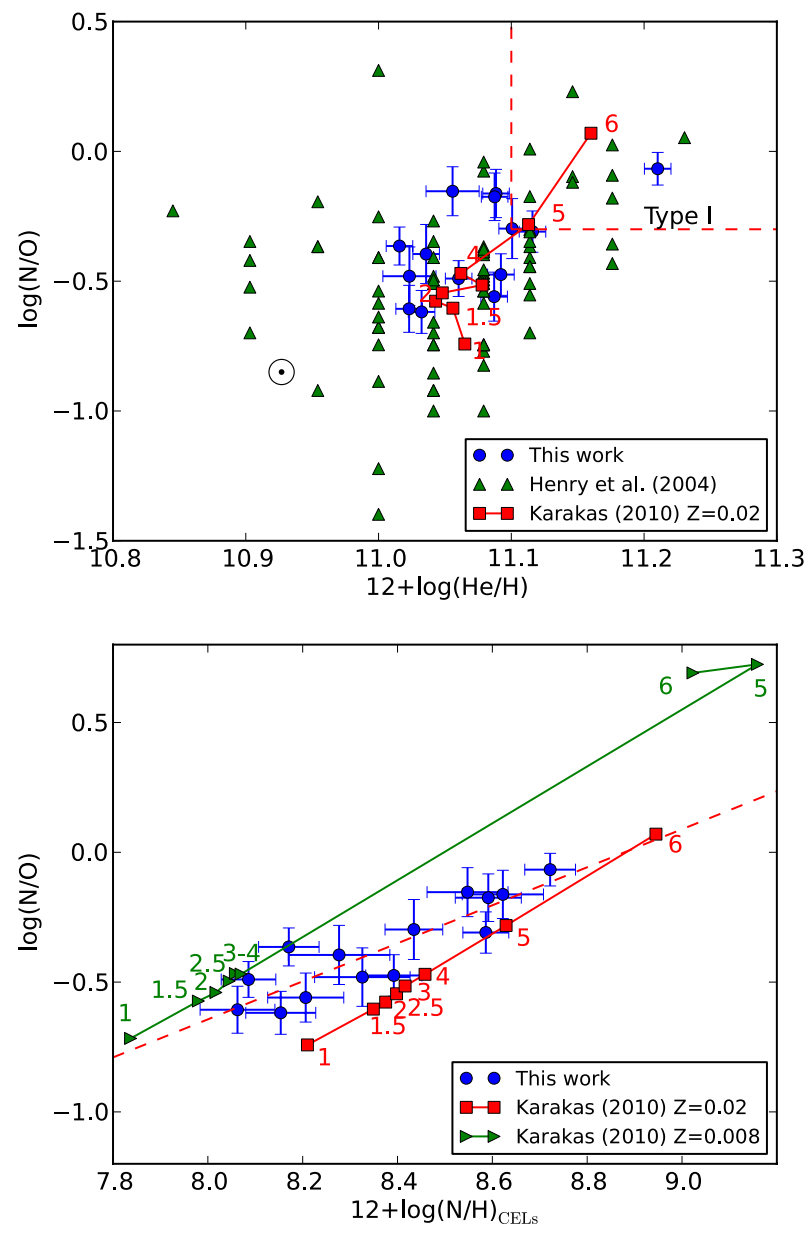

Fig. 6. Upper panel: N/O abundance ratio plotted against He abundance for our PN sample. The top-right box marks the criteria given by Peimbert (1990) to define Type I PNe. In green we included the data of the Galactic PNe sample by Henry et al. (2004). Predictions of stellar evolution models by Karakas (2010) for $Z=0.02$ and different initial masses (indicated in units of $M_{\odot}$ ) are also included. The solar symbol represents the solar values. Lower panel: relationship between $\mathrm{N} / \mathrm{O}$ abundance ratio and the total $\mathrm{N}$ abundance; the fit to our data is shown as a dashed line (see text). The predictions of stellar evolution models by Karakas (2010) for stars with different initial masses and for two different metallicities are included.

does have consequences on $\mathrm{N} / \mathrm{H}$ and $\mathrm{S} / \mathrm{H}$, which both use $\mathrm{O}^{+}$in their ICFs. To evaluate the effect, we determined abundances for the two objects using $T_{\mathrm{e}}\left(\mathrm{O}^{++}\right)$for the low-ionization zone. The $\mathrm{N} / \mathrm{H}(\mathrm{S} / \mathrm{H})$ abundances were then reduced by $0.23(0.13)$ and 0.13 (0.10) dex for M 1-61 and NGC 6369, respectively. These variations do not affect the general results presented here.

\section{1. $\mathrm{N}$ and He abundances}

Peimbert (1990) classified PNe into four groups according to their metallicity and kinematics in the Galaxy. In this classification, type I PNe present He- and N-rich envelopes $(\mathrm{He} / \mathrm{H}>0.125$ and N/O > 0.5). This classification was revised by Kingsburgh \& Barlow (1994), who classified as type I PNe whose central star suffered hot-bottom burning (HBB) and showed N/O abundance ratio higher than 0.8. In the following, we use Peimbert's classification for our objects to be consistent with previous papers of our group (Peña et al. 2013). For completeness we mention that Peimbert's type II and III PNe are nebulae that are not particularly $\mathrm{H}$ - or N-rich and have heliocentric radial velocities lower 
and higher than $60 \mathrm{~km} \mathrm{~s}^{-1}$, respectively. Type IV PNe are objects from the Galactic halo.

The upper panel of Fig. 6 shows the N/O vs. He/H distribution for our sample ( $\mathrm{N}$ and $\mathrm{O}$ derived from CELs). With green triangles we show data given for a sample of 85 Galactic PNe by Henry et al. (2004) for comparison (although care must be taken when comparing abundances obtained with different methods, different atomic parameters, etc.). Only one of our objects (Cn 1-5) is a clear type I PN and another two (M 1-30 and M 1-32) are within the limits to be considered type I PNe. Three other objects ( $\mathrm{Hb} 4, \mathrm{He} 2-86$ and NGC 5189) show a higher N/H than M 1-30 and M 1-32, but their low $\mathrm{He} / \mathrm{H}$ values exclude them from the Peimbert's type I classification. The number of PNe in our sample is small, therefore it is difficult to assess whether our sample shows a higher or similar proportion of type I PNe as the Henry et al. sample, but our three possible type I PNe (according to Peimbert's criterion) represent about 20\% against $11 \%$ (9 of 85 ) in the Henry et al. sample. Interestingly, in the Henry et al. sample $\log (\mathrm{N} / \mathrm{O})$ values cover from -1.4 to 0.4 (a similar range is found for the sample of Kingsburgh \& Barlow 1994, see their Fig. 4), while our sample shows a much more constrained $\log (\mathrm{N} / \mathrm{O})$ range, from -0.65 to 0.0 , that is, in our sample (certainly biased towards a particular type of PNe, as explained above) all objects seem richer in $\mathrm{N}$ (although not as rich as to be considered type I) than those of the Henry et al. sample. In fact, the N/O average value of our sample (excluding the three WRPNe marked as Type I) is $\langle\mathrm{N} / \mathrm{O}\rangle=0.42 \pm 0.18$, which is significantly higher than the average value of $0.31 \pm 0.14$ found for non-Type I PNe by Henry et al. (2004). This indicates that our non-Type I WRPNe are on average richer in $\mathrm{N}$ than PNe in more general samples, which may be due to a slightly higher initial mass of the progenitors (see discussion below). This is consistent with the recent result by Peña et al. (2013), showing that Galactic WRPNe are located in a disk thinner than that of average $\mathrm{PNe}$, which means that they are younger (and probably more massive) objects.

For a comparison, in the upper panel of Fig. 6 we have included the predictions of stellar evolution models by Karakas (2010) for a metallicity of $Z=0.02$. It can be seen that the progenitors of Type I PNe had initial masses larger than $5 M_{\odot}$, but also that all the PNe with $\log (\mathrm{N} / \mathrm{O})>-0.47$ (which are about half of our sample) are compatible with initial masses of the progenitors larger than $4 M_{\odot}$.

A relation between $\mathrm{N} / \mathrm{O}$ vs. $\mathrm{N} / \mathrm{H}$ is presented in the bottom panel of Fig. 6. As in other samples of PNe, a correlation is found between these quantities (see e.g., Henry 1990). A linear fit to our data gives $\log (\mathrm{N} / \mathrm{O})=0.73 \times[12+\log (\mathrm{N} / \mathrm{H})]-6.50$, with a Spearman correlation factor $r=0.86$. This correlation indicates that $\mathrm{N}$-enrichment in $\mathrm{PNe}$ occurs independently of the $\mathrm{O}$ abundance, therefore it probably is mainly a consequence of the $\mathrm{CN}$-cycle $(\mathrm{N}$ increasing at the expense of $\mathrm{C}$ ) and not of the ON-cycle. To verify this, in Fig. 7 we show the behaviour of the $\mathrm{C} / \mathrm{N}$ ratio vs. $\mathrm{N}$ abundance. In the $\mathrm{C} / \mathrm{N}$ ratio, $\mathrm{C}$ was calculated from ORLs and $\mathrm{N}$ from CELs and assuming $t^{2}>0$ (see Sect. 5.1), which would correspond to abundances derived from ORLs. A clear dependence exists, showing that $\mathrm{C} / \mathrm{N}$ decreases when $\mathrm{N}$ abundance increases up to a value $12+\log (\mathrm{N} / \mathrm{H}) \sim 8.4$. In this range, evidently $\mathrm{N}$ is increasing at the expense of $\mathrm{C}$ through the $\mathrm{CN}$-cycle (initial stellar masses lower than $3 M_{\odot}$ ). For $\mathrm{N}$ higher than this value, the tendency is less clear, $\mathrm{N}$ continues to increase but the $\mathrm{C} / \mathrm{N}$ ratio stops to decrease, indicating that $\mathrm{C}$ is being enriched through the third dredge-up process. A very interesting object (M 1-32) shows a huge C enrichment, which deserves a more detailed analysis.

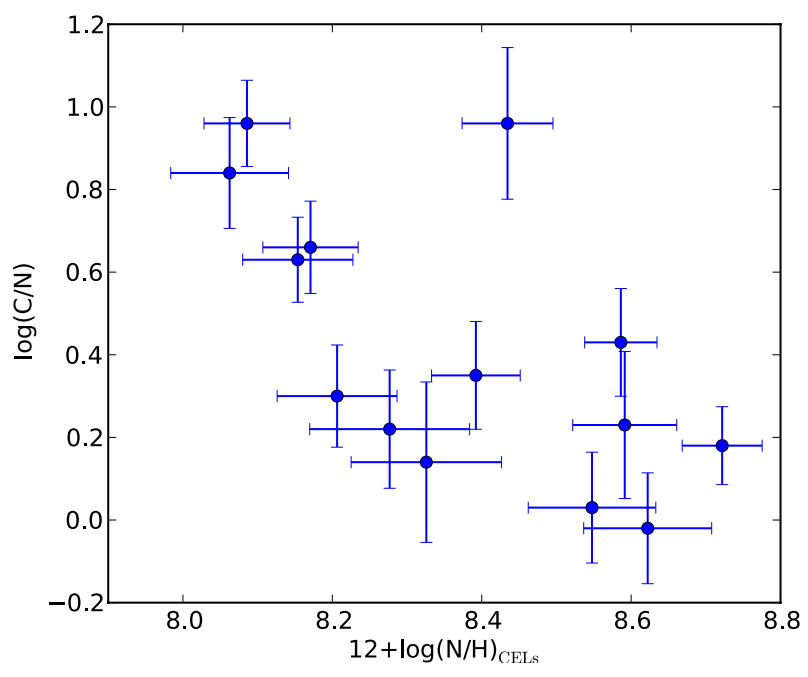

Fig. 7. $\mathrm{C} / \mathrm{N}$ abundance ratio plotted against the total $\mathrm{N}$ abundance for our sample of PNe. For the $\mathrm{C} / \mathrm{N}$ ratio, $\mathrm{C}$ was computed from ORLs and $\mathrm{N}$ from CELs and assuming a $t^{2}>0$ (see text). The total $\mathrm{N}$ abundances shown in the $x$-axis are computed from CELs and $t^{2}=0$.

In Fig. 6 (bottom) we have included the prediction of stellar evolution models for stars with initial masses between 1 and $6 M_{\odot}$ and metallicities $Z=0.02$ and $Z=0.008$ obtained from Karakas (2010). Evidently, our objects are between both sets of models. If $\mathrm{N}$ abundances were derived from ORLs, the points would move to the right by about $0.3 \mathrm{dex}(0.5 \mathrm{dex}$ in the extreme cases) and would be more in line with models of $Z=0.020$. Another interesting observation is that the linear fit to our points is less steep than models at a given metallicity. At low N/H our objects are closer to the models with $Z=0.008$ and at $12+\log (\mathrm{N} / \mathrm{H})>8.4$ they are closer to models with $Z=0.02$. This indicates that the objects with low $12+\log (\mathrm{N} / \mathrm{H})$ are probably older and started with a lower metallicity. By comparing the observed data with the models, an initial mass can be assigned to the objects. We find that our WRPNe have central stars with initial masses between about 1.5 and more than 4 solar masses, where $\mathrm{Cn} 1-5$ (the richest in N) is the most massive. Other $\mathrm{PNe}$ with initial masses larger than $4 M_{\odot}$ (those with $12+\log (\mathrm{N} / \mathrm{H}) \geq 8.44$ ) could be He 2-86, NGC 5189, Hb 4, M 1-30, and M 1-32.

\section{2. $\mathrm{C} / \mathrm{O}$ ratios and infrared dust features}

As said above, $\mathrm{C} / \mathrm{O}$ abundance ratios reflect the occurrence of different nucleosynthesis processes in AGB stars. Theoretical models predict enhanced carbon abundances in the surface of stars with initial masses above $\sim 2 M_{\odot}$ as a consequence of the third dredge-up, whereas they will be decreased in stars with masses above $\sim 4-5 M_{\odot}$ due to the HBB process (see models represented in Fig. 8). Therefore, we expect that PNe coming from progenitors with $M \lesssim 1.5 M_{\odot}$ or $M \gtrsim 4 M_{\odot}$ will have $\mathrm{C} / \mathrm{O}<1$, while those coming from stars with masses within $1.5 M_{\odot}$ and $\sim 4 M_{\odot}$ will show $\mathrm{C} / \mathrm{O}>1$.

Using the values of $\mathrm{O} / \mathrm{H}$ and $\mathrm{C} / \mathrm{H}$ calculated from ORLs from Table 12, we obtain that half of our sample is C-rich $(\mathrm{C} / \mathrm{O}>1)$, whereas the other half is $\mathrm{O}$-rich $(\mathrm{C} / \mathrm{O}<1)$. This is not unusual; in the sample analysed by Kingsburgh \& Barlow (1994) $40 \%$ of the PNe were C-rich, and Rola \& Stasińska (1994), using data from the literature, found that $35 \%$ of their sample of $85 \mathrm{PNe}$ were $\mathrm{C}$-rich (in both cases $\mathrm{C}$ and $\mathrm{O}$ were determined via CELs). In 


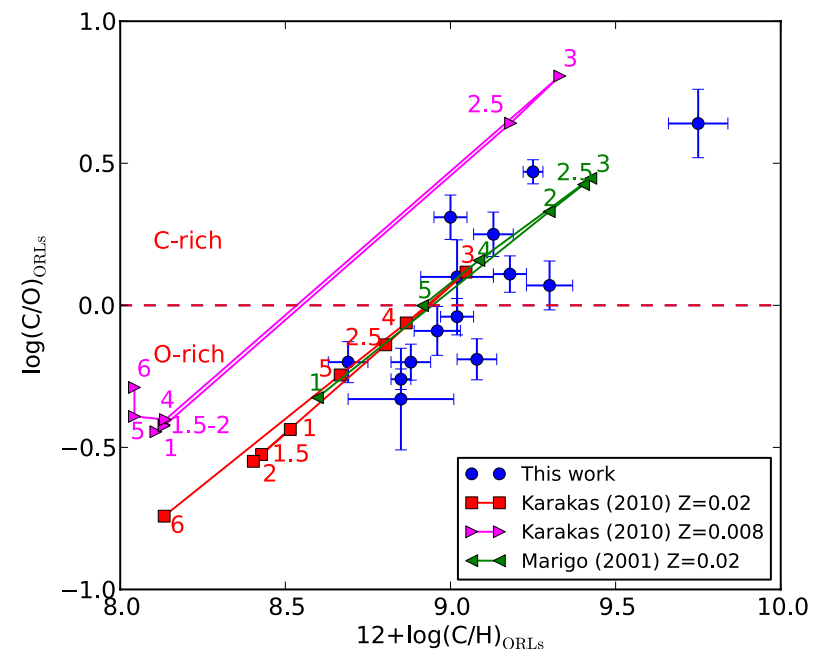

Fig. 8. $\mathrm{C} / \mathrm{O}$ abundance ratio plotted against $\mathrm{C}$ abundance for our sample of PNe. The horizontal dashed line represents the separation between $\mathrm{C}$-rich and O-rich objects. The predictions of stellar evolution models by Karakas (2010) (red models: $Z=0.02$, magenta models: $Z=0.008$ ) and by Marigo (2001) (green models: $Z=0.02$ ) for stars with different initial masses (indicated in units of $M_{\odot}$ ) are included.

our sample the fraction of C-rich nebulae is slightly larger than in these two samples.

Figure 8 shows the $\mathrm{C} / \mathrm{O}$ values as a function of $\mathrm{C} / \mathrm{H}$ for our $\mathrm{PNe}$, all of them calculated from ORLs. A clear correlation exists, reflecting the effect of the third dredge-up, which pulls out freshly produced carbon to the stellar surface. In this figure we overplotted the predictions from theoretical models calculated by Karakas (2010) with metallicities $Z=0.020$ and 0.008 , and Marigo (2001) with metallicity $Z=0.020$, for different initial masses. Models show the behaviour described above in the sense that $\mathrm{C}$ abundance and $\mathrm{C} / \mathrm{O}$ ratio increase for masses increasing from 1 to about $3 M_{\odot}$ and then decrease for larger masses.

We discuss our objects compared with models from Marigo (2001), which better reproduce our data. Apparently, our C-rich objects $(\mathrm{C} / \mathrm{O}>1)$ were produced by stars with initial masses between 1.5 and about $5 M_{\odot}$. Among them we find Cn 1-5, M 1-30 and NGC 5189 (with initial masses near or above $4 M_{\odot}$ as derived from their $\mathrm{N}$ abundances) and NGC 2867, NGC 6369, and Pe 1-1, which probably descend from stars with masses between 1.5 and $2.5 M_{\odot}$. The case of M 1-32, which is the PN richest in $\mathrm{C}$ in our sample, is difficult to understand because there is no model that reproduces its extremely large $\mathrm{C}$ abundance. Its progenitor may have had an initial mass around $3 M_{\odot}$, for which models predict the highest $\mathrm{C}$ enrichment, and for some reason, the third dredge-up was very efficient in this object.

It is difficult to assign an initial stellar mass to the objects with $\mathrm{C} / \mathrm{O}<1$ because the model curve is bi-valued in this zone. Among these objects we find $\mathrm{Hb} 4$ and $\mathrm{He} 2-86$, which in the $\mathrm{N}$-diagram appear as objects coming from stars with an initial mass larger than $4 M_{\odot}$, hence they probably suffered HBB, loosing $\mathrm{C}$ in the process. They are not classified as Peimbert type I due to their low $\mathrm{He} / \mathrm{H}$ abundance (perhaps this criterion should be revised). The rest (five $\mathrm{PNe}$ with $\mathrm{C} / \mathrm{O}<1$ ) are expected to have descended from stars with initial masses between 1 and $1.5 M_{\odot}$.

Summarizing, from $\mathrm{N}$ and $\mathrm{C}$ abundances and their ratios with $\mathrm{O}$, we find consistent results with regard to the initial stellar masses of the progenitors. About half of our objects had a progenitor with an initial mass around or larger than four solar masses.

Another aspect of $\mathrm{C} / \mathrm{O}$ abundance ratio is that its value in the atmospheres of AGB stars determines the composition of the grains that are formed. In principle, we expect carbon-based grains (such as PAHs or $\mathrm{SiC}$ ) in $\mathrm{PNe}$ with $\mathrm{C} / \mathrm{O}>1$ and oxygenbased grains (mainly silicates) in $\mathrm{PNe}$ with $\mathrm{C} / \mathrm{O}<1$ (see, e.g., Whittet 2003). Therefore a correlation between $\mathrm{C} / \mathrm{O}$ and dust features is expected. We compiled all available information for our sample of PNe from the literature and also inspected the unpublished Spitzer spectra for NGC 2867 and PB 8. As shown in Table 13, the PNe can be divided into two categories. The first one contains two C-rich PNe showing only C-rich features: NGC 6369 with PAHs and SiC, and Pe 1-1 with PAHs and the broad feature at $30 \mu \mathrm{m}$ that is usually associated with $\mathrm{MgS}$ (Cohen \& Barlow 2005; Stanghellini et al. 2012). The second group corresponds to $\mathrm{PNe}$ with $\mathrm{C} / \mathrm{O}$ either below or above one and a mixed chemistry of dust grains, that is, where crystalline silicates and PAHs are simultaneously found in the spectra. The explanation for this may be different in C-rich and O-rich environments. The preferred scenario for the $\mathrm{PNe}$ with $\mathrm{C} / \mathrm{O}>1$, Cn 1-5, M 1-32, and NGC 2867, is that the silicates were formed when the star was still O-rich, whereas the PAHs formed more recently, after the third dredge-up transformed the O-rich star to a C-rich star (Waters et al. 1998). For the O-rich $(\mathrm{C} / \mathrm{O}<1)$ PNe, Guzmán-Ramírez et al. (2011) suggested that PAHs may form in the dense torus. Alternatively, PAH emission may come from the surrounding ISM. The other three nebulae are M 1-30, PC 14, and NGC 5189, for which we do not have information of the type of grains present.

\section{3. $\alpha$-elements}

Oxygen, neon, sulphur, argon, and chlorine are $\alpha$-elements that, in principle, are only synthesized in massive stars, hence they are not affected by nucleosynthesis processes in the PNe progenitor stars (Marigo 2001).

In Fig. 9 we present the abundance ratios of all $\alpha$-elements (all except $\mathrm{Mg}$, which has only been detected in four objects and whose abundance is quite uncertain) over $\mathrm{O}$, plotted againts the total $\mathrm{O} / \mathrm{H}$ ratio derived from CELs. We overplotted solar abundances from the most recent compilation by Lodders (2010).

In Sect. 2.2 we computed the abundances for the high IP ions (IP > $39 \mathrm{eV}$ ) using two sets of physical conditions: the commonly used $T_{\mathrm{e}}([\mathrm{O} \mathrm{III}])$ (set A), and the rarely used $T_{\mathrm{e}}$ ([Ar IV]) (set B). In Fig. 9 we represented the abundances calculated using set $\mathrm{A}$ as red circles, and we have connected these points with the black stars, which are the points for which we have considered set B. With this comparison, we aim to verify the reliability of using $T_{\mathrm{e}}$ ([Ar IV]) as representative of a high-ionization zone in a scheme of three zones. As we can see in Fig. 9, the effect is only strong for $\mathrm{Ne}$, because $\mathrm{Ne}^{++}$is the dominant ionization stage for $\mathrm{Ne}$ in the ionization degree range of our sample. Moreover, by assuming set $\mathrm{B}$ as representative of the high-ionization zone, the spread of $\mathrm{Ne} / \mathrm{O}$ ratios is very wide and makes almost half of the sample Ne-poor objects, which cannot be explained by any stellar evolution model. The results obtained with set A are much more reasonable and agree with stellar evolution models, which predict that the $\mathrm{Ne} / \mathrm{O}$ ratio probably does not vary, because both elements are produced by stars in the same mass range. An explanation for this behaviour would be that the ratio between collisional strengths for [Ar IV] $\lambda \lambda 4711+40$ and $\lambda \lambda 7170+262$ lines is not reliable. We carried out some tests by increasing the collisional stregths of the $\lambda \lambda 4711+40$ transitions by a factor 
Table 13. $\mathrm{C} / \mathrm{O}$ values and infrared dust features.

\begin{tabular}{lccc}
\hline \hline & $\mathrm{SiC} / 30 \mu \mathrm{m}+\mathrm{PAHs}$ & PAHs + crystalline silicates & No information \\
\hline $\mathrm{C} / \mathrm{O}>1$ & NGC 6369, Pe 1-1 & Cn 1-5, M 1-32, NGC 2867 & M 1-30, NGC 5189 \\
$\mathrm{C} / \mathrm{O}<1$ & - & Hb 4, He 2-86, M 1-25, & PC 14 \\
& & M 1-61, M 3-15, PB 8 & \\
\hline
\end{tabular}

Notes. Dust identifications from Cohen \& Barlow (2005), Perea-Calderón et al. (2009), and Stanghellini et al. (2012).
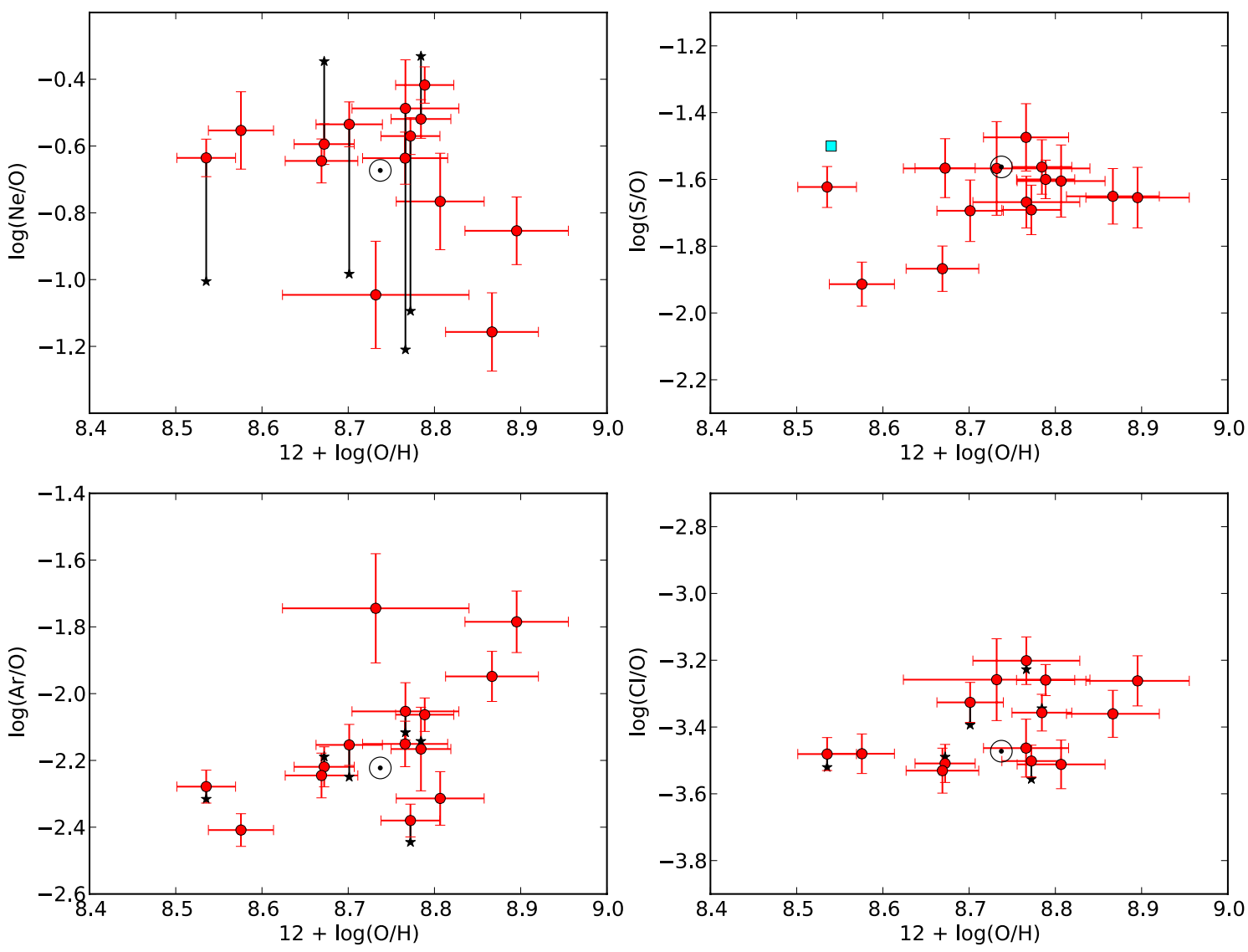

Fig. 9. $\alpha$-element/O abundance ratios for our sample of PNe. Stars indicate abundances computed using $T_{\mathrm{e}}$ ([Ar IV]) for ions with IP $>39 \mathrm{eV}$, while red circles represent abundances computed using $T_{\mathrm{e}}([\mathrm{O} \mathrm{III}])$ (see discussion in text). The solar symbol indicates the solar abundances taken from the compilation by Lodders (2010). The blue square in the $\log (\mathrm{S} / \mathrm{O})$ plot is the value obtained for the Orion nebula from CELs. The $y$-axis ranges over 1.2 dex.

of 1.5 , which led to a lower $T_{\mathrm{e}}$ ([Ar IV]); but the problem was not solved, as the abundance of Ne decreased in some objects, but it increased significantly in others. In addition, there are uncertainty sources in the determination of the total $\mathrm{Ne}$ abundance due to the used ICF (see below).

Despite their relatively large dispersion, the $\mathrm{Ar} / \mathrm{O}$ and $\mathrm{Cl} / \mathrm{O}$ ratios seem to be consistent with what is expected for $\alpha$-elements, in the sense that these elements evolve in lockstep with O. However, interestingly, in Fig. 9 there are three objects that appear to be Ar-rich and Ne-poor at the same time. These objects are M 1-25, M 1-30, and M 1-32 and are the objects with the lowest ionization degree in our sample. In Fig. 10 we can see this behaviour. The problem with $\mathrm{Ne}$ is mainly due to the well-known fact that the classical ICF scheme for $\mathrm{Ne}$ underestimate the total $\mathrm{Ne}$ abundance for low ionization nebulae (Torres-Peimbert \& Peimbert 1977; Peimbert et al. 1992) because a considerable fraction of $\mathrm{Ne}^{+}$coexists with $\mathrm{O}^{++}$. Additionally, photoionization models have proven that this ICF underestimates the total $\mathrm{Ne}$ for $\mathrm{PNe}$ with low $T_{\text {eff }}$
(Delgado-Inglada et al., in prep.). On the other hand, the ICF(Ar) from Kingsburgh \& Barlow (1994) seems to overestimate the Ar abundance for the same three objects. This has been proven by recent photoionization models, which predict that with these ICFs the Ar abundance could be overestimated by between 0.2 and 0.7 dex, depending on the exact ICF scheme and on the assumed central star temperature, being less overestimated if the $T_{\text {eff }}$ of the central star is relatively low (about $50000 \mathrm{~K}$, DelgadoInglada et al., in prep.), which is the case for M 1-25 and M 1-32. A drop in the Ar abundance by about $0.2-0.3$ dex for these objects would reconcile their abundances with what is expected for an $\alpha$-element (see Fig. 9).

In Fig. 9 (upper right), the behaviour of S/O vs. O abundance for our sample is shown. We included the solar value $(-1.57$ Lodders 2010) and the Orion nebula value (blue square, $\log (\mathrm{S} / \mathrm{O})=-1.5$, from CELs, Esteban et al. 2004; García-Rojas \& Esteban 2007). In general, the PNe sulfur abundances are lower. This anomaly in the sulfur abundance in PNe is a wellknown problem that has been discussed frequently in the past 


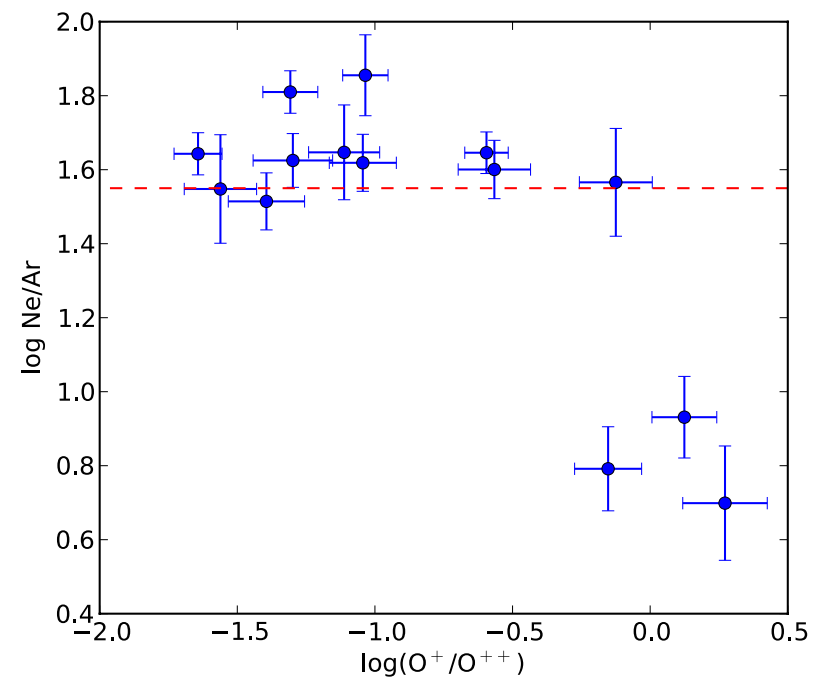

Fig. 10. Ne/Ar abundance ratio vs. the ionization degree. The dashed red line represents the solar $\mathrm{Ne} / \mathrm{Ar}$ abundance ratio. $\mathrm{Ne} / \mathrm{Ar}$ is clearly underestimated for three objects.

years (e.g. Henry et al. 2004, 2012; Shingles \& Karakas 2013) and, so far, it is an unresolved question. Several explanations have been proposed, all of them revised by Henry et al. (2012): a) incorrect ICFs that do not properly take into account the highly ionized $\mathrm{S}^{+3}$ state; b) sulfur gas phase depletion due to dust or molecule formation; c) effects of dielectronic recombination in the sulfur ionization balance, and, very recently; d) the possibility that $\mathrm{S}$ might be destroyed by nucleosynthetic processes in low- and intermediate-mass stars during stellar evolution, which has been studied in depth by Shingles \& Karakas (2013). Most of these explanations have failed to explain the anomaly, which is still a subject of debate in PN physics.

For our objects we can analyse the possibility of sulfur atoms to be partially deposited in C-rich dust grains (Whittet 2003). The lowest sulfur abundances in our sample are found in Pe 1-1, NGC 6369, and NGC 2867. The first two PNe show C-rich features (PAHs, $\mathrm{SiC}$, and/or the broad feature at $30 \mu \mathrm{m}$ ) in their infrared spectra and therefore their low gaseous sulfur abundance might be partially due to the depletion of sulfur atoms onto dust grains. NGC 2867 does not show C-rich features in its spectrum, but the $\mathrm{C} / \mathrm{O}$ value above one might also indicate some depletion of sulfur atoms.

However, Henry et al. (2012) ruled out this scenario of sulfur depletion in dust to explain the sulfur anomaly in PNe. These authors proposed that the most probable explanation is that ICFs are not properly taking into account the contribution of $\mathrm{S}$ ionization stages higher than $\mathrm{S}^{++}$. Using photoionization models, Rodríguez \& Delgado-Inglada (2011) found that the ICF derived by Stasińska (1978) introduces a bias in the computed sulfur abundances that can be significant even for low-ionization PNe, which would partially explain the situation.

\section{The abundance discrepancy}

As discussed in Sect. 1, the discrepancy between ionic abundances of several ions obtained from ORLs and CELs is a wellknown problem that has dozens of references in the literature, both in PNe and H II regions (see e.g. García-Rojas \& Esteban 2007; Esteban et al. 2009; McNabb et al. 2013; Fang \& Liu 2013, and references therein). The physical explanation for this discrepancy is still unclear, and several scenarios have been

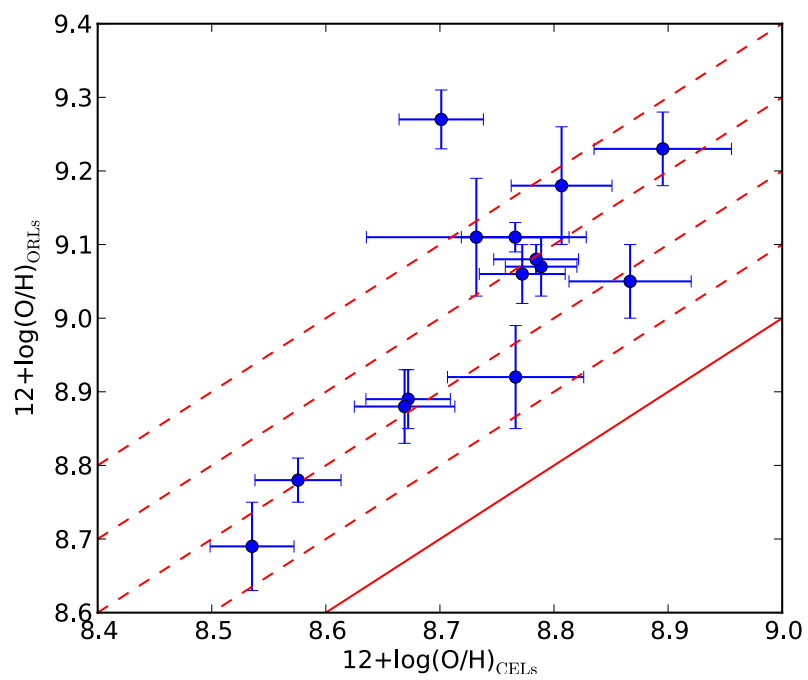

Fig. 11. $\mathrm{O} / \mathrm{H}$ derived from $\mathrm{ORLs}$ plotted against $\mathrm{O} / \mathrm{H}$ derived from CELs. The continous line represents the equality. Dashed lines represent ADFs of $0.1,0.2,0.3$, and 0.4 dex. The $\mathrm{O} / \mathrm{H}$ derived from ORLs is about a factor of 2-3 higher than that derived from CELs.

proposed; but, most references are focused on two scenarios: spatial temperature fluctuations in the ionized gas, and a cold, metal-rich ionized gas component embedded in the ambient gas.

Torres-Peimbert et al. (1980) proposed for the first time that the the cause of the discrepancy between abundance computed from CELs and ORLs might be spatial temperature fluctuations (parametrized by $t^{2}$ ). Several mechanisms have been proposed to explain temperature fluctuations in $\mathrm{H}$ II regions and/or PNe (see Esteban 2002; Peimbert \& Peimbert 2006, and references therein) but to date, it is still unclear which mechanisms could produce them.

On the other hand, Liu et al. (2000) proposed that a twocomponent nebular model with H-deficient (metal-rich) inclusions embedded in the normal metallicity gas, can account for most of the observed patterns. This behaviour has been probed for two born-again PNe: Abell 30 (Wesson et al. 2003) and Abell 58 (Wesson et al. 2008) and, probably for the PN Hf 2-2 (Liu et al. 2006), which presents the largest $\operatorname{ADF}\left(\mathrm{O}^{++}\right)$found until now. However, the origin of these metal-rich inclusions in $\mathrm{PNe}$ is still a matter of discussion in the astronomical community (see Henney \& Stasińska 2010, for an extensive review of this question). A similar model has been proposed for $\mathrm{H}$ II regions by Tsamis \& Péquignot (2005) and a possible origin of these inclusions in these nebulae has been proposed by Stasińska et al. (2007). Therefore, the last word about this question has not been written yet.

Alternatively to these two scenarios, or complementary to them, Nicholls et al. (2012) have proposed that departures of the Maxwell-Boltzmann equilibrium energy distribution of the electrons in the gas in the form of a $\kappa$-distribution of the thermal electrons, might be a potential explanation for the observed electron temperatures and chemical abundance behaviour in $\mathrm{H}$ II regions and PNe. Additionally, very recent studies have proposed that high-density objects, such as proplyds, and high-velocity flows, such as Herbig-Haro objects, might play an important role in introducing uncertainties in the determination of physical conditions and chemical abundances using CELs (Mesa-Delgado et al. 2009a, 2012; Núñez-Díaz et al. 2012; Tsamis et al. 2011).

In Table 12, two sets of abundances (from CELs and from ORLs) are presented for O, which so far is the element with the 

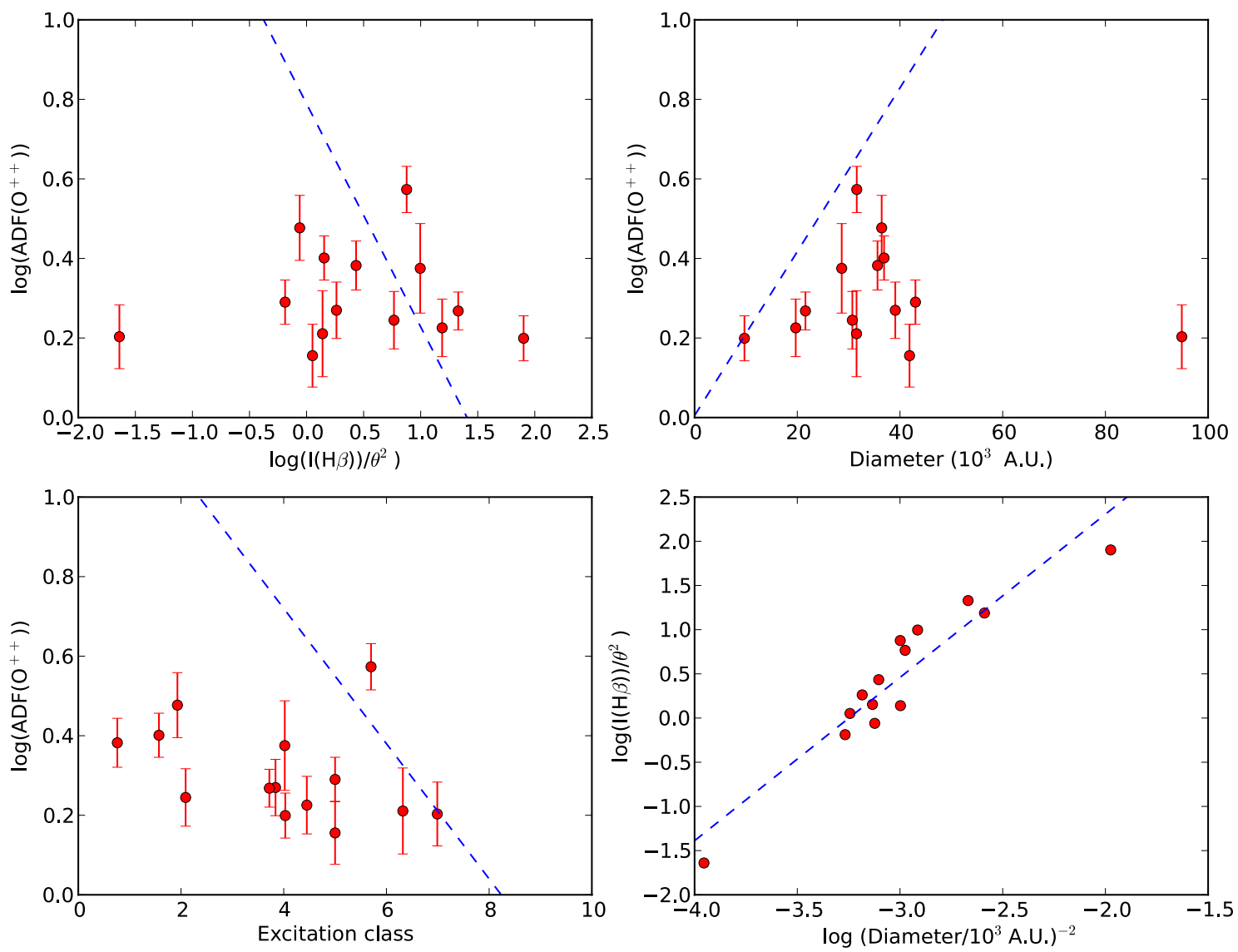

Fig. 12. $\mathrm{ADF}\left(\mathrm{O}^{++}\right)$plotted against mean nebular surface brightness (upper left panel); absolute nebular diameters (upper right panel) and excitation class as defined by Dopita \& Meatheringham (1990) (lower left panel). The dashed lines represent the fits obtained by Liu et al. (2004) for a sample of $19 \mathrm{PNe}$. In the lower right panel we show the relation between the nebular surface brightness and the nebular diameter, which are clearly correlated; the line is a fit to the data.

best-determined abundance. In Fig. 11 we plot the $\mathrm{O} / \mathrm{H}$ derived from ORLs vs. $\mathrm{O} / \mathrm{H}$ derived from CELs. When CELs are used, we obtain $12+\log (\mathrm{O} / \mathrm{H})$ abundances ranging from about 8.5 (NGC 6369) to about 8.90 (M 1-30). When ORLs are used, the $12+\log (\mathrm{O} / \mathrm{H})$ values range from about 8.7 (NGC 6369) to more than 9.2 (Hb 4 and $\mathrm{M} 1-30)$. In general, we found that $\mathrm{O} / \mathrm{H}$ derived from ORLs is higher than $\mathrm{O} / \mathrm{H}$ derived from CELs by factors between 1 and 3. In neither case did we find a large ADF (larger than 5), as has been found in several Galactic PNe (see e.g. McNabb et al. 2013). Therefore, it seems that the [WC] nature of the central star does not particularly affect the ADFs in these nebulae.

At this point we should consider that the solar $12+\log (\mathrm{O} / \mathrm{H})$ value currently accepted is 8.73 (Lodders 2010) ${ }^{4}$ and the same value for the gas+dust Orion nebula (showing the present ISM abundances) based on CELs/ORLs is 8.59/8.73 (Esteban et al. 2004). Progenitors of PNe have ages between 1 and $10 \mathrm{Gyr}$, and it is expected that their initial $\mathrm{O}$ abundances were similar or lower than in the Sun, but not higher. Therefore the $\mathrm{O} / \mathrm{H}$ abundance ratios derived from ORLs are difficult to reconcile with this view, unless the central stars have modified the $\mathrm{O}$ in their

\footnotetext{
4 The most widely used reference for solar abundances is that of Asplund et al. (2009), but the Lodders compilation includes updated oxygen abundances and a detailed discussion of the evolution of oxygen abundance determinations in the Sun; aditionally, meteoritic and photosferic abundances are given and a value is recommended; given the similarity within the uncertainties between the values published by both authors, we have preferred to cite Lodders's work.
}

surfaces as a consequence of nucleosynthesis. Although some evolution models of LIMS (low to intermediate mass stars) predict such a phenomenon, it occurs at metallicities much lower than in the Milky Way (Marigo et al. 2003; Karakas \& Lattanzio 2003). On the other hand, Lodders (2003) claimed that solar photospheric abundances are not representative of the protoSun, because heavy-element fractionation has altered the photospheric abundances by about -0.074 dex in the Sun's lifetime. However, this effect is still too low to explain the large differences ( $\sim 0.3$ dex in average) found between $\mathrm{O} / \mathrm{H}$ values derived from ORLs in PNe and those predicted by chemical evolution models. In the following we adopted the abundances given by ORLs (or considering a $t^{2}$ parameter higher than 0.00) as representative of the nebulae, but we keep in mind the inconsistency reported here.

In an extensive study of the abundance discrepancy in several $\mathrm{PNe}$, Liu et al. (2004) found correlations between $\operatorname{ADF}\left(\mathrm{O}^{++}\right)$ and several nebular properties, such as metallicity, mean surface brightness, diameter, excitation class and density. Some of these correlations were previously found by other authors (e. g. Garnett \& Dinerstein 2001). Although our data do not cover a range of ADFs as wide as that of these authors, we consider that a comparison could be interesting. We have obtained the mean surface brightness and the angular sizes for our PNe from the Strasbourg - ESO catalogue of Galactic planetary nebulae (Acker et al. 1992); heliocentric distances to compute absolute nebular diameters were taken from Stanghellini \& Haywood (2010), and excitation classes were computed following Dopita \& Meatheringham (1990). In Fig. 12 we explore these relations 

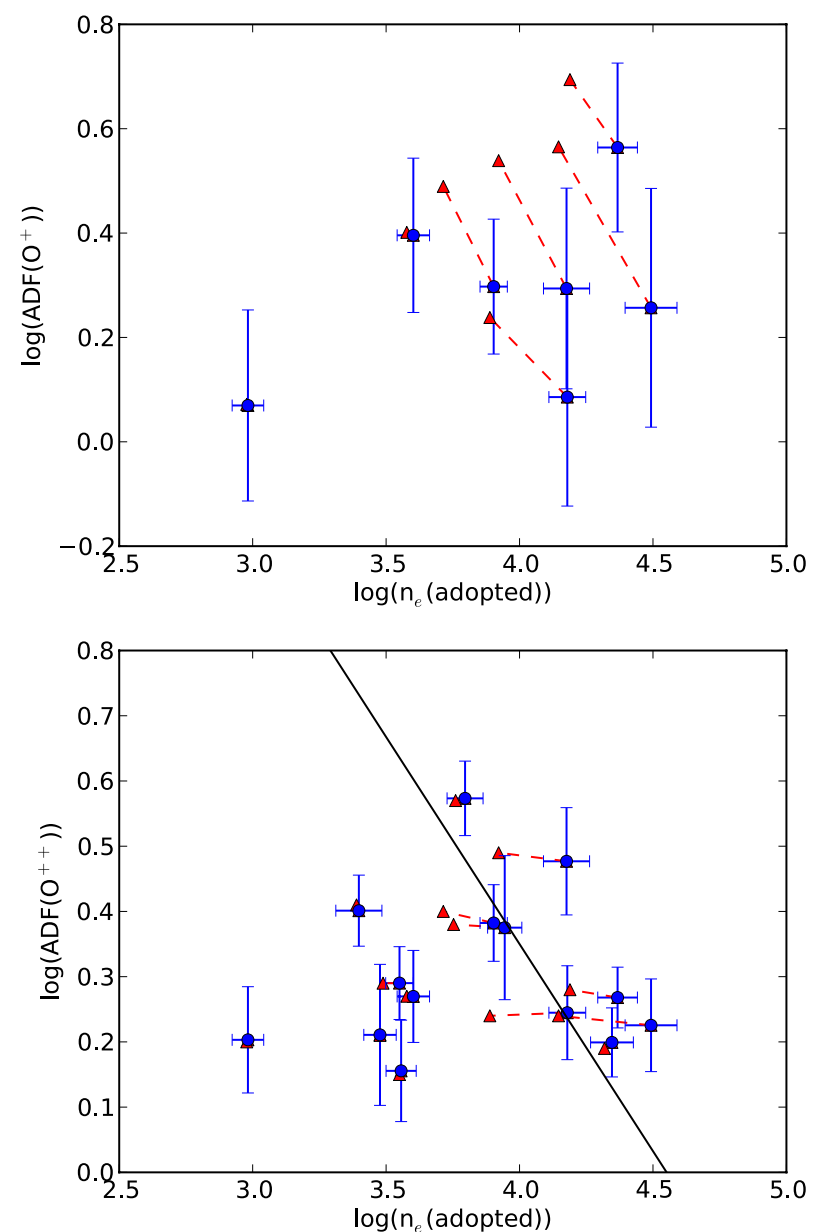

Fig. 13. $\mathrm{ADF}\left(\mathrm{O}^{+}\right)$and $\operatorname{ADF}\left(\mathrm{O}^{++}\right)$vs. $\log \left(n_{\mathrm{e}}\right)$. Blue dots represent the values assuming the densities shown in Table 5 of Paper I. Red dots represent the values obtained for $n_{\mathrm{e}}([\mathrm{S}$ II $]$ ) for all the PNe. Note that although $\operatorname{ADF}\left(\mathrm{O}^{++}\right)$remains almost unchanged, the $\operatorname{ADF}\left(\mathrm{O}^{+}\right)$is very sensitive to the assumed density. The line is the correlation found by Robertson-Tessi \& Garnett (2005).

with our data and found for the range of ADFs of our sample no clear correlation. It seems that the correlations found by Garnett \& Dinerstein (2001) and Liu et al. (2004) are not as evident if we exclude large ADFs from the sample.

In Fig. 13 we show the $\operatorname{ADF}\left(\mathrm{O}^{+}\right)$and $\operatorname{ADF}\left(\mathrm{O}^{++}\right)$plotted against the density. In the upper panel, we show that $\operatorname{ADF}\left(\mathrm{O}^{+}\right)$is extremely sensitive to the assumed density, whereas $\operatorname{ADF}\left(\mathrm{O}^{++}\right)$ is not. Hence $\operatorname{ADF}\left(\mathrm{O}^{+}\right)$should be taken with some caution because this ion comes from the outermost zones of the PNe (especially if the PN is highly excited) and might be only representative for a small volume of the ionized gas.

Finally, in Fig. 14 we show the N/O ratio plotted against the $\operatorname{ADF}\left(\mathrm{O}^{++}\right)$. We did this to determine whether the ADF depends on the enrichment pattern of the PNe. Liu et al. (2004) found a slight correlation between $\mathrm{N} / \mathrm{O}$ and the $\operatorname{ADF}\left(\mathrm{O}^{++}\right)$although with a large scatter. In our case, the dispersion of the data is large and no apparent correlation is found.

\subsection{Determination of $t^{2}$}

Given the different dependence on the electron temperature of the CELs and ORLs emissivities, and assuming the validity of the temperature fluctuations paradigm, we can estimate the mean square temperature fluctuations parameter, $t^{2}$, from the

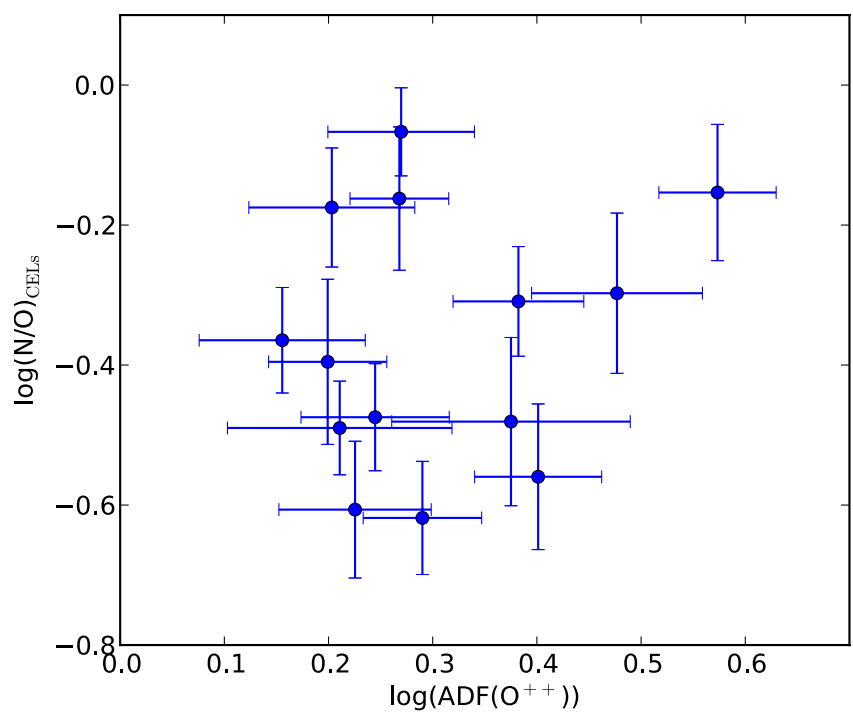

Fig. 14. $\mathrm{N} / \mathrm{O}$ vs. $\operatorname{ADF}\left(\mathrm{O}^{++}\right)$.

comparison between abundances derived from both kind of lines for a given ion (see Peimbert et al. 2004, and references therein for a detailed description of temperature fluctuations formalism). In particular, we estimated the values of the $t^{2}$ parameter from the ADFs obtained for $\mathrm{O}^{+}, \mathrm{O}^{++}, \mathrm{C}^{++}$(only for two objects) and $\mathrm{Ne}^{++}$. The values obtained from the comparison of $\mathrm{C}^{++}$ionic abundances using CELs and ORLs are quite uncertain because the abundances from CELs were obtained from IUE UV observations, where aperture and extinction effects introduce large uncertainties. The values we computed for our objects are moderate and quite consistent among each other within the uncertainties, and in principle, they might be caused by spatial temperature fluctuations in the observed volume of gas.

Following Peimbert et al. (2002), we also computed the $t^{2}$ obtained from the application of a maximum-likelihood method (MLM) to search for the physical conditions, including $\mathrm{He}^{+} / \mathrm{H}^{+}$ ratios and optical depths, that simultaneously fit all the measured lines of He I (see Sect. 2.1).

Additionally, from the comparison between electron temperatures obtained from CELs and from the hydrogen recombination continuum discontinuities, we can obtain an indication of $t^{2}$ (see Torres-Peimbert et al. 1980; García-Rojas et al. 2009, and references therein). Unfortunately, the measurement of Balmer and Paschen discontinuities in our spectra was rather difficult and, therefore, these estimates were unreliable (see Paper I).

In Fig. 15 we show the comparison between $t^{2}\left(\mathrm{O}^{++}\right)$and $t^{2}\left(\mathrm{He}^{+}\right)$(upper panel) and $t^{2}\left(\mathrm{O}^{++}\right)$against $t^{2}\left(\mathrm{O}^{+}\right)$(lower panel). Clearly, there is an overall agreement between $t^{2}\left(\mathrm{He}^{+}\right)$and $t^{2}\left(\mathrm{O}^{++}\right)$, but this agreement is not evident in the second case. It is important to mark that $\mathrm{He}^{+}$and $\mathrm{O}^{++}$coexist in most of the volume for $\mathrm{PNe}$ with excitation classes similar to those of our sample, but $\mathrm{O}^{+}$and $\mathrm{O}^{++}$represent two distinct zones in each $\mathrm{PN}$. However, given the large uncertainties in the determination of $t^{2}\left(\mathrm{O}^{+}\right)$, we can say that most of the $t^{2}$ values agree within the uncertainties for our sample, with some exceptions that do not affect to the determination of total abundances for $t^{2}>0.00$ (see next paragraph).

In Fig. 16 we plot the $t^{2}\left(\mathrm{O}^{+}\right) / t^{2}\left(\mathrm{O}^{++}\right)$ratio against $\log \left(\mathrm{O}^{+} / \mathrm{O}\right)$, and found a clear anti-correlation. We ran a generalized Kendall $\tau$-correlation test on these data and found $\tau=-1.2$, and a probability that both quantities are not correlated of $\sim 14 \%$, hence, they are probably correlated. However, this test should be taken with 

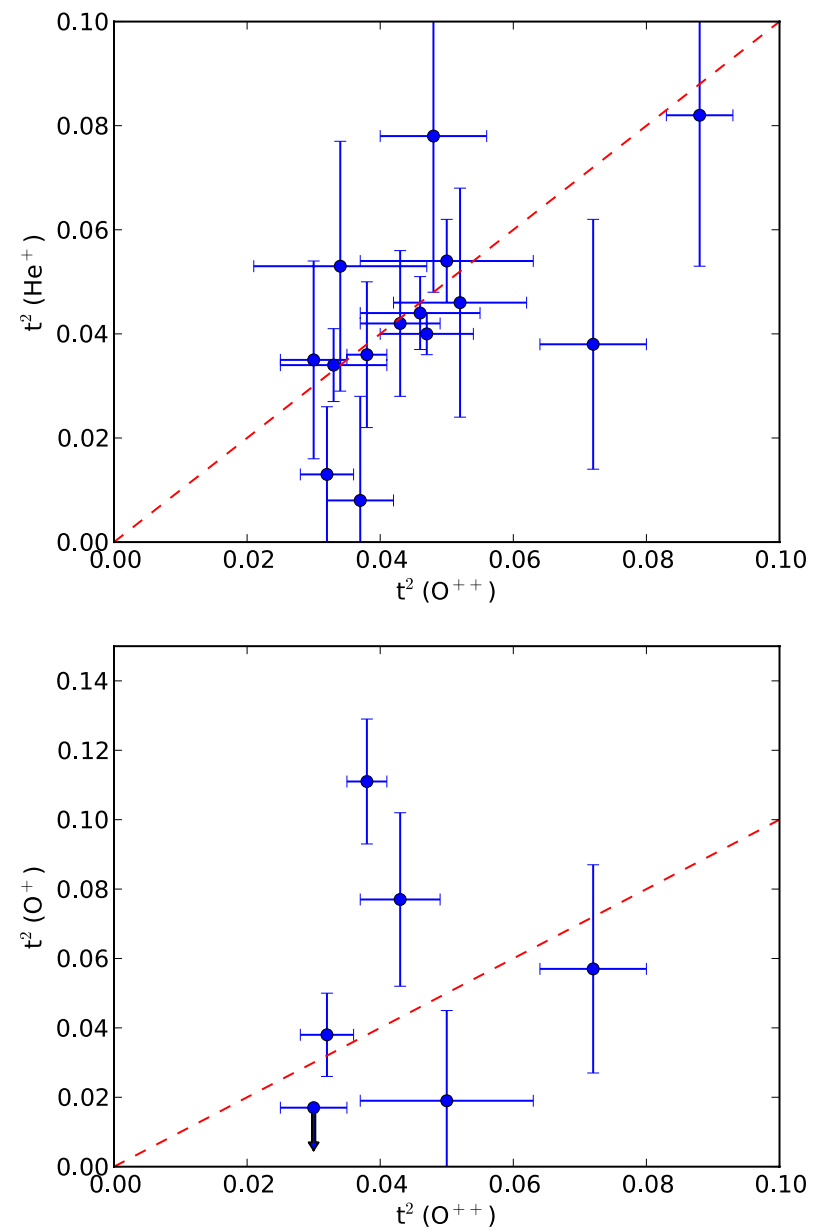

Fig. 15. $t^{2}\left(\mathrm{O}^{++}\right)$against $t^{2}\left(\mathrm{He}^{+}\right)$(upper panel) and against $t^{2}\left(\mathrm{O}^{+}\right)$(lower panel); lines represent equality. Clearly, there is an overall agreement between $t^{2}\left(\mathrm{He}^{+}\right)$and $t^{2}\left(\mathrm{O}^{++}\right)$. The agreement between the $t^{2}\left(\mathrm{O}^{+}\right)$and $t^{2}\left(\mathrm{O}^{++}\right)$values is poorer (see text).

caution when the data set is small, as in our case. Undoubtedly, more high-quality observations of multiplet $1 \mathrm{O}$ I ORLs are needed to minimize the $t^{2}\left(\mathrm{O}^{+}\right)$uncertainties and confirm (or discard) this tendency. However, if this anti-correlation is real, there must be one (or a set of) physical mechanism to explain why temperature fluctuations in the $\mathrm{O}^{+}$zone become higher than in the $\mathrm{O}^{++}$zone when the relative $\mathrm{O}^{+}$volume is smaller. Nevertheless, given the relatively low importance of $\mathrm{O}^{+}$in these cases, the total $\mathrm{O}$ abundance for $t^{2}>0.00$ is not affected.

To compute the final $t^{2}$ parameter we averaged the values obtained from the ADFs of $\mathrm{O}^{+}$and $\mathrm{O}^{++}$and the $t^{2}\left(\mathrm{He}^{+}\right)$. We weighted the $t^{2}$ of $\mathrm{O}^{+}$and $\mathrm{O}^{++}$with the relative abundance of each ion. We discarded the values derived from the ADFs of $\mathrm{Ne}^{++}$because the $\mathrm{Ne}$ II ORLs are very weak, and those derived from the $\mathrm{ADF}$ of $\mathrm{C}^{++}$for the reasons discussed above. In Table 14 we show all computed $t^{2}$. Following the formulae given by Peimbert (1967), Peimbert \& Costero (1969), and Peimbert et al. (2004), we computed the abundances of the chemical elements in the presence of temperature fluctuations. In Table 15 we show the total abundances of $\mathrm{He}, \mathrm{C}, \mathrm{N}, \mathrm{O}, \mathrm{Ne}, \mathrm{S}, \mathrm{Cl}$, and $\mathrm{Ar}$ for $t^{2}>0.00$.

\section{Final discussion}

In this section we discuss additional implications of our results. In particular, we explore possible dependencies of several

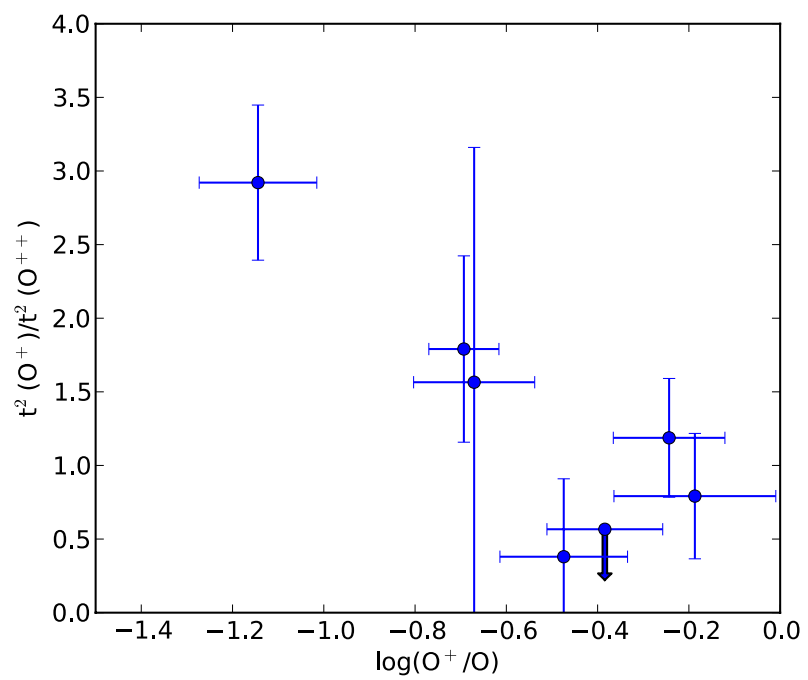

Fig. 16. $t^{2}\left(\mathrm{O}^{+}\right) / t^{2}\left(\mathrm{O}^{++}\right)$ratio vs. the logarithm of the fraction $\mathrm{O}^{+} / \mathrm{O}$.

parameters, such as $\mathrm{ADF}, \mathrm{C} / \mathrm{O}$, and $\mathrm{N} / \mathrm{O}$ on the central star spectral type, and we also compare the Galactic gradient of the $\mathrm{O}$ abundance obtained from our data using ORLs with that obtained from $\mathrm{H}$ II regions.

\subsection{ADF and the abundance ratios versus the spectral type}

Our objects include central stars of spectral types from [WC5] to [WO1], that is, they are among the hottest [WC] stars, all of them are early-[WC], with stellar temperatures from $66 \mathrm{kK}$ to more than $100 \mathrm{kK}$. There are also two wels among our objects (M 1-30 and M 1-61). We recall that we worked with a very biased sample and we showed that this sample has several particularities, for instance, the nebulae are more $\mathrm{N}$ - and $\mathrm{C}$-rich objects than the average of more extended samples. We have searched for some particular behaviour of abundances, that might be correlated with the spectral type. In Fig. 17 upper and middel panels, the $\mathrm{C} / \mathrm{O}$ and N/O abundance ratios (which, as we demonstrated in Sect. 4, are related to the initial stellar mass) are plotted as a function of the spectral type. No correlation is found. C-rich nebulae $(\mathrm{C} / \mathrm{O}>$ 1) are found around stars with types from [WC4] to [WO1] and the same occurs for the O-rich objects $(\mathrm{C} / \mathrm{O}<1)$. That is, objects with a particular spectral type can have any $\mathrm{C} / \mathrm{O}$ ratio. The $\mathrm{N} / \mathrm{O}$ ratio is easier to analyse (Fig. 17 middle panel), because this ratio has a more direct relation with the initial stellar mass (see Fig. 6). The objects with $\log \mathrm{N} / \mathrm{O} \lesssim-0.3$ had initial mass $<4 M_{\odot}$ and spectral types from [WC5.5] to [WO2], and objects with $\mathrm{N} / \mathrm{O} \gtrsim-0.3$ and initial masses larger than $4 M_{\odot}$ also display all [WC] types. In conclusion, the central stars that currently show early-[WC] spectra and that lost their H-rich atmosphere had any initial mass from 1.5 to more than $5 M_{\odot}$. Therefore the [WC] stage is independent of the initial stellar mass, and stars with very different initial masses can pass through the same [WC] stage. A similar conclusion was reported by Peña et al. (2001).

Of particular interest for us is the behaviour of the ADF with the spectral type. From the bottom panel of Fig. 17 we can see that no relation is found, hence, the spectral type and, therefore, the temperature and the wind of the central star are probably not related to the ADF physical origin. 
Table 14. Determinations of the $t^{2}$ parameter.

\begin{tabular}{|c|c|c|c|c|c|c|}
\hline & Cn $1-5$ & $\mathrm{Hb} 4$ & He 2-86 & M 1-25 & M 1-30 & M 1-32 \\
\hline$t^{2}\left(\mathrm{O}^{+}\right)$ & $0.077_{-0.031}^{+0.018}$ & - & $0.111_{-0.020}^{+0.015}$ & $<0.017$ : & $0.038_{-0.013}^{+0.011}$ & $0.057_{-0.035}^{+0.024}$ \\
\hline$t^{2}\left(\mathrm{O}^{++}\right)$ & $0.043 \pm 0.006$ & $0.088 \pm 0.005$ & $0.038 \pm 0.003$ & $0.030 \pm 0.005$ & $0.032 \pm 0.004$ & $0.072 \pm 0.008$ \\
\hline$t^{2}\left(\mathrm{C}^{++}\right)$ & 0.028: & - & - & - & - & - \\
\hline$t^{2}\left(\mathrm{Ne}^{++}\right)$ & 0.002: & 0.058: & 0.010: & 0.076: & $0.026:$ & - \\
\hline$t^{2}\left(\mathrm{He}^{+}\right)$ & $0.042 \pm 0.014$ & $0.082 \pm 0.029$ & $0.036 \pm 0.014$ & $0.035 \pm 0.019$ & $0.013_{-0.013}^{+0.018}$ & $0.038 \pm 0.024$ \\
\hline \multirow[t]{2}{*}{$t^{2}$ (adopted) } & $0.045 \pm 0.006$ & $0.088 \pm 0.005$ & $0.037 \pm 0.003$ & $0.031 \pm 0.005$ & $0.032 \pm 0.004$ & $0.068 \pm 0.008$ \\
\hline & M 1-61 & M3-15 & NGC 5189 & NGC 6369 & PC 14 & Pe 1-1 \\
\hline$t^{2}\left(\mathrm{O}^{+}\right)$ & - & - & $0.019_{-0.019}^{+0.036}$ & - & - & 0.072: \\
\hline$t^{2}\left(\mathrm{O}^{++}\right)$ & $0.033 \pm 0.008$ & $0.048 \pm 0.008$ & $0.050 \pm 0.013$ & $0.034 \pm 0.013$ & $0.047 \pm 0.007$ & $0.046 \pm 0.009$ \\
\hline$t^{2}\left(\mathrm{C}^{++}\right)$ & - & - & $0.075:$ & - & - & - \\
\hline$t^{2}\left(\mathrm{Ne}^{++}\right)$ & $<0.00$ & 0.068: & 0.021: & - & 0.010: & $<0.00$ \\
\hline$t^{2}\left(\mathrm{He}^{+}\right)$ & $0.034 \pm 0.007$ & $0.078 \pm 0.030$ & $0.054 \pm 0.008$ & $0.053 \pm 0.024$ & $0.040 \pm 0.004$ & $0.044 \pm 0.007$ \\
\hline$t^{2}$ (adopted) & $0.034 \pm 0.005$ & $0.050 \pm 0.008$ & $0.051 \pm 0.008$ & $0.038 \pm 0.011$ & $0.042 \pm 0.004$ & $0.045 \pm 0.006$ \\
\hline
\end{tabular}

Table 15. Total abundances for $t^{2}>0.00$.

\begin{tabular}{lccccccc}
\hline \hline & \multicolumn{7}{c}{$12+\log (\mathrm{X} / \mathrm{H})$} \\
\hline Element & $\mathrm{Cn} 1-5$ & $\mathrm{Hb} 4$ & $\mathrm{He} 2-86$ & $\mathrm{M} 1-25$ & $\mathrm{M} 1-30$ & $\mathrm{M} 1-32$ & M1-61 \\
\hline$t^{2}$ & $0.045 \pm 0.006$ & $0.091 \pm 0.005$ & $0.037 \pm 0.003$ & $0.028 \pm 0.005$ & $0.032 \pm 0.004$ & $0.065 \pm 0.008$ & $0.035 \pm 0.005$ \\
\hline $\mathrm{He}$ & $11.19 \pm 0.01$ & $11.06 \pm 0.02$ & $11.09 \pm 0.01$ & $11.09 \pm 0.01$ & $11.15 \pm 0.01$ & $11.10 \pm 0.02$ & $11.04 \pm 0.01$ \\
$\mathrm{C}^{b}$ & $9.18 \pm 0.05$ & $9.07 \pm 0.08$ & $8.87 \pm 0.07$ & $8.93 \pm 0.08$ & $9.30 \pm 0.08$ & $9.75 \pm 0.10$ & $8.67 \pm 0.08$ \\
$\mathrm{~N}$ & $9.00 \pm 0.08$ & $9.05 \pm 0.12$ & $8.90 \pm 0.12$ & $8.61 \pm 0.11$ & $8.87 \pm 0.11$ & $8.79 \pm 0.16$ & $8.47 \pm 0.13$ \\
$\mathrm{O}$ & $9.08 \pm 0.04$ & $9.27 \pm 0.06$ & $9.05 \pm 0.05$ & $9.09 \pm 0.05$ & $9.20 \pm 0.06$ & $9.12 \pm 0.08$ & $8.87 \pm 0.05$ \\
$\mathrm{O}^{c}$ & $9.09 \pm 0.05$ & $9.27 \pm 0.04$ & $9.08 \pm 0.02$ & $9.05 \pm 0.05$ & $9.23 \pm 0.05$ & $9.10 \pm 0.08$ & $8.87 \pm 0.04$ \\
$\mathrm{Ne}$ & $8.69 \pm 0.07$ & $8.79 \pm 0.11$ & $8.56 \pm 0.09$ & $7.95 \pm 0.13$ & $8.40 \pm 0.13$ & $8.12 \pm 0.13$ & $8.01 \pm 0.09$ \\
$\mathrm{~S}$ & $7.55 \pm 0.04$ & $7.66 \pm 0.07$ & $7.55 \pm 0.06$ & $7.50 \pm 0.07$ & $7.71 \pm 0.08$ & $7.62 \pm 0.08$ & $7.37 \pm 0.07$ \\
$\mathrm{Cl}$ & $5.80 \pm 0.04$ & $5.87 \pm 0.07$ & $5.67 \pm 0.05$ & $5.73 \pm 0.07$ & $5.97 \pm 0.07$ & $5.84 \pm 0.07$ & $5.35 \pm 0.05$ \\
$\mathrm{Ar}$ & $6.57 \pm 0.11$ & $7.06 \pm 0.05$ & $6.83 \pm 0.14$ & $7.13 \pm 0.11$ & $7.36 \pm 0.13$ & $7.32 \pm 0.19$ & $6.62 \pm 0.06$ \\
$\mathrm{Fe}$ & $5.75 \pm 0.18$ & $5.82 \pm 0.29$ & $6.25 \pm 0.15$ & $6.38 \pm 0.14$ & $6.02 \pm 0.13$ & $7.12 \pm 0.16$ & $5.53 \pm 0.19$ \\
$\mathrm{Ni}$ & $5.63 \pm 0.23$ & - & $5.86 \pm 0.39$ & - & $4.79 \pm 0.20$ & $6.08 \pm 0.20$ & - \\
\hline $\mathrm{Element}$ & $\mathrm{M} 3-15$ & $\mathrm{NGC} 5189$ & $\mathrm{NGC} 6369$ & $\mathrm{PC} 14$ & $\mathrm{Pe} 1-1$ & $\mathrm{~PB} 8^{a}$ & $\mathrm{NGC} 2867^{a}$ \\
\hline$t^{2}$ & $0.048 \pm 0.008$ & $0.055 \pm 0.008$ & $0.038 \pm 0.011$ & $0.041 \pm 0.004$ & $0.045 \pm 0.006$ & $0.033 \pm 0.005$ & $0.052 \pm 0.01$ \\
\hline $\mathrm{He}$ & $11.03 \pm 0.02$ & $11.09 \pm 0.01$ & $11.02 \pm 0.01$ & $11.03 \pm 0.01$ & $11.02 \pm 0.01$ & $11.09 \pm 0.01$ & $11.06 \pm 0.01$ \\
$\mathrm{C}^{b}$ & $8.85 \pm 0.16$ & $9.02 \pm 0.11$ & $9.00 \pm 0.08$ & $9.02 \pm 0.05$ & $9.12 \pm 0.09$ & $8.85 \pm 0.05$ & $9.25 \pm 0.05$ \\
$\mathrm{~N}$ & $8.71 \pm 0.11$ & $8.79 \pm 0.14$ & $8.34 \pm 0.10$ & $8.39 \pm 0.09$ & $8.29 \pm 0.12$ & $8.55 \pm 0.12$ & $8.29 \pm 0.10$ \\
$\mathrm{O}$ & $9.20 \pm 0.09$ & $8.97 \pm 0.07$ & $8.71 \pm 0.07$ & $9.03 \pm 0.04$ & $8.90 \pm 0.06$ & $9.12 \pm 0.07$ & $8.78 \pm 0.06$ \\
$\mathrm{O}^{c}$ & $9.18 \pm 0.08$ & $8.92 \pm 0.07$ & $8.69 \pm 0.06$ & $9.06 \pm 0.04$ & $8.90 \pm 0.05$ & $9.11 \pm 0.02$ & $8.78 \pm 0.03$ \\
$\mathrm{Ne}$ & $8.46 \pm 0.19$ & $8.49 \pm 0.07$ & $8.09 \pm 0.12$ & $8.48 \pm 0.07$ & $8.27 \pm 0.10$ & $8.52 \pm 0.10$ & $8.24 \pm 0.13$ \\
$\mathrm{~S}$ & $7.71 \pm 0.11$ & $7.33 \pm 0.06$ & $7.14 \pm 0.06$ & $7.40 \pm 0.05$ & $7.07 \pm 0.06$ & $7.79 \pm 0.10$ & $6.92 \pm 0.06$ \\
$\mathrm{Cl}$ & $5.66 \pm 0.09$ & $5.74 \pm 0.04$ & $5.20 \pm 0.06$ & $5.51 \pm 0.05$ & $5.34 \pm 0.07$ & $5.69 \pm 0.10$ & $5.31 \pm 0.06$ \\
$\mathrm{Ar}$ & $6.83 \pm 0.09$ & $6.89 \pm 0.07$ & $6.40 \pm 0.06$ & $6.62 \pm 0.04$ & $6.61 \pm 0.08$ & $6.90 \pm 0.08$ & $6.35 \pm 0.07$ \\
$\mathrm{Fe}$ & $5.92 \pm 0.26$ & $5.04 \pm 0.23$ & - & $5.43 \pm 0.14$ & $5.79 \pm 0.19$ & - & - \\
$\mathrm{Ni}$ & - & - & - & - & & - & - \\
\hline & & & & & & & - \\
\end{tabular}

Notes. ${ }^{(a)}$ From García-Rojas et al. (2009). ${ }^{(b)}$ From recombination lines (see text). ${ }^{(c)}$ From recombination lines (see text).

\subsection{Abundance gradients in the Galactic disk}

Even though our sample is biased towards WRPNe with early[WC] central stars and wels, it is tempting to analyse the behaviour of $\mathrm{O}$ abundance of our objects (which according to stellar evolution models is not significantly modified by the central stars at the Galactic metallicity) relative to the position in the Galaxy. Our objects cover a Galactocentric distance from 1 to about $9 \mathrm{kpc}$. Distances were obtained from
Stanghellini \& Haywood (2010). In Fig. 18 we present the $\mathrm{O} / \mathrm{H}$ abundance ratio (as derived from ORLs) as a function of the Galactocentric distance for our objects. Values for H II regions, also derived from ORLs (Esteban et al. 2005, 2013) and corrected for the $\mathrm{O}$ depleted in dust ( 0.08 dex was added, following Esteban et al. 1998; Mesa-Delgado et al. 2009a) are included. The solar position is shown as well. Clear gradients are found in both cases, although the dispersion is much larger for $\mathrm{PNe}$ than for $\mathrm{H}$ II regions. This large dispersion in $\mathrm{O}$ abundances 

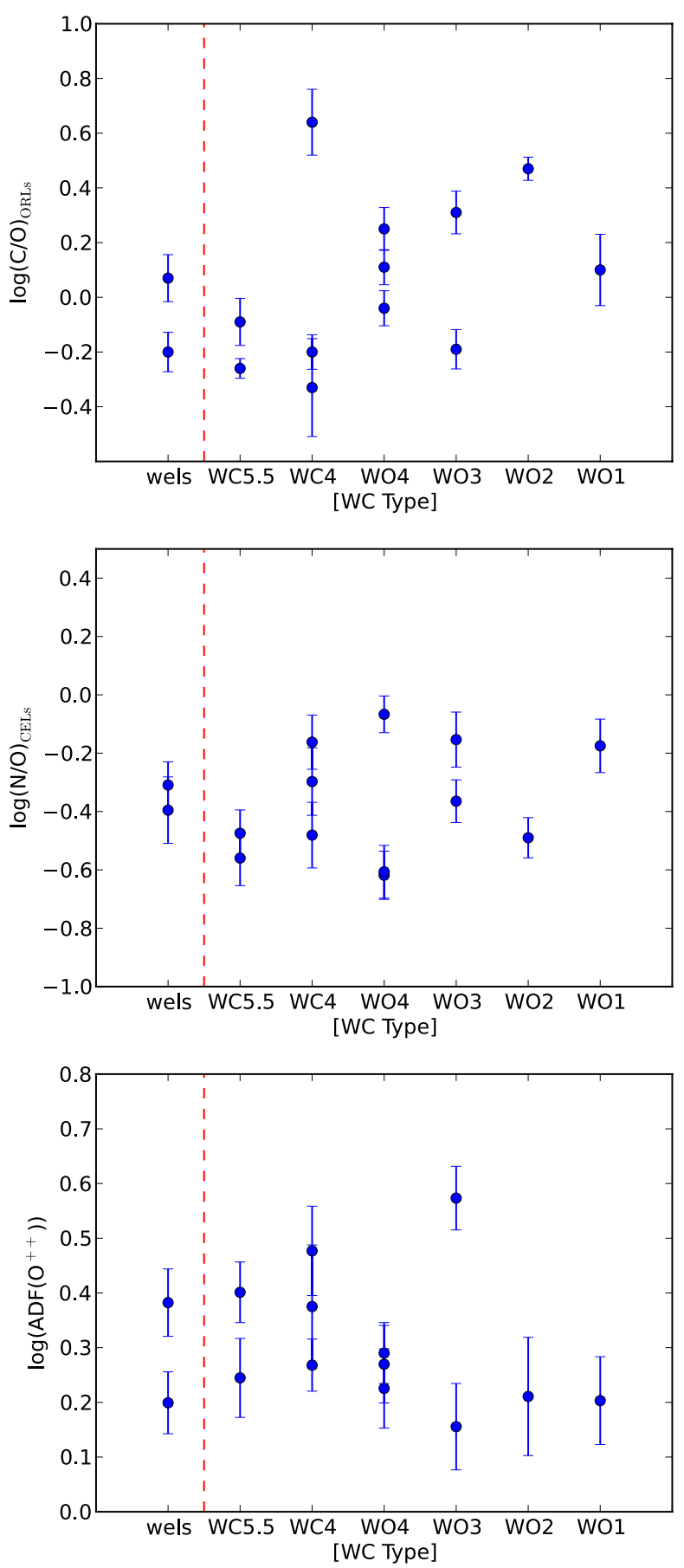

Fig. 17. $\mathrm{C} / \mathrm{O}, \mathrm{N} / \mathrm{O}$, and $\mathrm{ADF}$ vs. central star $[\mathrm{WC}]$ type. The abscense of correlations is discussed in the text.

for PNe at the same Galactocentric distance has been found in all spiral galaxies analysed so far by different authors (e.g., Bresolin et al. 2010; Kwitter et al. 2012; Stasińska et al. 2013) and it is only poorly understood. It is probably due to a combination of effects: uncertainties in distances (in the Milky Way uncertainties are much larger in $\mathrm{PNe}$ than in $\mathrm{H}$ II regions), migration of $\mathrm{PNe}$ that would be at a Galactocentric distance different from their initial position and, very probably, contamination of nebular $\mathrm{O}$ due to stellar nucleosynthesis. A very interesting fact in Fig. 18 is that the $\mathrm{O}$ abundance gradient given by $\mathrm{PNe}$ is flatter than the one derived from $\mathrm{H}$ II regions. Not only that, but it is evident that at the solar distance, the $\mathrm{O}$ abundance is larger for $\mathrm{PNe}$ than for $\mathrm{H}$ II regions (gas+dust) and larger than the solar value

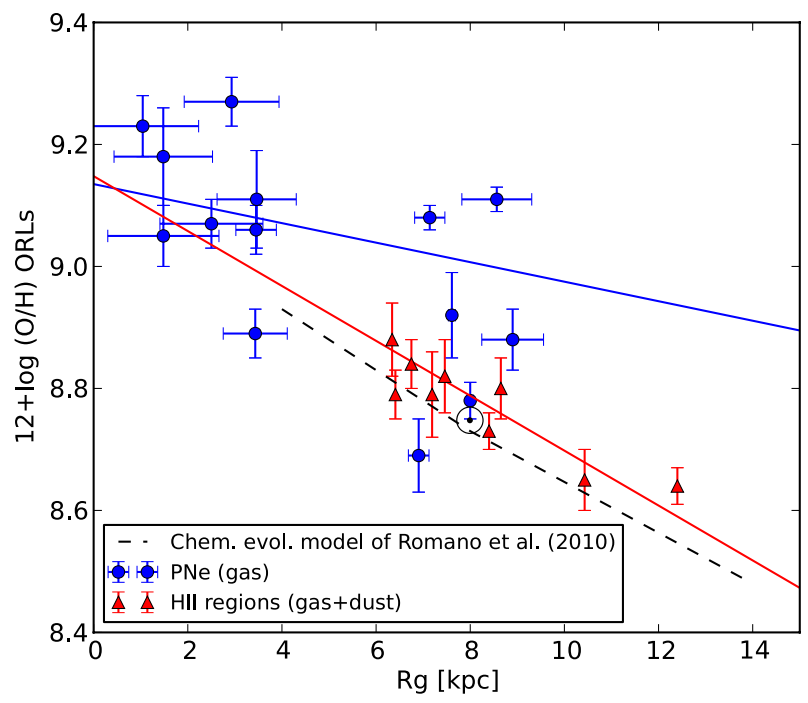

Fig. 18. Oxygen gradient obtained from ORLs for our sample of PNe (blue dots) and from deep spectrophotometric data of $\mathrm{H}$ II regions (red triangles, data taken from Esteban et al. 2005, 2013). Lines are leastsquares fits to the data. The black dashed line represents the results of a chemical evolution model by Romano et al. (2010). Clearly, for PNe, the $\mathrm{O}$ gradient is flatter and, at the solar Galactocentric distance the $\mathrm{O}$ abundance is larger for $\mathrm{PNe}$ than for $\mathrm{H}$ II regions (see text).

(if we were to use abundances from CELs, the same behaviour would be found). Similar results were found by Rodríguez \& Delgado-Inglada (2011) for some PNe and H II regions in the solar vicinity. In addition, this also occurs in other spiral galaxies such as NGC 300 (Stasińska et al. 2013) and M 31 (Zurita \& Bresolin 2012), where external PNe show a larger O abundance than $\mathrm{H}$ II regions in the same zone. It seems most probable that $\mathrm{PNe}$ at these large Galactocentric distances, because they have low metallicity, could be dredging-up $\mathrm{O}$ from their nuclei, therefore $\mathrm{O}$ would not be a suitable element for analysing abundance gradients from $\mathrm{PNe}$.

In Fig. 18 we show the prediction of one of the chemical evolution models presented by Romano et al. (2010) to compare its behaviour with our gradients (black dashed line). From their Fig. $17 \mathrm{~b}$ we chose their model 5, which best reproduces the observations presented there (other models shown in the same figure show a similar behaviour). The main characteristics of model 5 are listed in Table 2 of Romano et al. (2010). We found in the comparison that the gradient predicted in model 5 coincides very well with the gradient from H II regions (chemical evolution models from other authors produce the same result, see Carigi et al. 2005), but it does not reproduces the gradient from $\mathrm{PNe}$ at all. This corroborates that $\mathrm{O}$ in $\mathrm{PNe}$ is affected by other phenomena than those we described above.

\section{Conclusions}

This paper is the third in a series devoted to studying physical conditions and chemical abundances in PNe with [WC] and wel central stars. In García-Rojas et al. (2009) we presented the complete study from deep high-resolution spectrophotometric data obtained at LCO with the 6.5-m Magellan telescope and the spectrograph MIKE for three PNe with [WC] central stars. In García-Rojas et al. (2012) (Paper I) we presented the spectrophotometric data for 12 additional PNe with [WC] and wel central stars obtained with the same configuration. Given the large amount of data, only the physical conditions $\left(T_{\mathrm{e}}\right.$ and $\left.n_{\mathrm{e}}\right)$ 
were discussed in that paper. In this paper we presented the complete chemical abundance analysis.

The ionic chemical abundances were derived using the intensity of collisionally excited lines (CELs) for a large number of ions of different elements. In addition, we determined for the first time in all these objects the $\mathrm{O}^{++}$and $\mathrm{C}^{++}$abundances from optical recombination lines (ORLs). For several objects we also derived for the first time for all of them the ORL abundances of $\mathrm{O}^{+}, \mathrm{C}^{+3}$, and $\mathrm{Ne}^{++}$. We carefully analysed the possible phenomena (such as fluorescence) affecting the recombination line intensities.

From the comparison between CEL and ORL abundances, we derived $\operatorname{ADFs}\left(\mathrm{O}^{++}\right)$between 1.2 and 4, which are in the range of typical ADFs observed in $\mathrm{PNe}$, far from the high values found in other PNe (such as the born-again PNe). Therefore we concluded that the [WC] nature of the central stars does not significantly affect the ADFs. We concluded that the $\mathrm{O}^{++} / \mathrm{H}^{+}$abundance ratio obtained from multiplet 1 is representative of the recombination line abundance, because it is basically the same as that obtained from other multiplets that are excited mainly by recombination $(2,10,20$ and $3 \mathrm{~d}-4 \mathrm{f}$ transitions), especially for objects with highest-quality spectra.

Total abundances were computed by using a carefully chosen set of ICFs. Total abundances for $\mathrm{He}, \mathrm{C}, \mathrm{N}, \mathrm{O}, \mathrm{Ne}, \mathrm{S}, \mathrm{Cl}$, $\mathrm{Ar}, \mathrm{Mg}, \mathrm{Fe}$, and Ni were computed. N/O and $\alpha$-element/O abundance ratios were computed from CELs, while $\mathrm{C} / \mathrm{O}$ ratios were computed from ORLs. We found that our objects are, on average, richer in $\mathrm{N}$ and $\mathrm{C}$ than the average of more extended samples. However, there is no correlation between the nebular chemical abundances and the [WC] stellar type, showing that the [WR] phenomenon can occur in stars of very different stellar masses. By comparing the observed $\mathrm{N} / \mathrm{O}$ and $\mathrm{C} / \mathrm{O}$ abundance ratios with those predicted by stellar evolution models, we estimated that about half of our PNe had progenitors with initial masses similar to or larger than $4 M_{\odot}$. This result is consistent with results from kinematical data, which show that WRPNe are located in a disk thinner than that of average PNe and that hence they are younger and probably more massive objects.

Assuming that the ADF and temperature fluctuations are related phenomena, we estimated the temperature fluctuations parameter, $t^{2}$ from two different methods: 1) applying a chisquared method that minimizes the dispersion of $\mathrm{He}^{+} / \mathrm{H}^{+}$ratios from individual lines; and 2) by comparing the $\mathrm{O}^{++}, \mathrm{O}^{+}, \mathrm{C}^{++}$ and/or $\mathrm{Ne}^{++}$ionic abundances derived from ORLs with those derived from CELs. All methods provided $t^{2}$ values that were consistent within the uncertainties. However, some differences were found for some objects between $t^{2}\left(\mathrm{O}^{+}\right)$and $t^{2}\left(\mathrm{O}^{++}\right)$. The adopted average value of $t^{2}$ was used to correct for the ionic abundances derived from CELs.

In our sample, limited to normal values of ADFs, we found no correlations of ADFs with nebular properties such as metallicity, mean surface brightness, diameter, excitation class, or density, in contradiction with correlations found by other authors for samples with very large ADFs.

The PNe oxygen abundances derived from ORLs are larger than the values from the Sun and Orion nebula. This might be partially explained by the heavy-element fractionation in the Sun since it was formed, but this effect only explains a small percentage of the observed difference. Additionally, chemical evolution models cannot explain the large differences found between $\mathrm{PNe}$ and Orion abundances from ORLs. This is an unresolved question that needs to be analysed in more detail.

Our well-determined PN abundances allowed us to analyse the $\mathrm{O}$ abundance gradient in the Galaxy (Galactocentric distances from 1 to $9 \mathrm{Kpc}$ ). Comparing this with the gradient determined from $\mathrm{H}$ II regions, we found that $\mathrm{PNe}$ gradient is highly dispersed (dispersion range of $\mathrm{O}$ values at the same Galactocentric distance) and is much flatter. At the solar distance, the $\mathrm{O}$ abundances of $\mathrm{PNe}$ are larger than the values for $\mathrm{H}$ II regions. This fact, which is similar to what was found in other spiral galaxies, is not understood so far. Phenomena such as migration or O-enrichment of the nebula due to stellar nucleosynthesis may be playing a role in this question.

By comparing our gradients with predictions from Galactic chemical evolution models, we found that these predictions coincide very well with gradients from $\mathrm{H}$ II regions, but do not reproduce the behaviour of $\mathrm{PNe}$.

Acknowledgements. This work received financial support from the Spanish Ministerio de Educación y Ciencia (MEC) under projects AYA2007-63030 and AYA2011-22614, from CONACYT-México under grants \#43121 and CB2010/153985 and from DGAPA-UNAM, México under grants IN112708 and IN105511. A.M.D. acknowledges support from Comité Mixto ESO-Chile and Basal-CATA (PFB-06/2007) grant. M.T.R. received partial support from PB06 CATA (CONICYT). J.G.R. acknowledges all staff, employees and guests of the Instituto de Astronomía at UNAM, where part of this work was done. C.M. received financial support for his sabbatical at the IAC from the Spanish MEC. We wish to thank the anonymous referee for his/her comments that helped to improve this paper. We also thank L. Carigi, C. Esteban, V. Luridiana, D. A. García-Hernández, A. Peimbert, M. Peimbert, M. Rodríguez, and G. Stasińska for fruitful discussions.

\section{References}

Acker, A., Marcout, J., Ochsenbein, F., Stenholm, B., \& Tylenda, R. 1992, The Strasbourg - ESO catalogue of Galactic planetary nebulae

Asplund, M., Grevesse, N., Sauval, A. J., \& Scott, P. 2009, ARA\&A, 47, 481

Bautista, M. A. 2001, A\&A, 365, 268

Bautista, M. A., \& Pradhan, A. K. 1998, ApJ, 492, 650

Becker, S. R., Butler, K., \& Zeippen, C. J. 1989, A\&A, 221, 375

Benjamin, R. A., Skillman, E. D., \& Smits, D. P. 2002, ApJ, 569, 288

Blöcker, T. 2001, Ap\&SS, 275, 1

Bresolin, F., Stasińska, G., Vílchez, J. M., Simon, J. D., \& Rosolowsky, E. 2010, MNRAS, 404, 1679

Carigi, L., Peimbert, M., Esteban, C., \& García-Rojas, J. 2005, ApJ, 623, 213

Cohen, M., \& Barlow, M. J. 2005, MNRAS, 362, 1199

Delgado-Inglada, G., Rodríguez, M., Mampaso, A., \& Viironen, K. 2009, ApJ, 694,1335

Dopita, M. A., \& Meatheringham, S. J. 1990, ApJ, 357, 140

Escalante, V., \& Morisset, C. 2005, MNRAS, 361, 813

Escalante, V., \& Victor, G. A. 1992, Planet. Space Sci., 40, 1705

Escalante, V., Morisset, C., \& Georgiev, L. 2012, MNRAS, 426, 2318

Esteban, C. 2002, Rev. Mex. Astron. Astrofis. Conf. Ser., 12, 56

Esteban, C., Peimbert, M., Torres-Peimbert, S., \& Escalante, V. 1998, MNRAS, 295,401

Esteban, C., Peimbert, M., García-Rojas, J., et al. 2004, MNRAS, 355, 229

Esteban, C., García-Rojas, J., Peimbert, M., et al. 2005, ApJ, 618, L95

Esteban, C., Bresolin, F., Peimbert, M., et al. 2009, ApJ, 700, 654

Esteban, C., Carigi, L., Copetti, M. V. F., et al. 2013, MNRAS, 433, 382

Fang, X., \& Liu, X.-W. 2013, MNRAS, 429, 2791

Froese Fischer, C., \& Rubin, R. H. 1998, J. Phys. B, 31, 1651

Galavis, M. E., Mendoza, C., \& Zeippen, C. J. 1997, A\&AS, 123, 159

García-Rojas, J., \& Esteban, C. 2007, ApJ, 670, 457

García-Rojas, J., Peña, M., \& Peimbert, A. 2009, A\&A, 496, 139

García-Rojas, J., Peña, M., Morisset, C., Mesa-Delgado, A., \& Ruiz, M. T. 2012, A\&A, 538, A54 (Paper I)

Garnett, D. R., \& Dinerstein, H. L. 2001, ApJ, 558, 145

Garstang, R. H. 1958, MNRAS, 118, 572

Girard, P., Köppen, J., \& Acker, A. 2007, A\&A, 463, 265

Górny, S. K., Stasińska, G., Escudero, A. V., \& Costa, R. D. D. 2004, A\&A, 427, 231

Grandi, S. A. 1976, ApJ, 206, 658

Guzmán-Ramírez, L., Zijlstra, A. A., Níchuimín, R., et al. 2011, MNRAS, 414, 1667

Henney, W. J., \& Stasińska, G. 2010, ApJ, 711, 881

Henry, R. B. C. 1990, ApJ, 356, 229

Henry, R. B. C., Kwitter, K. B., \& Balick, B. 2004, AJ, 127, 2284

Henry, R. B. C., Speck, A., Karakas, A. I., Ferland, G. J., \& Maguire, M. 2012, ApJ, 749, 61 
Herwig, F. 2001, Ap\&SS, 275, 15

Johansson, S., Zethson, T., Hartman, H., et al. 2000, A\&A, 361, 977

Karakas, A. I. 2010, MNRAS, 403, 1413

Karakas, A. I., \& Lattanzio, J. C. 2003, Proc. Astron. Soc. Aust., 20, 393

Karakas, A. I., Lugaro, M. A., Wiescher, M., Görres, J., \& Ugalde, C. 2006, ApJ, 643,471

Kingdon, J., \& Ferland, G. J. 1995, ApJ, 442, 714

Kingsburgh, R. L., \& Barlow, M. J. 1994, MNRAS, 271, 257

Kisielius, R., \& Storey, P. J. 2002, A\&A, 387, 1135

Koesterke, L. 2001, Ap\&SS, 275, 41

Kwitter, K. B., \& Henry, R. B. C. 2001, ApJ, 562, 804

Kwitter, K. B., Lehman, E. M. M., Balick, B., \& Henry, R. B. C. 2012, ApJ, 753, 12

Leisy, P., \& Dennefeld, M. 1996, A\&AS, 116, 95

Liu, X.-W., Storey, P. J., Barlow, M. J., \& Clegg, R. E. S. 1995, MNRAS, 272, 369

Liu, X.-W., Storey, P. J., Barlow, M. J., et al. 2000, MNRAS, 312, 585

Liu, X.-W., Luo, S.-G., Barlow, M. J., Danziger, I. J., \& Storey, P. J. 2001, MNRAS, 327, 141

Liu, Y., Liu, X.-W., Barlow, M. J., \& Luo, S.-G. 2004, MNRAS, 353, 1251

Liu, X.-W., Barlow, M. J., Zhang, Y., Bastin, R. J., \& Storey, P. J. 2006, MNRAS, 368, 1959

Lodders, K. 2003, ApJ, 591, 1220

Lodders, K. 2010, in Principles and Perspectives in Cosmochemistry, eds. A. Goswami, \& B. E. Reddy, 379

Lucy, L. B. 1995, A\&A, 294, 555

Luridiana, V., Morisset, C., \& Shaw, R. A. 2012, in IAU Symp., 283, eds. A. Manchadon, D. Stanghellini, L., \& Schönberner, 422

Marigo, P. 2001, A\&A, 370, 194

Marigo, P., Bernard-Salas, J., Pottasch, S. R., Tielens, A. G. G. M., \& Wesselius, P. R. 2003, A\&A, 409, 619

McNabb, I. A., Fang, X., Liu, X.-W., Bastin, R. J., \& Storey, P. J. 2013, MNRAS, 428,3443

Meléndez, M., Bautista, M. A., \& Badnell, N. R. 2007, A\&A, 469, 1203

Mesa-Delgado, A., Esteban, C., García-Rojas, J., et al. 2009a, MNRAS, 395, 855

Mesa-Delgado, A., López-Martín, L., Esteban, C., García-Rojas, J., \& Luridiana, V. 2009b, MNRAS, 394, 693

Mesa-Delgado, A., Núñez-Díaz, M., Esteban, C., et al. 2012, MNRAS, 426, 614

Nicholls, D. C., Dopita, M. A., \& Sutherland, R. S. 2012, ApJ, 752, 148

Núñez-Díaz, M., Mesa-Delgado, A., Esteban, C., et al. 2012, MNRAS, 421, 3399

Peña, M., Stasińska, G., \& Medina, S. 2001, A\&A, 367, 983

Peña, M., Rechy-García, J. S., \& García-Rojas, J. 2013, Rev. Mex. Astron. Astrofis., 49, 87

Peimbert, M. 1967, ApJ, 150, 825

Peimbert, M. 1990, Rep. Prog. Phys., 53, 1559

Peimbert, M., \& Costero, R. 1969, Boletin de los Observatorios Tonantzintla y Tacubaya, 5, 3

Peimbert, A., \& Peimbert, M. 2005, in The Ninth Texas-Mexico Conference on Astrophysics, eds. S. Torres-Peimbert, \& G. MacAlpine, Rev. Mex. Astron. Astrofis. Conf. Ser., 23, 9
Peimbert, M., \& Peimbert, A. 2006, in Planetary Nebulae in our Galaxy and Beyond, eds. M. J. Barlow, \& R. H. Méndez, IAU Symp., 234, 227

Peimbert, M., Torres-Peimbert, S., \& Ruiz, M. T. 1992, Rev. Mex. Astron. Astrofis., 24, 155

Peimbert, M., Peimbert, A., \& Ruiz, M. T. 2000, ApJ, 541, 688

Peimbert, A., Peimbert, M., \& Luridiana, V. 2002, ApJ, 565, 668

Peimbert, M., Peimbert, A., Ruiz, M. T., \& Esteban, C. 2004, ApJS, 150, 431

Peimbert, A., Peimbert, M., \& Ruiz, M. T. 2005, ApJ, 634, 1056

Pequignot, D., Petitjean, P., \& Boisson, C. 1991, A\&A, 251, 680

Perea-Calderón, J. V., García-Hernández, D. A., García-Lario, P., Szczerba, R., \& Bobrowsky, M. 2009, A\&A, 495, L5

Podobedova, L. I., Kelleher, D. E., \& Wiese, W. L. 2009, J. Phys. Chem. Ref. Data, 38, 171

Porter, R. L., Bauman, R. P., Ferland, G. J., \& MacAdam, K. B. 2005, ApJ, 622, L73

Porter, R. L., Ferland, G. J., \& MacAdam, K. B. 2007, ApJ, 657, 327

Quinet, P. 1996, A\&AS, 116, 573

Robertson-Tessi, M., \& Garnett, D. R. 2005, ApJS, 157, 371

Rodríguez, M. 1996, A\&A, 313, L5

Rodríguez, M. 1999, A\&A, 348, 222

Rodríguez, M., \& Delgado-Inglada, G. 2011, ApJ, 733, L50

Rodríguez, M., \& Rubin, R. H. 2005, ApJ, 626, 900

Romano, D., Karakas, A. I., Tosi, M., \& Matteucci, F. 2010, A\&A, 522, A32

Rola, C., \& Stasińska, G. 1994, A\&A, 282, 199

Ruiz, M. T., Peimbert, A., Peimbert, M., \& Esteban, C. 2003, ApJ, 595, 247

Sawey, P. M. J., \& Berrington, K. A. 1993, At. Data Nucl. Data Tables, 55, 81

Shingles, L. J., \& Karakas, A. I. 2013, MNRAS, 431, 2861

Stanghellini, L., \& Haywood, M. 2010, ApJ, 714, 1096

Stanghellini, L., García-Hernández, D. A., García-Lario, P., et al. 2012, ApJ, 753,172

Stasińska, G. 1978, A\&A, 66, 257

Stasińska, G., Tenorio-Tagle, G., Rodríguez, M., \& Henney, W. J. 2007, A\&A, 471, 193

Stasińska, G., Peña, M., Bresolin, F., \& Tsamis, Y. G. 2013, A\&A, 552, A12

Storey, P. J., \& Hummer, D. G. 1995, MNRAS, 272, 41

Torres-Peimbert, S., \& Peimbert, M. 1977, Rev. Mex. Astron. Astrofis., 2, 181

Torres-Peimbert, S., Peimbert, M., \& Daltabuit, E. 1980, ApJ, 238, 133

Tsamis, Y. G., \& Péquignot, D. 2005, MNRAS, 364, 687

Tsamis, Y. G., Barlow, M. J., Liu, X.-W., Danziger, I. J., \& Storey, P. J. 2003, MNRAS, 338, 687

Tsamis, Y. G., Walsh, J. R., Vílchez, J. M., \& Péquignot, D. 2011, MNRAS, 412, 1367

Verner, E. M., Verner, D. A., Baldwin, J. A., Ferland, G. J., \& Martin, P. G. 2000, ApJ, 543, 831

Wang, W., \& Liu, X.-W. 2007, MNRAS, 381, 669

Waters, L. B. F. M., Beintema, D. A., Zijlstra, A. A., et al. 1998, A\&A, 331, L61

Wesson, R., Liu, X.-W., \& Barlow, M. J. 2003, MNRAS, 340, 253

Wesson, R., Barlow, M. J., Liu, X.-W., et al. 2008, MNRAS, 383, 1639

Whittet, D. C. B. 2003, Dust in the galactic environment (Bristol: Institute of Physics)

Zhang, H. 1996, A\&AS, 119, 523

Zhang, H. L., \& Pradhan, A. K. 1997, A\&AS, 126, 373

Zurita, A., \& Bresolin, F. 2012, MNRAS, 427, 1463 